\title{
Barrierefreier Tourismus
}

\author{
Masterarbeit \\ zur Erlangung des akademischen Grades \\ Master of Science \\ an der Karl-Franzens-Universität Graz
}

vorgelegt von

Karin PLATZER, BA

am Institut für Erziehungs- und Bildungswissenschaft

Begutachter: Ao. Univ. -Prof. Dr.phil.Peter Rossmann

Graz, 2019 


\section{Eidesstattliche Erklärung}

Ich, Karin Platzer, erkläre hiermit ehrenwörtlich, dass die vorliegende Masterarbeit selbstständig und ohne fremde Hilfe verfasst wurde. Es wurden keine Quellen herangezogen, die nicht auch angegeben sind. Verwendete Quellen oder wörtlich bzw. sinngemäß übernommenes Gedankengut wurden als solche kenntlich gemacht.

Graz, am 


\section{Danksagung}

An erster Stelle möchte ich Herrn Ao. Univ.-Prof. Dr. phil. Peter Rossmann danken, für sein genaues Feedback, die schnellen Antworten per Mail und die kompetente und fachliche Betreuung bei der Erstellung dieser Arbeit.

Ein besonderer Dank gilt meinen Klientinnen, die mir bei der Erstellung des Fragebogens geholfen haben, indem sie mir von ihren Erfahrungen berichtet haben.

Danke an Ben und Babsi für die Korrektur der Arbeit.

Danke an Matthias, weil er so viele kleine Arbeiten übernommen hat, damit ich Zeit hatte, diese Arbeit zu verfassen.

Danke an Chrissi, Tanja und Alex, weil durch euch der Master viel mehr Spaß gemacht hat und ich Freundinnen dazugewonnen habe.

Danke an die TeilnehmerInnen der Studie für die vielen positiven Rückmeldungen.

Danke Lisa, dass du deine Englisch-Kenntnisse mit mir geteilt hast. 


\section{Kurzzusammenfassung}

Diese empirische Masterarbeit behandelt die Thematik des barrierefreien Tourismus in Österreich. Die Reiseorganisation von ÖsterreicherInnen mit körperlicher Behinderung und deren Probleme auf Reisen werden erhoben und analysiert. Das Augenmerk liegt dabei auf den Bereichen Planung, An- und Abreise, Unterkunft und Freizeitangebot, wobei der Schwerpunkt auf der Problemfrequenz und Problemrelevanz liegt. Des Weiteren wird die Relevanz von Informationen und weitere Planungsoptionen für Reisende mit körperlicher Behinderung analysiert. Um die benötigten Informationen zu erhalten, wurde ein Online-Fragebogen erstellt und an unterschiedliche soziale Organisationen versendet. Es wurde der Fragebogen von Rannegger (2006) verwendet und durch zusätzliche Fragen ergänzt, um die Hypothesen dieser Arbeit zu beantworten. Die Ergebnisse der Befragung von 111 ProbandInnen wurden mit dem Statistikprogramm „IBM SPSS Statistics 25" ausgewertet und mit Varianzanalysen und Chi-Quadrat Tests analysiert. Die Ergebnisse der Arbeit zeigen, dass Problemaufkommen und Problemempfinden am häufigsten mit dem Freizeitangebot in Verbindung stehen. Die relevantesten Informationen der ProbandInnen betreffen jedoch nicht das Freizeitangebot, sondern die An- und Abreise. Die häufigsten Buchungen werden privat durchgeführt und die häufigsten Urlaubsplanungen über Websites getätigt. 


\begin{abstract}
This empirical master thesis discusses the topic of tourism for all in Austria. This work analyses how Austrians with physical disabilities organize their travels and discusses the problems they face during a journey. Thereby, the focus is on organization, transfer, accommodation and activities at the destination. The problems are evaluated with regard to their frequency of occurrence and their relevance. Furthermore, this thesis evaluates the relevance of the provided travel information as well as the options regarding (travel) planning for people with physical disabilities. In order to collect data, an online questionnaire was used and sent to various social organizations. For the online survey the questionnaire by Rannegger (2006) was adapted to meet the needs of the research questions. In total, 111 questionnaires were evaluated with the statistics program "IBM SPSS Statistics 25" and analyzed by means of ANOVA and Chi-Square tests. In regard to the frequency of problems, the results show that most problems occur at the travel destination and concern activities. However, the most significant information provided by the participants relates to the transfer to and from the destination. The results also show that most bookings are made by private persons and most of the planning is conducted via the internet.
\end{abstract}




\section{Inhalt}

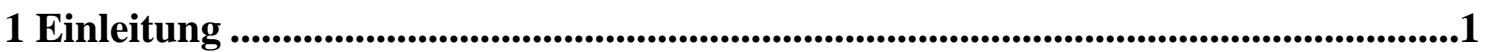

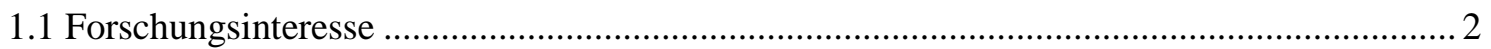

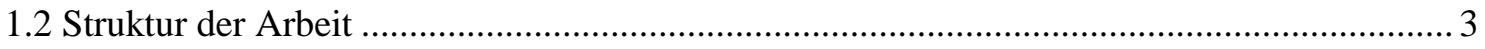

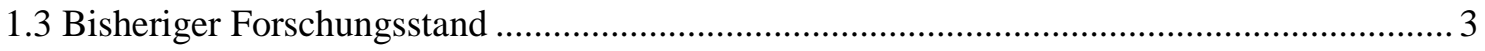

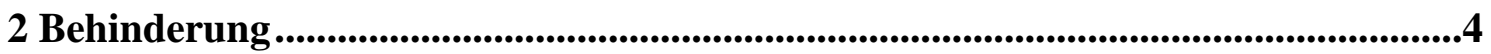

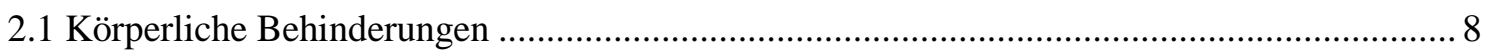

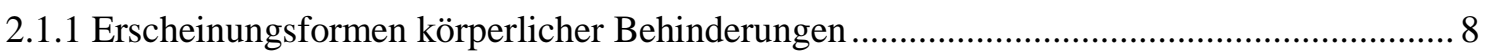

2.2 Soziales und medizinisches Modell von Behinderung im Kontext barrirefreien Reisens .... 12

2.2.1 Soziales Modell und barrierefreier Tourismus ........................................................ 13

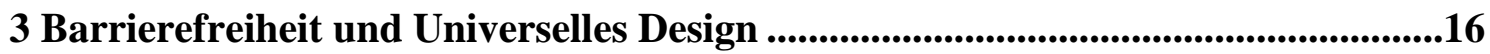

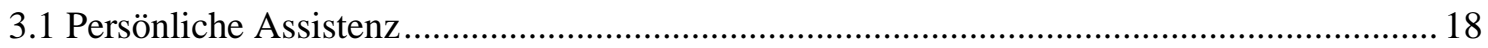

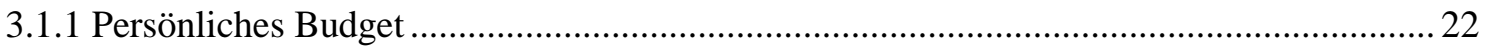

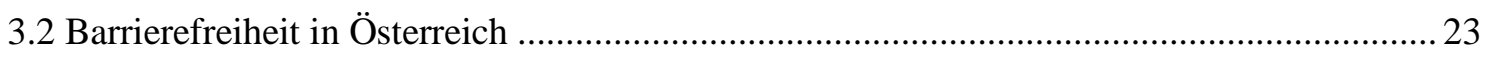

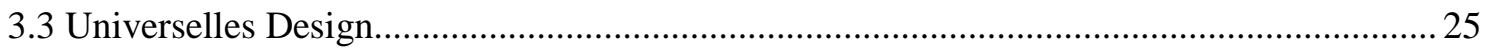

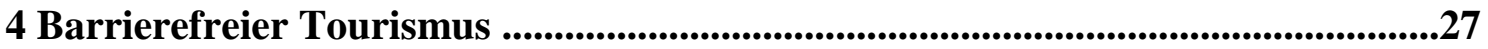

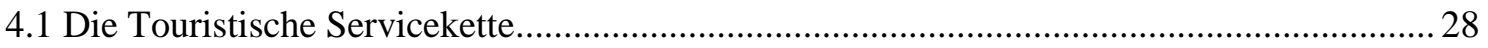

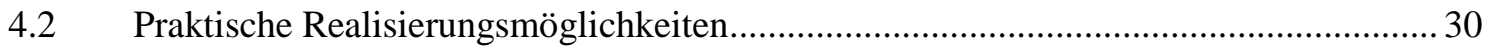

4.2.1 Universelles Design und die touristische Servicekette................................................. 30

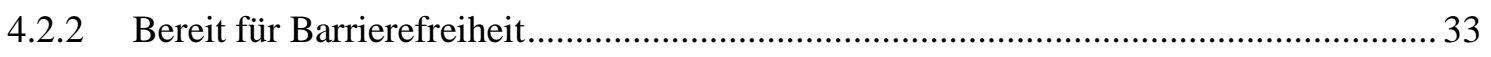

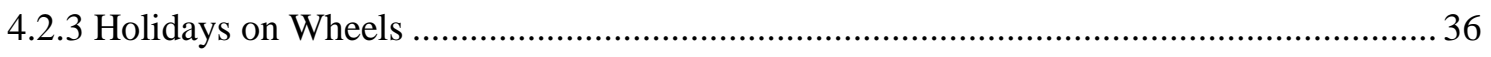

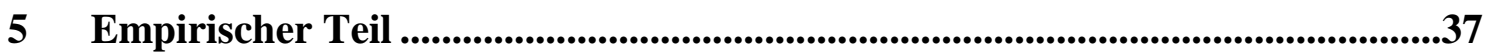

6 Hypothesen und zentrale Fragestellungen ..........................................................37

7 Untersuchungsdesign ......................................................................................41

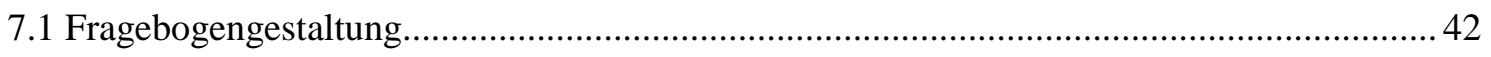

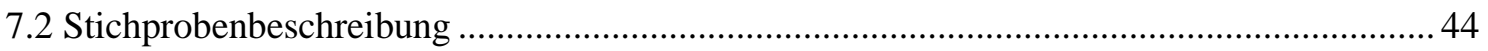


8 Methoden der statistischen Analyse ...........................................................48

$9 \quad$ Ergebnisse der Hypothesentests ....................................................................50

9.1 Welche Barrieren und Grenzen treten am häufigsten im Zuge der touristischen

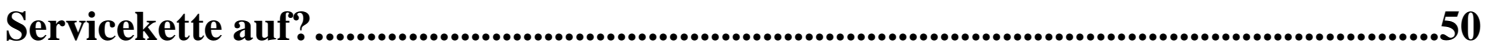

9.1.1 Auftreten von Problemen auf der touristischen Servicekette ............................50

9.1.2 Problemempfinden auf der touristischen Servicekette ..........................................52

9.2 Welche Informationen sind für Reisende mit körperlicher Beeinträchtigung besonders wichtig?....................................................................................................54

9.2.1 Relevanz von Informationen auf der touristischen Servicekette .....................54

9.3 Welche Möglichkeiten zur Realisierung einer Reise werden von Menschen mit

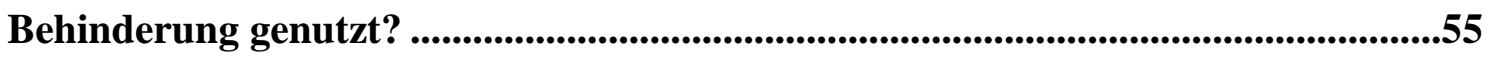

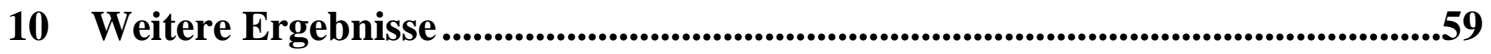

11 Interpretation und Diskussion der Ergebnisse mit Implikationen für touristische Anbieter und Ausblick für weitere Forschung ..........................................61

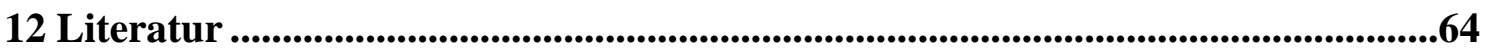

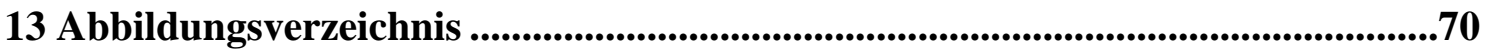

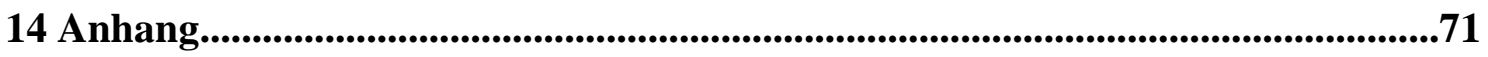

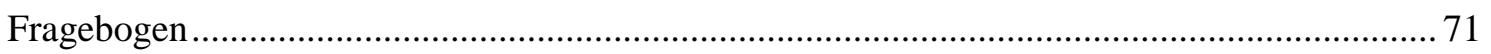

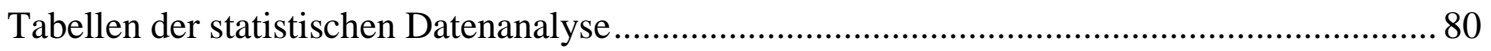

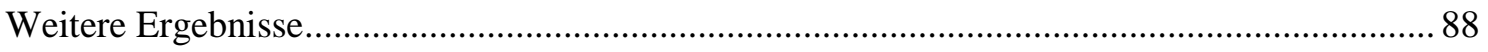




\section{Einleitung}

Aufgrund meiner Arbeit als Reiseassistentin für Menschen mit Behinderung habe ich mich für das Thema der vorliegenden Arbeit entschieden. Die Reisen werden immer sehr gründlich geplant, doch treffe ich immer wieder auf Barrieren, die ein Vorankommen nahezu unmöglich machen. Obwohl es bereits Angebote für barrierefreien Tourismus gibt, stellt sich die Frage, warum eine Reise für Menschen mit Behinderung meist mit erschwerten Bedingungen von statten geht, obwohl im Vorfeld auf Barrierefreiheit geachtet wird. Zimmer, in die kein Rollstuhl passt oder Hotels, in denen es Stufen gibt, sind keine Seltenheit. Auf die Frage, warum dies so sei, wird immer wieder erwähnt, dass das Hotel barrierefrei ist, da es ja auch einen Lift im Gebäude gibt. Durch diese Erfahrungen ist es mir ein großes Anliegen aufzuzeigen, welche Probleme entlang der touristischen Servicekette bei Menschen mit Körperbehinderung auftreten und darauf hinzuweisen, welche Realisierungsmöglichkeiten angeboten und genutzt werden können.

Als Reiseassistentin für Menschen mit Behinderung durfte ich bei einem Netzwerktreffen des Start-Ups „Holidays on Wheels“ einen Vortrag über Barrieren auf Reisen halten und konnte dadurch einen Einblick in deren Arbeit bekommen. Zielgruppenorientiertes Arbeiten zeichnet Holidays on Wheels“، aus. Die Erfahrungen, die ich machen durfte, haben mich sehr motiviert mich näher mit der Thematik auseinanderzusetzen.

Aufgrund meiner Arbeit konnte ich auch bei Vermessungsarbeiten für „Bereit für Barrierefreiheit“ dabei sein. Hierbei hat mich die Genauigkeit und vielseitige Betrachtung von Barrierefreiheit fasziniert, da Menschen mit unterschiedlichen Behinderungen genau sehen können, ob der touristische Betrieb ihren Ansprüchen genügt. Bei der Unterstützung beim Vermessen von Gebäuden durfte ich einer Expertin für Barrierefreiheit behilflich sein und mein Zugang zum Thema wurde so nochmals erweitert. „Holidays on Wheels“ und „Bereit für Barrierefreiheit“" werden in der Arbeit näher vorgestellt.

Die Relevanz dieses Themas zeigt sich durch die hohe Anzahl von Menschen, die auf barrierefreien Tourismus angewiesen sind, wie beispielsweise auch die Anzahl immer älter werdender Menschen. In Österreich geben rund 1,7 Mio. Menschen an, durch eine gesundheitliche Beeinträchtigung eingeschränkt zu sein (Leitner, 2008). 
Trotz dieser hohen Anzahl von Betroffenen entsteht die paradoxe Situation, dass diese Marktlücke im Tourismus nicht geschlossen wird (Wilken, 2016).

In der Arbeit soll beschrieben werden welche Grenzen und Barrieren es für Menschen mit Behinderung auf einer Reise geben kann und wie diese überwunden werden können. Der theoretische Teil klärt Begriffe, die für dieses Thema relevant sind und nimmt Bezug auf bisherige Forschungsergebnisse. Um die Möglichkeit der Realisierung aufzuzeigen, werden Best Practice-Beispiele genannt. Des Weiteren werden organisatorische Anforderungen beschrieben, beispielsweise Reiseassistenz.

Ziel dieser Arbeit ist es, Realisierungsmöglichkeiten und Hindernisse von Reisen für Menschen mit einer körperlichen Beeinträchtigung in Hinblick auf die touristische Servicekette aufzuzeigen. Die Ergebnisse der Forschung sollen dazu beitragen, dass Probleme bei Reisen von Menschen mit Behinderung besser verstanden werden, beispielsweise von touristischen AnbieterInnen. Des Weiteren sollen sie bei der Realisierung von zukünftigen Reisen von Menschen mit körperlichen Beeinträchtigungen behilflich sein.

\subsection{Forschungsinteresse}

- Welche Barrieren und Grenzen treten am häufigsten im Zuge der touristischen Servicekette auf?

- Welche Möglichkeiten zur Realisierung einer Reise werden von Menschen mit Behinderung genutzt?

- Welche Informationen sind für Reisende mit körperlicher Beeinträchtigung besonders wichtig? 


\subsection{Struktur der Arbeit}

Zuerst wird der bisherigere Forschungsstand zu barrierefreien Tourismus in Österreich beschrieben. Im theoretischen Teil werden für die Arbeit relevante Begriffe, wie etwa Behinderung, ausformuliert. Hier wird speziell auf Behinderungen des Bewegungsapparates eingegangen, da Menschen, die davon betroffen sind, die Zielgruppe der empirischen Untersuchung darstellen. Es wird das Soziale und medizinische Modell im Kontext von barrierefreien Reisen beschrieben. Darauf folgt die Beschreibung weiterer relevanter Begriffe wie Barrierefreiheit, Universelles Design, Persönliche Assistenz und die touristische Servicekette. Barrierefreier Tourismus und universelles Design werden durch praktische Beispiele entlang der touristischen Servicekette näher beschrieben.

Der empirische Teil dieser Arbeit befasst sich mit der Untersuchung zu barrierefreiem Tourismus in Österreich. Ziel dieser Studie ist es, einen Überblick über Probleme und Möglichkeiten barrierefreien Reisens zu bieten, um zukünftige Planungen zu erleichtern und Verbesserungsmöglichkeiten für touristische Anbieter aufzuzeigen. Im ersten Abschnitt werden die Forschungsfragen präzisiert und davon ausgehend die zugehörigen Hypothesen beschrieben. Anschließend werden die verwendeten Erhebungsinstrumente vorgestellt. Das Untersuchungsdesign wird mit den Methoden der Fragebogengestaltung, den Methoden der Datenerhebung und dem Stichprobenplan vorgestellt. Daraufhin werden die Ergebnisse der Arbeit angeführt. Im letzten Kapitel werden die Ergebnisse der Hypothesen diskutiert und interpretiert. Dabei werden Lösungsmöglichkeiten angeführt, die zukünftige Barrieren auf Reisen verringern können. Hierfür werden Verbesserungsvorschläge für touristische Anbieter angeführt.

\subsection{Bisheriger Forschungsstand}

Barrierefreier Tourismus ist in Österreich kaum quantitativ erforscht. Die meisten Studien wurden in Deutschland erarbeitet und die Ergebnisse wurden auf den österreichischen Markt umgelegt (Rannegger, 2006). Es werden hauptsächlich qualitative Methoden eingesetzt, wie beispielsweise bei Wrussnig (2010). Die Diplomarbeit von Rannegger (2006) befasst sich mit barrierefreiem Tourismus in Österreich. Es handelt sich dabei um die Analyse des Reiseverhaltens und der dabei auftretenden Probleme entlang der touristischen Servicekette von Menschen im Rollstuhl in Österreich. 
Er wollte die Lücke an quantitativen Daten zu diesem Thema schließen. Es wurden Daten über das Reiseverhalten, die Reisehäufigkeit, die Reiseabsicht und die Gründe einer Reiseabstinenz von RollstuhlfahrerInnen erhoben.

Ein wesentliches Ziel seiner Forschung ist die Auflistung von Reiseproblemen entlang der touristischen Servicekette von Menschen im Rollstuhl aus Österreich. Es wurden österreichweit 316 Menschen im Rollstuhl befragt, davon waren 302 Fragebögen gültig. Die häufigsten Probleme betrafen die Zugänglichkeit aufgrund von baulichen Barrieren allgemein während der gesamten Reise und die sanitären Einrichtungen (Rannegger, 2006).

Eine weitere Quelle von quantitativen Daten aus Österreich bietet die Projektseminararbeit mit dem Thema barrierefreier Tourismus von Flucher, Parigger \& Zirngast (2013), die an der Karl-Franzens-Universität verfasst wurde. Es werden darin die Probleme von Menschen im Rollstuhl entlang der touristischen Servicekette evaluiert.

\section{Behinderung}

2015 gab in der Europäischen Union ein Viertel (25,3 \%) der Bevölkerung ab 16 Jahren an, von Einschränkungen betroffen zu sein, die über sechs Monate anhalten (Eurostat, 2016).

In Österreich wurde von Statistik Austria eine Befragung zum Thema Menschen mit Behinderung im Auftrag des Bundesministeriums für Arbeit, Soziales und Konsumentenschutz durchgeführt. Bei der Befragung gaben 20,5\% an, von einer Dauerbehinderung betroffen zu sein. Das entspricht hochgerechnet ca. 1,7 Millionen Menschen. Körperliche Behinderungen kommen in Österreich am häufigsten vor. Sie betreffen 13 \% der Bevölkerung, das entspricht ca. eine Million Personen (BMASK, 2013). Werden diese Statistiken betrachtet, zeigt sich durch die hohe Anzahl an Menschen mit Behinderung bzw. Beeinträchtigung die große Bedeutung von barrierefreiem Tourismus. 
In der Fachliteratur wird über den Behinderungsbegriff viel diskutiert. Es wird unter anderem von Menschen mit besonderen Bedürfnissen gesprochen. Dieser Begriff wird hier nicht verwendet, da alle Menschen, Menschen mit einer Körperbehinderung eingeschlossen, sowohl individuelle als auch allgemeine Bedürfnisse erfüllen möchten, welche für sie besonders sind. (Hackl, 2014).

In dieser Arbeit wird nur der Begriff der körperlichen Behinderung besprochen. Die Bedeutung des Begriffs Behinderung hat sich über die Zeit verändert. Vor allem in den letzten Jahrzehnten, beginnend 1961, hat sich das Verständnis von Behinderung stark gewandelt. Laut dem deutschen Bundessozialhilfegesetz von 1961 sind Behinderte Personen, die nicht nur vorübergehend körperlich, geistig oder seelisch wesentlich behindert sind. Daraus lässt sich nur schließen, dass Behinderung nicht nur vorübergehend ist und alle Dimensionen menschlichen Verhaltens und Erlebens betreffen kann. 1986 kam es zu einer genaueren Einteilung im Schwerbehindertengesetz. Eine Behinderung liegt erst vor, wenn die betroffene Person über 6 Monate behindert ist. Die Auswirkung der Behinderung wird nun auch gemessen als Grad der Behinderung. Dieser wird in Zehnerschritten angegeben und kann bis 100 reichen (Leyendecker, 2005).

In den 90er Jahren des 20. Jahrhunderts wurde der soziale Kontext von Behinderung mit in Betracht gezogen. Es wurde ergänzt, dass Behinderung erst unter sozialem Bezug in Erscheinung tritt. Hier beschreibt Schellhorn: „,körperlich wesentliche Behinderte... sind Personen, bei denen infolge einer körperlichen Regelwidrigkeit die Fähigkeit zur Eingliederung in die Gesellschaft in erheblichem Umfange beeinträchtigt ist“" (Schellhorn, 1996 zit. n. Leyendecker, 2005).

Die Begriffe Behinderung und Schädigung sind zu unterscheiden, da der soziale Kontext hier relevant ist. Dieser Unterschied kann mithilfe eines Beispiels aufgezeigt werden:

Eine Schülerin mit Querschnittlähmung, die nur die Beine betrifft, sitzt in der Klasse mit anderen SchülerInnen zusammen und sie erledigt eine Schreibarbeit. In dieser Situation ist sie nicht in der erforderlichen Funktion beeinträchtigt oder in der sozialen Teilhabe behindert. Die Schülerin wird erst behindert, wenn alle anderen in der Pause aufspringen und über die Stiege in den Schulhof laufen und die Schülerin nicht folgen kann (Leyendecker, 2005). 
Das Beispiel kann auch bei barrierefreien Reisen angewendet werden. Wenn die gleiche Schülerin eine Reise antritt und auf bauliche Barrieren oder das Fehlen von Rampen trifft wird sie behindert. Bei einem barrierefreien Setting auf der gesamten Reise wäre sie nicht beeinträchtigt.

Um diesen Unterschied zwischen Schädigung und Behinderung miteinzubeziehen, wurde 2001 von der WHO eine begriffliche Differenzierung vorgenommen. In der International Classification of Functioning, Disability and Health (ICF) wird der ganze Lebenshintergrund des Menschen mit Behinderung miteinbezogen. Es wird folgende Unterteilung beschrieben:

- Schädigung (impairment) wird durch die Begriffe Körperfunktionsstörung und Strukturschaden beschrieben.

- Fähigkeitsbeeinträchtigung wird beschrieben als Beeinträchtigung der Aktivität (activity limitation).

- Soziale Benachteiligung wird als Beeinträchtigung der Partizipation beschrieben (participation restriction).

In der ICF werden auch weitere Aspekte in Betracht gezogen, wie die

- äußeren Einflüsse: fördernde oder beeinträchtigende Umweltfaktoren

- innere Einflüsse: diese beinhalten personenbezogene Faktoren.

Eine Behinderung liegt nicht zwingend vor, weil eine Schädigung vorliegt. Eine Behinderung entsteht durch die Einschränkung der Aktivität in Verbindung mit Beeinträchtigung der Partizipation. Die folgende Grafik zeigt die Wechselbeziehung zwischen den unterschiedlichen Komponenten des Behinderungsbegriffs auf (Leyendecker, 2005). 


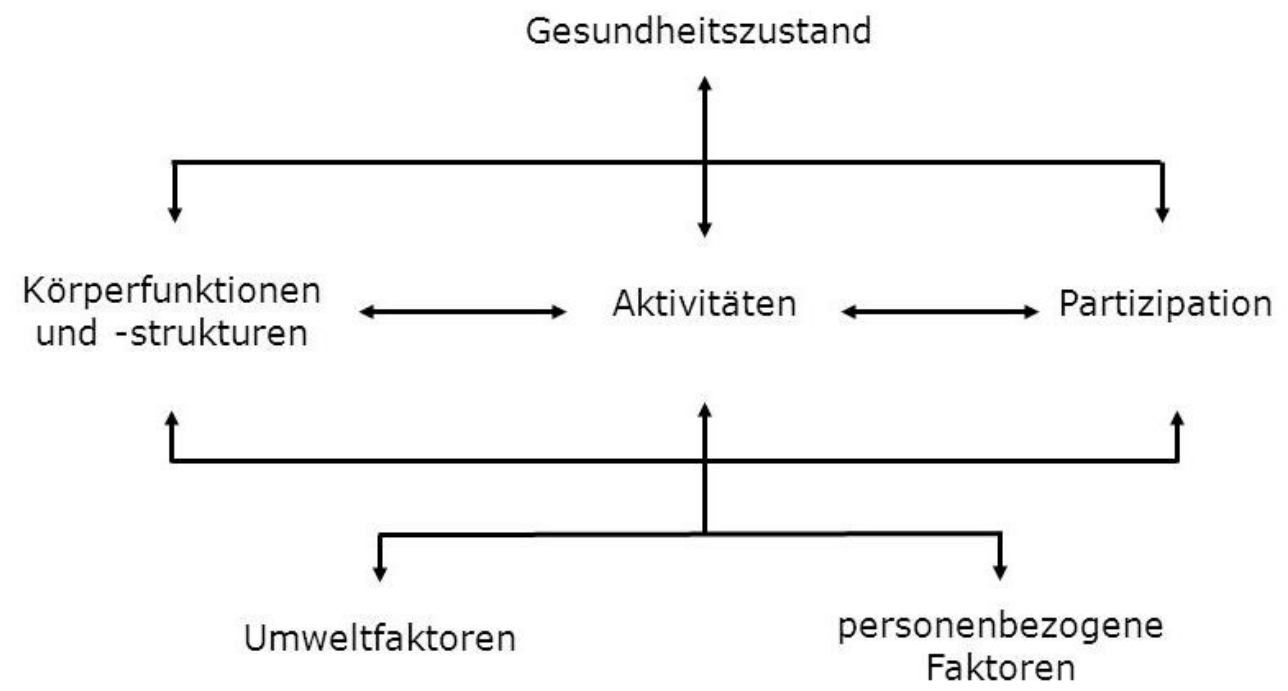

Abbildung 1: Wechselwirkungen zwischen den Komponenten der ICF (DIMDI 2005, 23).

Wie dieser Überblick zeigt, gibt es eine Vielzahl an Definitionen für den Begriff Behinderung. Die World Health Organisation (WHO) bringt noch einen weiteren Aspekt der Begriffsdebatte ein: Manche mögen es lieber, als behinderte Personen bezeichnet zu werden und andere als Menschen mit Behinderung. Die betroffenen Menschen haben ein Recht darauf, so genannt zu werden, wie sie es wünschen (Puschke, 2005).

Neu an der ICF ist die Einbeziehung von Umweltfaktoren wie Assistenz- oder Heilmittelbedarf. Auch persönliche Faktoren wie Geschlechtszugehörigkeit, Alter oder Ethnie sollen hinzugezogen werden. Vereinfacht gesagt wird bei der Ermittlung der ICF ein sehr umfassender Fragenkatalog ausgefüllt. In jeder Kategorie wird eingeteilt, ob z.B. die körperliche, individuelle und gesellschaftliche Behinderung kein Problem, ein geringes, gemäßigtes, schweres oder vollständiges Problem darstellt. Gemessen wird an der Norm von Menschen ohne Behinderung. Diese Tatsache wird von VertreterInnen der Behindertenbewegung kritisiert (Puschke, 2005).

Anhand der aktuellen Anlehnung an die ICF der WHO werden Begriffe wie körperliche, geistige und seelische Beeinträchtigung und solche des Sprach-Hörvermögen als Teil menschlicher Vielfalt verdeutlicht (Wilken, 2015). 
In dieser Arbeit werden ausschließlich Menschen mit körperlicher Behinderung befragt. Wie körperliche Behinderung definiert wird und in welchen Erscheinungsformen diese auftreten kann soll nun dargestellt werden.

\subsection{Körperliche Behinderungen}

Bei der Definition von Körperbehinderung müssen die Komponenten Schädigung, Aktivität und Partizipation berücksichtigt werden. Erst die fehlenden Möglichkeiten der Partizipation, stellt die eigentliche Behinderung dar, wie Leyendecker (2005) beschreibt.

Als körperbehindert wird eine Person bezeichnet, die infolge einer Schädigung des Stütz- und Bewegungssystems, einer anderen organischen Schädigung oder einer chronischen Krankheit so in ihren Verhaltensmöglichkeiten beeinträchtigt ist, dass die Selbstverwirklichung in sozialen Interaktionen erschwert ist. (Leyendecker, 2005, S.21).

Die unterschiedlichen Ausprägungen von Körperbehinderung entstehen aufgrund einer Schädigung einer körperlichen Funktion oder Struktur. Dies beinhaltet Schädigungen des Nervensystems, der Muskulatur, des Knochengerüstes, chronische Krankheiten und organische Fehlfunktionen. Die häufigste Körperbehinderung ist die cerebrale Bewegungsstörung. Diese tritt bei Schädigungen der Muskulatur und des Knochengerüstes sowie bei gewissen chronischen Krankheiten auf (Leyendecker, 2005).

\subsubsection{Erscheinungsformen körperlicher Behinderungen}

Körperliche Behinderungen werden in diesem Kapitel mittels der drei Dimensionen von Behinderung erläutert. Es wird mit der Beschreibung der körperlichen Schädigungen (physical impairments) begonnen.

Am Häufigsten tritt bei Kindern und Jugendlichen die cerebrale Bewegungsstörung mit einer Inzidenzrate von 3:1000 auf. Aufgrund der hohen Anzahl von Betroffenen wird genauer auf diese Schädigung eingegangen. Des Weiteren werden die Spina Bifida mit einer Inzidenzrate von 0,5-1:10000 und Multiple Sklerose beschrieben (Leyendecker, 2005). 


\section{Cerebralparesen}

Die Cerebralparesen verursachen Bewegungsstörungen, die durch Fehlfunktionen oder Lähmungen in Gehirn und Rückenmark ausgelöst werden. Cerebralparesen entstehen meist durch Komplikationen während oder nach der Geburt und damit verbundene Schädigungen des Gehirns. Diese Schädigungen können durch Infektionen, Sauerstoffmangel oder Gehirnblutungen hervorgerufen werden. Da sie meist mit Komplikationen bei der Geburt in Verbindung steht, wird auch von der infantilen Cerebralparese gesprochen. Ein späteres Auftreten von Cerebralparesen, beispielsweise durch ein Schädel-Hirn-Trauma, ist jedoch möglich (Leyendecker, 2005).

Diese Schädigung ist gekennzeichnet durch abnorme Muskelspannungen, gestörte Koordination von Bewegungsabläufen und veränderte Ausdrucksbewegungen. Es werden drei Erscheinungsformen beschrieben.

- Bei der spastischen Erscheinungsform ist die Muskelspannung erhöht, dadurch sind die Bewegungen verkrampft und langsam. Diese Erscheinungsform tritt am Häufigsten auf.

- Die athetotische Erscheinungsform ist von einem schwankenden Muskeltonus geprägt. Bewegungen werden unwillkürlich und ruckartig ausgeführt.

- Bei der ataktischen Parese besteht eine niedrige Muskelspannung und die Betroffenen haben dadurch Unsicherheiten bei Bewegungsabläufen und eine gestörte Gleichgewichtsreaktion (Leyendecker, 2005).

Bei Cerebralparesen sind Grobmotorik und Feinmotorik betroffen. Sie treten häufig in Verbindung mit sensorischen Behinderungen und Störungen der Sprache auf (Leyendecker, 2005).

Cerebralparesen werden nach den betroffenen Körperregionen eingeteilt:

- Tetraplegien: alle 4 Extremitäten sind betroffen

- Diplegie: betrifft Arme oder Beine

- Hemiplegie: eine Körperseite ist betroffen (Gruber \& Ledl, 2004). 


\section{Querschnittlähmungen}

Eine Querschnittlähmung entsteht durch eine Schädigung des Rückenmarks. Je nach der Höhe der Schädigung können alle vier Extremitäten (Tetraplegie) betroffen sein, es kann aber auch eine Paraplegie vorliegen. Spastische und ataktische Lähmungen können die Folge sein. Weiters können querschnittgelähmte Personen auch von Inkontinenz und Störungen der Temperaturregulation betroffen sein (Hülshoff, 2011).

Ursachen einer Querschnittlähmung können Unfallverletzungen, entzündliche Erkrankungen und Tumore sein. Querschnittlähmungen können auch pränatal entstehen. Diese spezielle Form wird Spina Bifida genannt (Leyendecker, 2005).

\section{Spina Bifida}

Spina Bifida ist eine Fehlbildung der Wirbelsäule, bei der das Rückenmark an einer Stelle offen liegt. Nach der Geburt wird diese Fehlbildung operativ geschlossen. Je nach der auftretenden Stelle und der Ausprägung entstehen Lähmungen der Beine und verringerte Kontrollfähigkeit über Blase und Mastdarm (Leyendecker, 2005).

\section{Multiple Sklerose}

Bei der multiplen Sklerose handelt es sich um eine Schädigung der Schicht, die die Nervenfasern umhüllt. Es ist eine entzündliche Erkrankung, die das Gehirn angreift und vernarbtes Bindegewebe umbaut. Daraus folgt eine Störung der neuronalen Leitung. Die Symptome variieren, je nachdem welcher Teil im Gehirn und Rückenmark betroffen ist (Leyendecker, 2005).

Die Ursache der multiplen Sklerose ist nicht eindeutig bekannt. Es wird vermutet, dass genetische Faktoren eine Rolle spielen. Multiple Sklerose gehört zu den Entmarkungserkrankungen, die ganz überwiegend die weiße Substanz des Nervensystems befallen und bei denen es zu einer Schädigung oder Auflösung der Markscheiden kommt. Letztlich wird die multiple Sklerose mittlerweile als eine Autoimmunerkrankung verstanden. Es wird vermutet, dass multiple Sklerose durch Viren aktiviert wird. Dadurch sollen entzündungsspezifische Stoffe die Myelinschicht schädigen (Hülshoff, 2011). 
Cerebralparesen, multiple Sklerose und Querschnittlähmungen gehören zur Gruppe der Schädigungen von Gehirn und Rückenmark. Im nächsten Schritt werden Schädigungen der Muskulatur und des Knochengerüsts angeführt. Es handelt sich bei Schädigungen der Muskulatur und des Knochengerüsts häufig um erblich bedingte Krankheiten. Es bestehen hier unterschiedliche Verläufe und Erscheinungsformen (Leyendecker, 2005).

\section{Muskeldystrophie}

Die Muskeldystrophie kann einen sehr schwerwiegenden Verlauf nehmen. Bei den Betroffenen kann ein Muskelabbau bis zum völligen Verlust der Funktionsfähigkeit fortschreiten. Gutartige Muskeldystrophien, wie beim Typ Becker, beeinflussen die Lebenserwartung kaum. Eine spezielle Form der bösartigen Muskeldystrophie, Typ Duchenne, kann nur auf männliche Nachkommen vererbt werden. Die Auffälligkeiten beginnen zwischen dem 3. Und 5. Lebensjahr. Bereits im Schulalter ist die Gehfähigkeit betroffen. Der Muskelschwund kann bereits im jungen Erwachsenenalter zum Tod führen (Leyendecker, 2005).

Weitere Schädigungen der Muskulatur und des Knochengerüsts werden nun kurz aufgelistet:

- Missbildungen der Gliedmaßen

- Verlust der Gliedmaßen

- Fehlstellungen der Wirbelsäule

- Extreme Wachstumsstörungen

$\mathrm{Zu}$ den Körperschädigungen zählen auch Einschränkungen der Bewegungsfähigkeit, die auf chronische Krankheiten oder auf die Fehlfunktionen von Organen zurückzuführen sind. Diese beinhalten beispielsweise rheumatische Erkrankungen oder verschiedene Hauterkrankungen (Leyendecker, 2005). 
Die Beeinträchtigung der Aktivität (activity limitation) kann auf Schädigungen zurückgeführt werden, da ein Zusammenhang zwischen Bewegung und psychischen Funktionen besteht. Die Bereiche Wahrnehmung, Intelligenz, Lernen und Kommunikation können betroffen sein.

Körperbehinderte Menschen werden häufig über den körperlichen Defekt definiert und nicht über ihre individuelle Persönlichkeit. Durch eine positive Zuschreibung des Lieben und Geduldigen werden viele Menschen mit Behinderung auch stigmatisiert und konformistisch erzogen. Viele wollen diesen Rollenerwartungen nicht entsprechen und wählen den Weg des Außenseiters. Die Selbstfindung von Menschen mit einer körperlichen Behinderung stellt eine Gratwanderung zwischen sozialer Anpassung und Selbstbehauptung dar (Leyendecker, 2005).

Bisher wurde hauptsächlich die medizinische Sichtweise, die sogenannten physical impairments und die activity limitations, beschrieben. Das sozial- und gesellschaftlich bestimmte Phänomen von Behinderung wird im nächsten Kapitel, durch die Beschreibung des sozialen Modells, in den Fokus gerückt.

\subsection{Soziales und medizinisches Modell von Behinderung im Kontext barrire- freien Reisens}

Nach der Mitte des letzten Jahrhunderts begann ein Prozess, in dem das Verständnis von Behinderung einen Wandel vom individuellen medizinischen Problem zum gesamtgesellschaftlichen Phänomen durchlief. Dieser Vorgang wurde von Aktivisten der 1960er Jahre vorangetrieben. Menschen mit körperlichen, sensorischen und kognitiven Behinderungen erlebten eine große Bandbreite an umgebungsbedingten und sozialen Barrieren. Das medizinische Modell beinhaltet die Vorstellung, dass Menschen flexibel sind und sich anpassen können, jedoch die physische und soziale Umgebung nicht. Daher wurden Menschen mit Behinderung wie Objekte behandelt, die medizinisch behandelt, verändert, verbessert und „normal“" gemacht wurden (Barnes, 2011). 
Aus diesen Gründen bildeten sich seit den 1960er Jahren weltweit Organisationen, die sich gegen das diskriminierende medizinische Modell richteten und es entstand das soziale Modell von Behinderung (Barnes, 2011).

Puschke (2005) und Link (2016) beschreiben, wie sich die beiden Modelle unterscheiden. Das medizinische Modell beschreibt Behinderung als persönliches Problem und aus der Schädigung resultieren die Einschränkungen an der Teilhabe am gesellschaftlichen Leben (participation restriction). Das soziale Modell ändert den Blickwinkel. Dieses Modell sieht das Problem in der Umwelt, von welcher der Mensch behindert wird. Nicht der Mensch ist behindert, sondern er wird von seiner Umwelt behindert.

Die WHO wählt einen Weg zwischen den Modellen, da eine Behinderung hier aus den Barrieren in der Umwelt resultiert oder aus einer Schädigung heraus. Die gesellschaftliche Teilhabe und die körperliche Funktionsfähigkeit werden bei der WHO immer an der Norm der Menschen ohne Beeinträchtigung gemessen. Dadurch ergibt sich die Frage danach, was ein richtig funktionierender Körper ist, da viele Personen keine Behinderung hätten, würden sie in einer barrierefreien Umwelt leben. Barrierefreie Reisen könnten auch einfacher durchgeführt werden, wenn die Umwelt barrierefrei gestaltet wäre, wie beispielsweise nach dem Konzept des universellen Designs (Puschke, 2005).

\subsubsection{Soziales Modell und barrierefreier Tourismus}

Barrierefreie Reisen und universelles Design sind Spezialgebiete von Christiane Link. Sie ist eine Vertreterin des sozialen Modells und arbeitet am Projekt „Transport for All“. Sie hielt 2016 einen Vortrag über die negativen Aspekte des medizinischen Modells, wobei ein Teil dieser hier angeführt wird. Sie berichtet von der Problematik, dass Behinderung von Ärzten, Sonderpädagogen, Sozialarbeitern und Physiotherapeuten definiert und bestimmt wird und nicht von behinderten Menschen selbst.

„Hinter dieser rein medizinischen Definition schwingt auch immer die Botschaft mit: Du bist nicht in Ordnung, wie du bist. Niemand hinterfragt, was das eigentlich für behinderte Kinder bedeutet, die von klein auf mit dieser Botschaft aufwachsen: Du bist nicht in Ordnung. Dabei sind sie sehr wohl in Ordnung. Sie müssen nur Dinge anders machen, sie bewegen sich anders fort, sie lesen Lippen und sie brauchen ein barrierefreies Umfeld, Assistenz und bedarfsgerechte Unterstützung“(Link, 2016, S. 1). 
Link (2016) spricht in ihrem Vortrag genau die Kernpunkte von barrierefreiem Tourismus an:

1. Barrierefreies Umfeld

2. Assistenz

3. Bedarfsgerechte Unterstützung

Link ist Journalistin und benötigt einen Rollstuhl. Sie ist viel unterwegs und hat bereits mehr als 1000 Flüge hinter sich, die sie meist allein zurücklegt. Bis heute wird sie noch gefragt, ob ein Beruf, in dem sie viel reist, der geeignete für sie sei. Auch einige Fluggesellschaften bestehen darauf, dass sie bei ihren Reisen eine Begleitung hat (Link, 2016). Hier zeigt sich, dass das medizinische Modell, welches defizitorientiert ist, auch im Bereich des Reisens vertreten ist. Das soziale Modell würde für Rahmenbedingungen sorgen, die eine Teilhabe ermöglichen. Dieses Modell beschäftigt sich mit folgenden Fragen: „Was muss getan werden, um Teilhabe zu ermöglichen?“. „Was kann die Gesellschaft tun?“A Antworten können beispielsweise sein von Beginn an barrierefrei zu planen und zu bauen oder im Bestand umzubauen, oder etwa MitarbeiterInnen entsprechend sozial zu schulen, damit sie Menschen mit Behinderung nicht den Zugang in das Museum oder ins Flugzeug verweigern. Vor allem aber braucht es die Bereitschaft, den Fokus auf das Ermöglichen und nicht auf das Behindern zu legen. Sehr häufig ist es vielmehr eine Frage der Einstellung und nicht so sehr eine Frage des Geldes. Es geht nicht darum, jemandem die optimale medizinische Versorgung abzusprechen, aber, wenn man am körperlichen Zustand nichts ändern kann, wäre es von Vorteil, die Umwelt, die Vorgänge, die Gegebenheiten an behinderte Menschen anzupassen (Link, 2016).

Link (2016) vertritt die Meinung, dass wir die Teilhabe behinderter Menschen verbessern könnten, wenn Behinderung als gesellschaftliche Aufgabe gesehen wird und nicht als individuelles Defizit. Dafür werden rechtliche Rahmenbedingungen benötigt und ein Teilhabegesetz, das behinderten Menschen ermöglicht, außerhalb von Heimen selbstbestimmt im eigenen Wohnraum zu leben, unabhängig vom Einkommen. 
„Wenn gesellschaftliche Teilhabe verwirklicht werden soll, muss öfter danach gefragt werden, wie Barrierefreiheit ermöglicht wird, anstatt den Zugang zu verweigern, denn behindert ist man nicht, sondern behindert wird man“(Link, 2016, S.1).

Bereits 50 Jahre vor Links Vortrag gab es von der britischen Union of the Physically Impaired Against Segregation (UPIAS) radikale Zweifel am medizinischen Modell von Behinderung und der rein auf individuelle Defizite gelenkte Blickwinkel auf Behinderung wurde in Frage gestellt. So wurde Behinderung neu als soziale Unterdrückung beschrieben, wie sie auch andere Gruppen, wie Frauen, oder Minderheiten, wie Homosexuelle, erfuhren und noch immer erfahren (Barnes, 2011). Das soziale Modell wird von Oliver, einem Unionsmitglied der UPIAS, folgendermaßen definiert:

„The social model involves nothing more or less fundamental than a switch away from focusing on the physical limitations of particular individuals to the way the physical and social environment impose limitations upon categories of people." (Oliver, 1981, S. 28 zit. n. Barnes, 2011, S. 62).

Das soziale Modell umfasst mehrere Prinzipien. Es werden die Wichtigkeit und Notwendigkeit von passenden individuellen Interventionen nicht abgesprochen. Auch beinhaltet es das Bestreben, die Aufmerksamkeit von den funktionellen Einschränkungen, die von der Umgebung, den Barrieren und Kulturen verursacht werden, wegzulenken auf eine Gestaltung der Umgebung, die für die größtmögliche Anzahl von Menschen passend gestaltet werden soll. Designer und Architekten richten ihre Aufmerksamkeit häufig nicht auf die Anforderungen aller Menschen. Die Lösung dieses Problems bietet das Universelle Design (Barnes, 2011).

Universelles Design bietet einen Ansatz, der nicht auf Defizite konzentriert ist und im Kapitel Barrierefreiheit und Universelles Design noch genauer beschrieben wird. Das Universelle Design bietet für Tourismusbetriebe eine Möglichkeit, ihre Zielgruppe zu erweitern, da alle Menschen davon einen Vorteil haben. Auch öffentliche Verkehrsanbieter und NutzerInnen können vom universellen Design profitieren.

Christiane Link hat daher den Verein „Transport for All“ gegründet, dessen Aufgabe es ist, den öffentlichen Verkehr für alle Menschen besser nutzbar zu machen (Link, 2016). 
„Menschen mit einer motorischen Behinderung können sich auch als nichtbehindert erleben und handeln. Eine Behinderung tritt erst im sozialen Bezug in Erscheinung. Behinderung ist daher nicht so sehr ein individuelles Problem, sondern ein weitreichend sozialund gesellschaftlich bestimmtes Phänomen“"(Leyendecker, 2005, S.103).

Dieses Zitat verdeutlich den hohen Stellenwert der sozialen Komponente von Behinderung und zeigt wie sehr Behinderungen durch die Gesellschaft bedingt sind.

Körperliche Behinderungen können die soziale Teilhabe, beispielsweise bei Reisen, einschränken (Leyendecker, 2005). Menschen mit motorischen Behinderungen bilden die Zielgruppe in der Befragung des empirischen Teils dieser Arbeit. Die Zielgruppe wird im Kapitel 7.2 beschrieben.

\section{Barrierefreiheit und Universelles Design}

Wenn Menschen mit körperlicher Behinderung verreisen, benötigen sie eine Umgebung, die das zulässt und barrierefrei gestaltet ist. Das kann beispielsweise durch universelles Design ermöglicht werden.

Das Wort Barriere leitet sich aus dem galloromanischen Wort „barra“ ab, das mit Querbalken übersetzt werden kann. Der Begriff der Barriere steht in Verbindung mit dem sozialen Modell von Behinderung. Im Kontext der Selbstbestimmungsbewegung von Menschen mit Behinderung wurde die Vorstellung entwickelt, dass Behinderung ein Ergebnis gesellschaftlicher Ausgrenzung ist und nicht primär mit dem Körper in Verbindung steht. Der Slogan der Selbstbestimmt-Leben-Bewegung war: People are disabled by society, not by their bodies! In diesem Bezug wird Behinderung als durch physische, soziale und ökonomische Barrieren gesellschaftlich bedingtes Behindertwerden gesehen. Der Begriff Barriere ist sozialrechtlich und sozialpolitisch von Bedeutung. In Gesetzestexten wird nur der positiv konnotierte Gegenbegriff der Barrierefreiheit angeführt (Kastl, 2017). 
Auch Heck beschreibt Barrierefreiheit nicht als Begriff für ein Fehlen von rein architektonische Zugangsbarrieren. Sie zielt auf den Abbau von benachteiligenden Behinderungen in einem umfassenden Sinn. Das beinhaltet auch physische, psychische, rechtliche, soziale, ökonomische, gesellschaftliche, bildungspolitische, kulturelle, informationelle, kommunikative und einstellungsbezogene Barrieren (Heck, 2012, zit. n. Wilken, 2015, S. 468).

Der Artikel 9 der UN-Behindertenrechtskonvention beinhaltet die Verwirklichung von Barrierefreiheit:

„Um Menschen mit Behinderungen ein selbstbestimmtes Leben und die volle Teilhabe in allen Lebensbereichen zu ermöglichen, treffen die Vertragsstaaten geeignete Maßnahmen mit dem Ziel, für Menschen mit Behinderungen gleichberechtigt mit anderen Zugang zur physischen Umwelt, zu Transportmitteln, Information und Kommunikation, einschließlich Informations- und Koтmunikationstechnologien und Systemen, sowie zu anderen Einrichtungen und Diensten, die der Öffentlichkeit in städtischen und ländlichen Gebieten offenstehen oder für sie bereitgestellt werden, zu gewährleisten. " (Sozialministerium, 2016).

Es wird die Ermöglichung von Zugängen in allen Bereichen beschrieben. Der Zugang zu öffentlichen Transportmitteln, Gebäuden und Einrichtungen soll durch Assistenzleistungen erleichtert werden, damit Barrierefreiheit gewährleistet werden kann. Es wird beschrieben, dass Assistenz bei Bedarf zur Verfügung gestellt wird (Sozialministerium, 2016). Die genaue Verwirklichung von persönlicher Assistenz wird im nächsten Unterkapitel ausgeführt, da für Menschen mit Behinderung diese notwendig sein kann, um eine Reise zu verwirklichen.

Die Vorstellung einer vollständigen barrierefreien Umwelt, wie vom Sozialministerium hier beschrieben, wird allerdings heute auch von ehemaligen Verfechtern des sozialen Modells in Frage gestellt, denn was für einen Menschen mit einer Gehbehinderung eine Barriere ist, kann für einen blinden Menschen zur Orientierung dienen. Barrierefreiheit ist immer das Ergebnis komplexer politischer Kompromisse und auch durch gesellschaftliche Möglichkeiten und Ressourcen begrenzt (Kastl, 2017). 
Kastl (2017) beschreibt die Unmöglichkeit einer universellen Barrierefreiheit. Das wirft die Frage auf, wie Barrierefreiheit nun für alle umgesetzt werden kann.

Es werden in den nächsten Kapiteln Lösungen präsentiert, die diese verschiedenen Anforderungen an Barrierefreiheit erfüllen können. Eine Möglichkeit bietet das Assistenzsystem und eine weitere wären zielgenaue Angaben, die im Best-Practice-Beispiel im Kapitel 4.2.1 ausgeführt werden. Um Barrierefreiheit auf Reisen zu gewährleisten ist es für Personen mit einer körperlichen Behinderung oft notwendig, persönliche Assistenz zu beanspruchen. Die Verwirklichung einer persönlichen Assistenz wird im folgenden Kapitel ausgeführt.

\subsection{Persönliche Assistenz}

Seit dem zweiten Weltkrieg gab es viele gesellschaftliche Veränderungsprozesse für Menschen mit Behinderung, die von völliger Marginalisierung und Euthanasie bis hin zu Empowerment mittels persönlicher Assistenz führten. Der Wandel, der sich von Fremdbestimmung bis hin zum selbstbestimmten Assistenzmodell vollzogen hat, wird in den nächsten Punkten kurz dargestellt.

- Zeit des Nationalsozialismus: Menschen mit Behinderung wurden getötet

- 1946 bis 1960: Verwahrung: Separation/Hospitalisierung/Leben in Anstalten: medizinisches Modell

- 1960-1990: Förderung: Sondereinrichtungen entstehen und die Enthospitalisierung beginnt

- Normalisierung: Menschen mit Behinderung sollen ein möglichst normales Leben führen können, beispielsweise einer Arbeit nachgehen.

- Entwicklung vom medizinischen Modell zum sozialen Modell (Weber, 2016). 
- Selbstbestimmt Leben und Empowerment:

Diese beiden Begriffe sind für eine barrierefreie Reisegestaltung essenziell. Hier geht es darum ein selbstbestimmtes Leben zu führen und Entscheidungen des eigenen Lebens so autonom wie möglich treffen zu können. Menschen mit Behinderung können selbst ihre Freizeit und Reiseaktivitäten planen. Folgende Fragen können selbst entschieden werden:

Wann und wo mache ich Urlaub?

Wen nehme ich mit? Welche Aktivitäten interessieren mich? Wer pflegt mich?

Dies kann durch persönliche Assistenz verwirklicht werden (Weber, 2016).

Das Assistenzmodell stellt das Gegenmodell zu Fürsorge und entmündigendem Versorgungsdenken dar. Unterstützungsleitungen für Menschen mit Behinderung waren im 20.Jh stets im Wandel und das ist auch nun wieder der Fall. Empowerment und Selbstbestimmung sind zentrale Begriffe, wenn es um Assistenzleistung geht. Durch diese Theorien wurde es möglich ein Assistenzmodell für Menschen mit Behinderung einzuführen (Weber, 2016).

Das Empowerment-Konzept wurde in den USA entwickelt. Die Bekanntheit von Empowerment stieg in der US-amerikanischen Bürgerrechtsbewegung. Die Bewegung war mit kollektiven Aktionen gegen Diskriminierung, gesellschaftliche Benachteiligung und Segregation von der Zivilgesellschaft selbstorganisiert. Empowerment kann als Selbstermächtigung oder Selbstbefähigung übersetzt werden. Jedoch beschreibt Theunissen (2016), dass diese Übersetzung Empowerment nicht ausreichend erfasst. Er verbindet diesen Begriff mit Behindertenpädagogik und sozialer Arbeit, die er mit vier Zugängen beschreibt:

1. Individuumsbezogener Zugang

Es wird von der eigenen Lebenskraft und Stärke einer Person und ihre individuellen Ressourcen ausgegangen. 


\section{Kollektiver Zugang}

Dieser bezieht sich auf einen Zusammenschluss von Menschen in schwierigen Lebenslagen, die ihre Angelegenheiten selbst in die Hand nehmen und sich dabei ihrer Macht und Potentiale bewusst sind und sich in Eigeneregie Wissen und Fähigkeiten aneignen.

3. Politischer Zugang

Hierbei handelt es sich um die Thematisierung sozialer Benachteiligung, Diskriminierung und ungleicher Machtverhältnisse, die durch gemeinsame Widerstandskräfte und politische Aktionen überwunden werden können.

\section{Unterstützender Zugang}

Der vierte Punkt beschreibt die professionelle Aufgabe, Menschen in einer marginalisierten Position in ihrer Lebenskraft zu stärken und zu einer Selbstvertretung zu befähigen. Die unterstützende Person soll in schwierigen Lebenslagen assistierend beiseite stehen (Theunissen, 2016).

Selbstbestimmung bzw. Autonomie gelten bereits seit den 1990er Jahren als Leitprinzipien in der Arbeit mit Menschen mit Behinderung und hat das Prinzip der Fürsorge abgelöst. Menschen mit Behinderung haben nach dem Vorbild anderer benachteiligter Gruppen, wie beispielsweise Arbeiter, Frauen oder ethnische Minderheiten, begonnen, elementare Bürgerrechte zu fordern, gegen die Exklusion zu kämpfen und sich für ein selbstbestimmtes Leben einzusetzen (Dederich, 2016).

Ein Grund für die Umsetzung der Empowerment- und Selbstbestimmungsidee ist der Auftrag der UN-Behindertenrechtskonvention. Hier werden Staaten zum Aufbau von Unterstützungssystemen nach dem Assistenzmodell verpflichtet. Die Vertragsstaaten haben dafür zu sorgen, dass Menschen mit Behinderung Zugang zu einer Reihe von Unterstützungsdiensten haben. Diese sollten sich in der Nähe der Gemeinde befinden und persönliche Assistenz beinhalten (Sozialministerium, 2016). 
Persönliche Assistenz wird als Hilfestellung zur Bewältigung des täglichen Lebens verstanden. Im Fokus steht hier, ein Leben nach eigenen Vorstellungen mit möglichst wenig Fremdbestimmung zu gestalten. AssistenznehmerInnen entscheiden eigenverantwortlich, wann und wie sie ihre Unterstützungsleistungen nützen. Die Art, der Umfang und der Inhalt der persönlichen Assistenz richtet sich immer nach der jeweiligen Lebenssituation der AssistenznehmerInnen. Persönliche AssistentInnen ersetzen die Motorik eines Menschen mit Behinderung. Der Lebensalltag wird von der Person selbst gestaltet. HilfeempfängerInnen sind gleichzeitig der Arbeitgeber oder die Arbeitgeberin (Franz, 2002).

Zur Verwirklichung dieses Modells ist es notwendig, dass AssistenznehmerInnen über vier Kompetenzen verfügen:

1. Personalkompetenz

2. Anleitungskompetenz

3. Organisationskompetenz

4. Finanzkompetenz

Menschen mit Behinderung entscheiden, wer ihnen assistiert und organisieren Zeitpunkt, Ort und die Finanzierung (Rock, 2001).

Es besteht auch die Möglichkeit, sich Assistenz über einen Verein organisieren zu lassen (Organisation, Stundenpläne, Bezahlung usw.) (Franz, 2002).

In Graz gibt es beispielsweise den Verein „Wegweiser“, der Menschen mit Behinderung dabei unterstützt, persönliche Assistenz in Anspruch zu nehmen.

Assistenzleistungen auf Reisen sind Teil der für diese Arbeit durchgeführten Studie. Dies dient zur Beantwortung der Frage: Welche Möglichkeiten zur Realisierung einer Reise werden von Menschen mit Behinderung genutzt?

Im nächsten Kapitel wird eine Möglichkeit beschrieben, persönliche Assistenz zu beziehen. 


\subsubsection{Persönliches Budget}

ReiseassistentInnen können über persönliches Budget finanziert werden, damit zu den Reisekosten nicht zusätzlich noch Lohn für AssistentInnen aus der privaten Kasse anfällt.

Es handelt sich beim persönlichen Budget um eine Geldleistung, die direkt an die Person mit einer körperlichen Behinderung bezahlt wird, damit diese persönliche Assistenz beziehen kann. Dadurch soll ein maximales Ausmaß an Selbstbestimmung und individueller Lebensgestaltung ermöglicht werden (Land Steiermark, 2018).

Die Internetseite des Landes Steiermark (2018), beschreibt welche Voraussetzungen für Persönliches Budget notwendig sind. Wenn ein Mensch mit Behinderung folgende Kompetenzen erfüllt, liegt gleichzeitig ein Rechtsanspruch dafür vor (hier am Beispiel Steiermark):

- geschäftsfähige Person

- Person mit Sinnesbeeinträchtigungen und/oder erheblichen Bewegungsbehinderungen

- vollendetes 18. Lebensjahr

- österreichische Staatsbürgerschaft oder EWR-Staatsbürgerschaft bzw. Aufenthaltserlaubnis bzw. Niederlassungsbewilligung nach dem Niederlassungs- und Aufenthaltsgesetz

- Hauptwohnsitz in der Steiermark (Land Steiermark, 2018).

Die gleichzeitige Inanspruchnahme von „Persönlichem Budget“ mit mobilen Diensten der Behindertenhilfe und stationären Wohneinrichtungen ist nicht möglich. Das persönliche Budget kann für jede Form der persönlichen Hilfen in den folgenden Bereichen eingesetzt werden:

- Haushalt,

- Körperpflege und Grundbedürfnisse,

- Erhaltung der Gesundheit, Mobilität,

- Kommunikation und

- Freizeit (Land Steiermark, 2018). 
Der Bereich Freizeit zeigt sich für eine barrierefreie Reise essenziell, da das persönliche Budget unter anderen dafür da ist, sich eine Assistenzleistung für eine Reise zu organisieren. Jemand mit persönlichem Budget erfüllt daher die rechtlichen Rahmenbedingungen, um sich Assistenz für eine Reise zu organisieren und es werden die Gelder für die Reiseassistenz zur Verfügung gestellt. Die betroffenen Menschen entscheiden innerhalb des vorgegebenen Rahmens selbst über den Leistungszukauf, das heißt, wen sie zur persönlichen Assistenz heranziehen und wie hoch die Vergütung dafür erfolgt. Auf Reisen kann ein Stundenlohn oder eine Pauschale vor Reiseantritt ausgehandelt werden. Unterhaltspflichtige Angehörige und andere Angehörige, die mit dem Menschen mit Behinderung in einem gemeinsamen Haushalt leben, können nicht als AssistentInnen herangezogen werden. Die Jahresstunden werden mit einem Stundensatz in der Höhe von 24,20 Euro multipliziert. Das persönliche Budget wird nach Leistungszuerkennung vierteljährlich im Vorhinein an die Menschen mit Behinderung ausgezahlt. Sie haben dafür ein ausschließlich für das persönliche Budget vorgesehenes Konto einzurichten (Land Steiermark, 2018).

Das persönliche Budget bietet vielen Menschen in Österreich eine Hilfeleistung selbstbestimmt barrierefreie Reisen zu organisieren. Das nächste Kapitel behandelt konkret wie Barrierefreiheit in Österreich normiert wird.

\subsection{Barrierefreiheit in Österreich}

Barrierefreiheit in Österreich ist trotz des hohen wirtschaftlichen Potentials erst wenig quantitativ erforscht. Eine Ausnahme bildet die Arbeit von Rannegger (2006), mit dem Ergebnis, dass knapp ein Drittel (31,2\%) der Menschen mit körperlicher Behinderung aus Österreichs innerhalb des eigenen Landes reist. Es ist mit Abstand das beliebteste Reiseziel. An zweiter Stelle liegt Kroatien mit 13, 7\%. Dies zeigt die Bedeutsamkeit touristischer Betriebe in Österreich für Menschen mit Behinderung auf. Barrierefreiheit in Österreich ist messbar durch die Ö-Norm, welche in diesem Kapitel beschrieben und mit einem praktischen Beispiel veranschaulicht wird. 
Wenn in Österreich direkt gereist wird, gibt es bestimmte Normen, die Ö-Normen, welche von barrierefreien touristischen Betrieben eingehalten werden müssen. Barrierefreiheit ist für alle Menschen mit Behinderung individuell definiert, da die Anforderungen an eine barrierefreie Umgebung von Person zu Person variieren (Kastl, 2017). Es muss jedoch allgemeine Regelungen geben, damit barrierefreies Bauen ermöglicht wird. Diese unterscheiden sich nun wieder zwischen verschiedenen Staaten. In Österreich gibt es hierfür die Ö-Norm, in Deutschland beispielsweise die DIN-Norm, was bedeutet, dass in Österreich andere Maßstäbe gelten, als in Deutschland und die Werte dadurch variieren. Für Reisende mit Behinderung bedeutet dies, dass die normierte Definition von Barrierefreiheit in Österreich für ihre Bedürfnisse passen kann, in einem anderen Land wie Deutschland allerdings nicht. Barrierefreie Sanitärräume sind für viele Teilbereiche des barrierefreien Tourismus, wie beispielsweise Unterkünfte, Freizeitaktivitäten und An- und Abfahrt notwendig. Aus diesem Grund wird zur Veranschaulichung der Ö-Norm, die vorgegebenen Maßstäbe für die Einrichtung eines WC-Sitzes beschrieben. Das Beispiel wurde gewählt, da in der Studie von Flucher, Parigger \& Zirngast (2013) die häufigsten Probleme auf Reisen von Menschen mit Behinderung im Zusammenhang mit sanitären Anlagen auftreten. Auch Rannegger (2006) beschreibt, dass die Sanitärmöblierung als größte Schwierigkeit in den meisten Kettengliedern der touristischen Servicekette genannt wird, etwa in der Unterkunft.

„Die Vorderkante des WC-Sitzes muss von der anzufahrenden Rückwand einen Abstand von mindestens $65 \mathrm{~cm}$ haben. Für das seitliche und rechtwinkelige Anfahren (ein-oder Beidseitig) ist eine Mindestfläche mit einem Abstand von $90 \mathrm{~cm}$ zwischen seitlicher Kante der Toilette und Wand und $120 \mathrm{~cm}$ vor der WC-Schale einzuhalten. Generell sollte die Sitzhöhe des WC-Sitzes $46 \mathrm{~cm}$ betragen, darf jedoch auf keinen Fall $48 \mathrm{~cm}$ überschreiten. Ab einer Sitztiefe von $55 \mathrm{~cm}$ muss eine möglichst wärmegedämmte Anlehnfläche in der Breite des WC-Sitzes in einer Höhe von $60 \mathrm{~cm}$ vorgesehen werden. Die WC-Sitzbefestigung muss so stabil sein, dass mit einer an der Vorderkante der Sitzbrille seitlich angreifenden Kraft von 750 N, die Sitzbrille um nicht mehr als $1 \mathrm{~cm}$ seitlich verschoben werden kann" (Österreichisches Normungsinstitut, 2005, S. 24). 


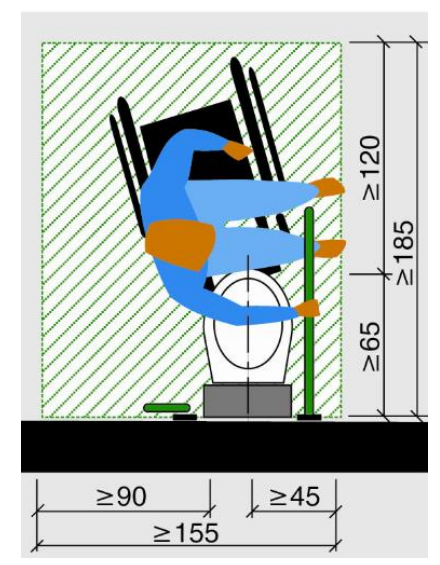

Abbildung 2: Österreichisches Normungsinstitut (2005).

\subsection{Universelles Design}

Einen Lösungsansatz für die Verwirklichung von Barrierefreiheit und damit eine nötige Voraussetzung für das Verreisen von Menschen mit Behinderung bietet das Konzept des universellen Designs.

Universelles Design führt zu Barrierefreiheit, ohne auf spezielle Defizite der KundInnen einzugehen. Der Ansatz des Universellen Designs ist positiv formuliert. Das Design des Produkts, der Infrastruktur, der Umgebung oder des Kommunikationssystems soll für alle Menschen nutzbar sein, mit größtmöglicher Ausbreitung und ohne dafür eine Adaption speziell für gewisse Behinderungen zu benötigen (Barnes, 2011).

Die Entwicklung des universellen Designs steht direkt in Verbindung mit dem sozialen Modell, das im Kapitel 2.2 definiert wurde.

Universelles Design bietet einen Ansatz, der nicht auf Defizite konzentriert ist, wie es beim medizinischen Modell der Fall ist, sondern auf die Anforderungen der Nutzung durch alle Menschen fokussiert (Barnes, 2011).

Die hohe Relevanz wird von (Wylde, 1994 zit. n. Barnes, 2011, S. 69) aufgezeigt. Er beschreibt, dass neun von zehn Personen in einer Station des Lebens Diskriminierung erfahren, die von der architektonischen Umgebung ausgelöst wird. 
Universelles Design basiert auf den folgenden Prinzipien:

- Breite Nutzbarkeit: Das Design ist für Menschen mit unterschiedlichen Fähigkeiten nutzbar und marktfähig. Beispiele hierfür sind automatische Eingangstüren mit Sensoren, die für alle Menschen gut erreichbar und bedienbar sind oder anpassbare Sitzgelegenheiten.

- Flexible Nutzung: Das Design bietet eine große Bandbreite für individuelle Vorzüge und Fähigkeiten. Ein Beispiel ist der Museumsbesuch mit der Möglichkeit eines Audioguides.

- Einfachheit und intuitive Nutzbarkeit: Die Benutzung des Designs soll leicht verständlich und unabhängig von der Erfahrung der NutzerInnen sein. Ein Beispiel hierfür wäre eine rein grafische Darstellung ohne Text bei einer Bedienungsanleitung.

- Leicht verständliche Gebrauchsinformation. Die notwendige Information wird den Nutzern effektiv kommuniziert, ohne speziell Bezug auf sensorische Fähigkeiten zu nehmen. Taktile, auditive und visuelle Rasterungen eines Gebrauchsgegenstandes führen zu einer Erleichterung der Bedienung. Ein weiteres einfaches Beispiel dafür bieten Videos mit Untertiteln.

- Fehlertoleranz: Das Design reduziert Gefahren bei der Bedienung und reduziert dabei auch negative Konsequenzen, die bei Unfällen entstehen können.

- Wenig physischer Aufwand: Beispielsweise können hier Türen mit elektronischen Türöffnern genannt werden.

- Größe und Platz für Zugang und Benutzung: Zum Beispiel eine Benutzung unabhängig von der Größe der BenutzerInnen bei Zugangskontrollen von Bahnsteigen und verstellbare Arbeitstische in Büros (Fissler, 2015).

Universelles Design soll Barrierefreiheit und Zugang für Menschen bieten, ohne dass Alter, Behinderung, Geschlecht, Ethnizität von Bedeutung sind. Es soll möglichst vielen Menschen ermöglicht werden, ihre täglichen Aktivitäten komfortabel und sicher zu bewältigen. Gebäude mit Rampen und Aufzügen sind nicht nur von Vorteil für Menschen mit Behinderung, sondern beispielsweise auch für Eltern mit Kinderwagen (Barnes, 2011). 
Die Idee des Universellen Designs wird im barrierefreien Tourismus übernommen. Der Verein „atempo“ aus Graz evaluiert touristische Betriebe auf ihre Barrierefreiheit. Dabei wird nicht nur die Tauglichkeit für RollstuhlfahrerInnen, sondern auch für Eltern mit Kinderwagen, für AllergikerInnen u. a. überprüft bzw. berücksichtigt. Die genauere Beschreibung folgt in Kapitel 4.2.2.

Auf der Touristischen Servicekette gibt es viele Beispiele, an denen Universelles Design von Vorteil ist. Im nächsten Kapitel soll die touristische Servicekette beschrieben und mit praktischen Realisierungsmöglichkeiten des Universellen Designs ergänzt werden.

\section{Barrierefreier Tourismus}

Dieses Kapitel beinhaltet, am Beispiel der touristischen Servicekette, in welche Problembereiche der barrierefreie Tourismus aufgeteilt werden kann. Best Practice Beispiele sollen einen Einblick geben, wie barrierefreier Tourismus funktionieren kann.

Die meisten Menschen mit Behinderung in Österreich bleiben beim Reisen innerhalb des eigenen Staates. Auf Platz zwei liegt Kroatien und auf Platz drei Italien. Die durchschnittliche Reisedauer beträgt 10 Nächtigungen und 95 Prozent Verreisen mit Begleitung. Über die Hälfte machen den Urlaub zu zweit oder zu dritt.

Wilken (2016) beschreibt Barrierefreiheit als etwas, das für 10\% der Menschen dringend erforderlich, für 30\% hilfreich und für 100\% komfortabel ist. Eltern mit Kinderwagen, Menschen im Rollstuhl und allen Menschen bietet barrierefreier Tourismus Vorteile. Wilken beschreibt eine Steigerungsfähigkeit des Umsatzpotentials des barrierefreien Tourismus in Europa von ca. 16 Milliarden Euro. Trotz dieser Entwicklungsprognose meiden nicht wenige touristische Betriebe ein offensichtliches Engagement für den barrierefreien Tourismus. Es wird hier oft genannt, dass die absoluten Ansprüche und die Vielseitigkeit von Behinderung verunsichern und demotivieren. Wilkens Forschung zeigt jedoch das hohe Potential, das im barrierefreien Tourismus noch ausgeschöpft werden kann. 
Der Idee des barrierefreien Tourismus für alle liegt das Konzept des universellen Designs zugrunde, das auf gesamteuropäischer Ebene erarbeitet wurde. Universelles Design versteht sich als ein Prinzip zur (Um-) Gestaltung von Umwelt, Produkten und Dienstleistungen, so dass alle Menschen in der Lage sind, gleichberechtigt an den gesellschaftlichen Aktivitäten teilzuhaben (EUCAN, 2003). Universelles Design wird im vorherigen Kapitel beschrieben.

Aus dieser Planungsphilosophie wurde die Definition des „Tourismus für Alle“ abgeleitet:

„Jede Person sollte unabhängig vom Alter oder einer Behinderung in der Lage sein zu reisen - in ein Land, innerhalb eines Landes oder zu der Destination, Sehenswürdigkeit oder Veranstaltung, die sie besuchen möchte“ (ENAT, 2007, S. 8).

\subsection{Die Touristische Servicekette}

Die touristische Servicekette ist ein Bestandteil österreichischer wissenschaftlicher Arbeit zum Thema barrierefreien Reisen. Sie wurde als Reiseaufteilung von Rannegger (2006), Höglinger (2010) und Flucher, Parigger \& Zirngast (2013) verwendet. Es wird jeweils als Quelle der ADAC angegeben, die auch für diese Arbeit herangezogen wird. In den Recherchen dieser Arbeit konnte die Herkunft des Begriffs nicht genauer nachvollzogen werden, da in österreichischen und deutschen Quellen der ADAC zitiert wird, dieser jedoch keine Auskunft zu etwaigen anderen Quellen liefert. Es ist daher unklar, ob der ADAC selbst Urheber des Begriffs ist oder ihn von anderer Stelle übernommen hat.

Es ist notwendig die Schritte einer Reise aufzuteilen, da in jeder Station andere Bereiche essenziell sind. Die Teilbereiche der Servicekette greifen auch ineinander, wie an folgender Grafik ersichtlich ist: 


\section{Erwartungen}

\section{Betrachtung der gesamten Servicekette}

An- und Abreise

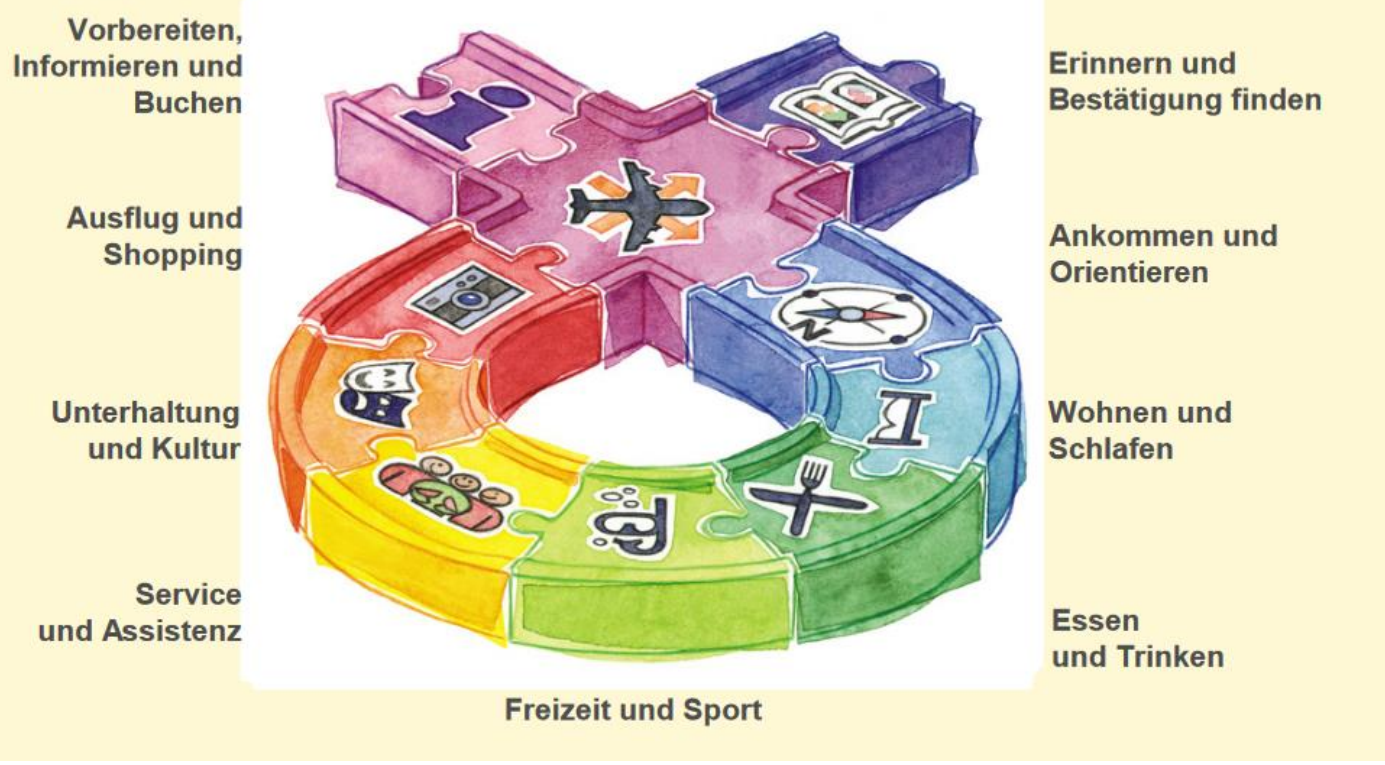

Abbildung 3: Die Touristische Servicekette (Pfeiffer, 2010).

Die Touristische Servicekette beginnt bei i, auf der Abbildung 3 links oben. Dieses i steht für Informieren, Vorbereiten und Buchen. Das nächste Element zeigt den Schritt An- und Abreise. Darauf folgt Ankommen und Orientieren, Wohnen und Schlafen, Essen und Trinken, Freizeit und Sport, Service und Assistenz, Unterhaltung und Kultur, Ausflug und Einkaufen und zuletzt Erinnern und Bestätigung finden. Diese Servicekette ist grundsätzlich für alle Menschen gleich. Der Unterschied besteht darin, dass UrlauberInnen mit Behinderung spezifischere Anforderungen an die Servicekette und deren Unterkategorien stellen. Bereits die Wahl eines Urlaubsortes kann Schwierigkeiten beinhalten. Viele MitarbeiterInnen von touristischen Informationsstellen und Reisebüros haben wenig Informationen über die Anforderungen einer barrierefreien Reise und welche Angebote in Frage kommen würden ADAC (2003).

Viele Broschüren und Internetseiten von Reiseanbietern gehen nur wenig oder gar nicht auf die Belange von KundInnen mit Behinderung ein. Die Urlaubsplanung für Menschen mit Behinderung benötigt daher einen höheren Zeitaufwand (ADAC, 2003). 
Die Anreise ist für Menschen mit einer körperlichen Behinderung mit einem höheren Aufkommen von Schwierigkeiten verbunden, wie beispielsweise Barrieren bei Verkehrsmitteln wie Bahn, Bus und Flugzeug. Die Anreise ist daher mit einem höheren Organisations- und Zeitaufwand verbunden (ADAC, 2003).

In dieser Arbeit werden die verschiedenen Kettenglieder zusammengefasst in die Bereiche Planung, An- und Abreise, Unterkunft und Freizeitangebot, das später auch in der Fragebogenbeschreibung ersichtlich wird.

\subsection{Praktische Realisierungsmöglichkeiten}

In diesem Kapitel werden praktische Umsetzungen beschrieben, die mit der touristischen Servicekette in Verbindung stehen. Zuerst werden Beispiele des universellen Designs genannt und es wird aufgezeigt, dass in allen Bereichen der touristischen Servicekette Umsetzungsmöglichkeiten bestehen. Die letzten beiden Unterkapitel behandeln Plattformen, die eine barrierefreie Reiseplanung ermöglichen.

\subsubsection{Universelles Design und die touristische Servicekette}

Kettenglied An- und Abreise

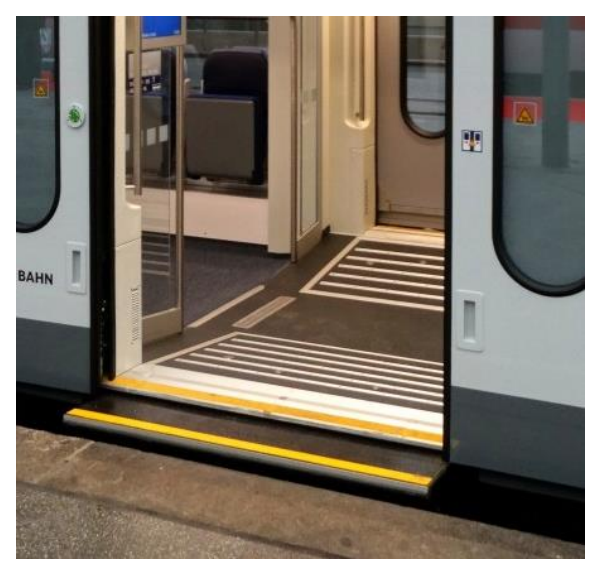

Abbildung 4: Deutsche Bahn. (2018).

Barrieren bei Transportmitteln können für Menschen mit körperlicher Behinderung schwer zu überwinden sein. Der hier gezeigte Zugeinstieg erfüllt alle Prinzipien des Universellen Designs und ermöglicht somit für viele Menschen einen einfachen Einstieg, wenn sie sich auf Reisen befinden. 


\section{Kettenglied Essen und Trinken}
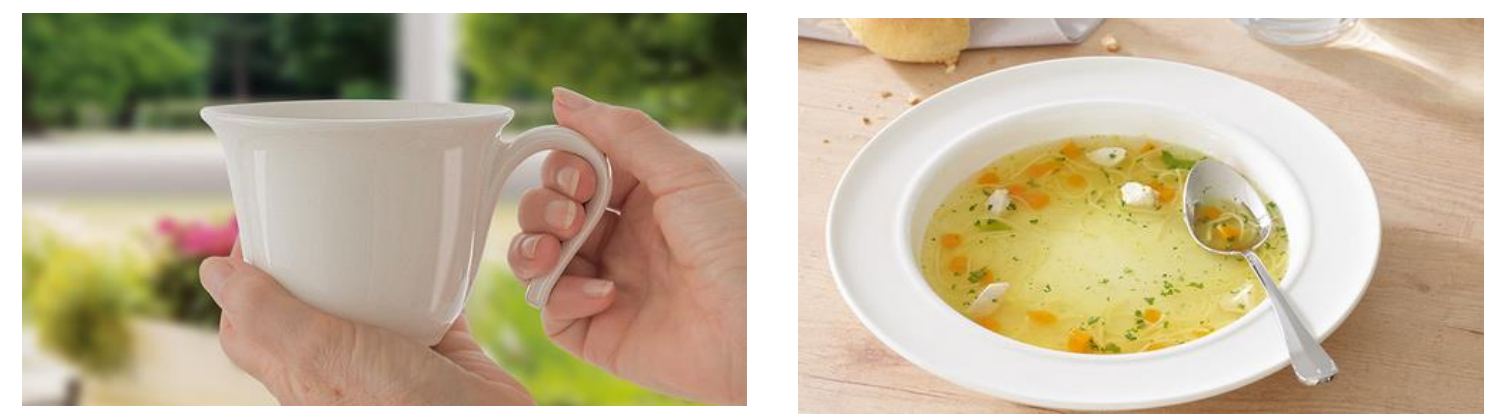

Abbildung 5: Bayrisches Staatsministerium für Familie, Arbeit und Soziales (2017).

Der Henkel der Tasse ist unten offen und damit leicht $\mathrm{zu}$ greifen und sie besitzt eine Wärmedämmung, damit Verbrennungen vermieden werden. Das Material ist belastbar. Der ausgestellte Becherrand erleichtert das Trinken. Der Teller hat steile Seitenwände und eine Abstreifkante, um den Löffel leichter führen zu können.

In der Mitte befindet sich eine Wölbung, damit die Suppe bis zum letzten Löffel ohne Probleme aufgenommen werden kann.

\section{Kettenglied Wohnen und Schlafen}

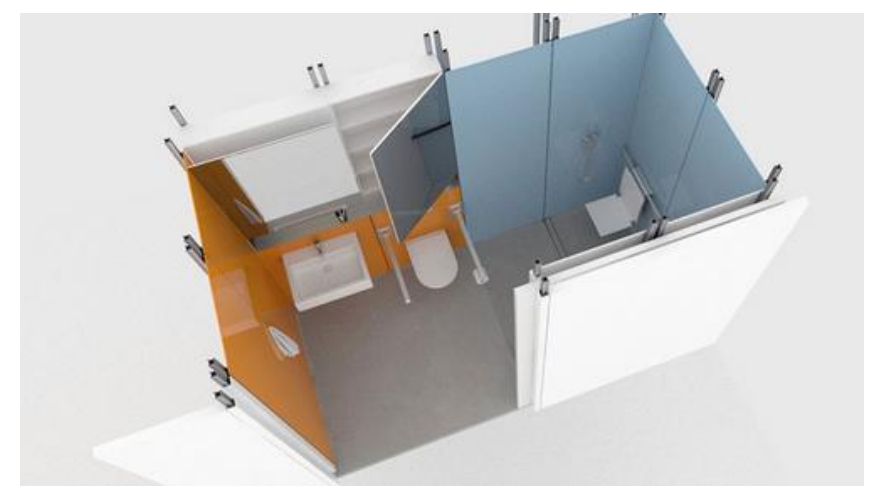

Abbildung 6: Bayrisches Staatsministerium für Familie, Arbeit und Soziales (2017).

Das Systembad bietet den nötigen Platz für einen Rollstuhl im Duschbereich. Eine große Schiebetür schafft die nötige Bewegungsfläche für einen Rollstuhl. Das Waschbecken ist unterfahrbar und Haltegriffe sind beidseitig bei der Toilette vorhanden. Es werden hier wieder alle sieben Prinzipien des Universellen Designs erfüllt. Das Bad kann sehr einfach mit anderen Systemen umgebaut werden, je nachdem, ob ein Rollstuhl verwendet wird oder nicht. 


\section{Kettenglied Freizeit und Sport}

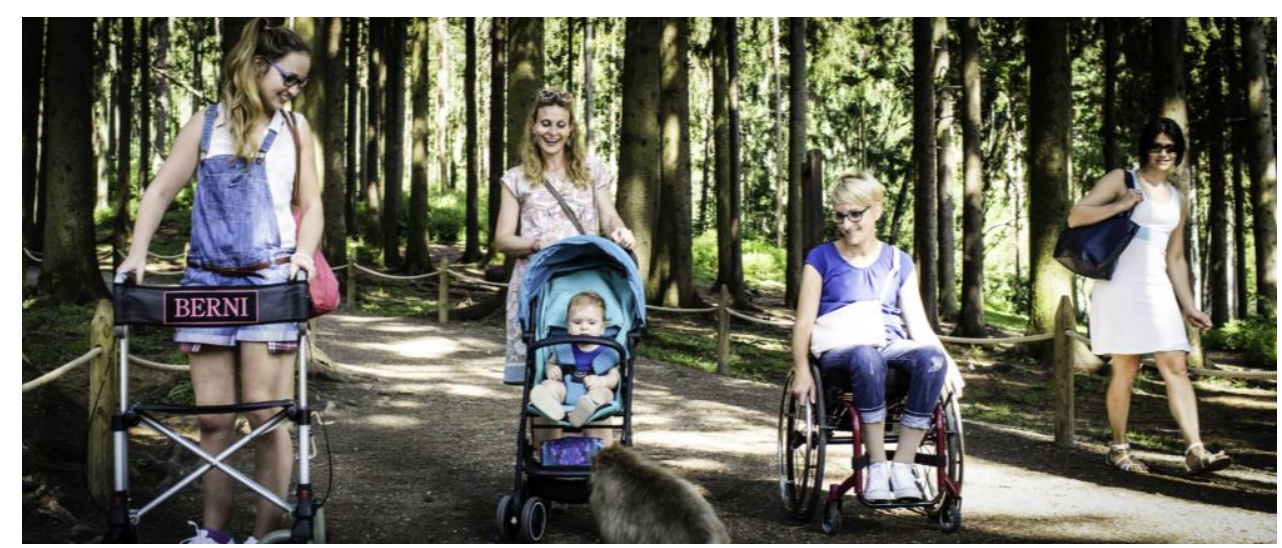

Abbildung 7: Fojan (2018).

Hier wird ein angelegter Wanderweg abgebildet, der für alle Menschen gut nutzbar ist. Es können alle Personen gemeinsam an der Freizeitaktivität teilnehmen, egal ob sie auf ein Unterstützungsmittel angewiesen sind oder nicht.

\section{Kettenglied Unterhaltung und Kultur}

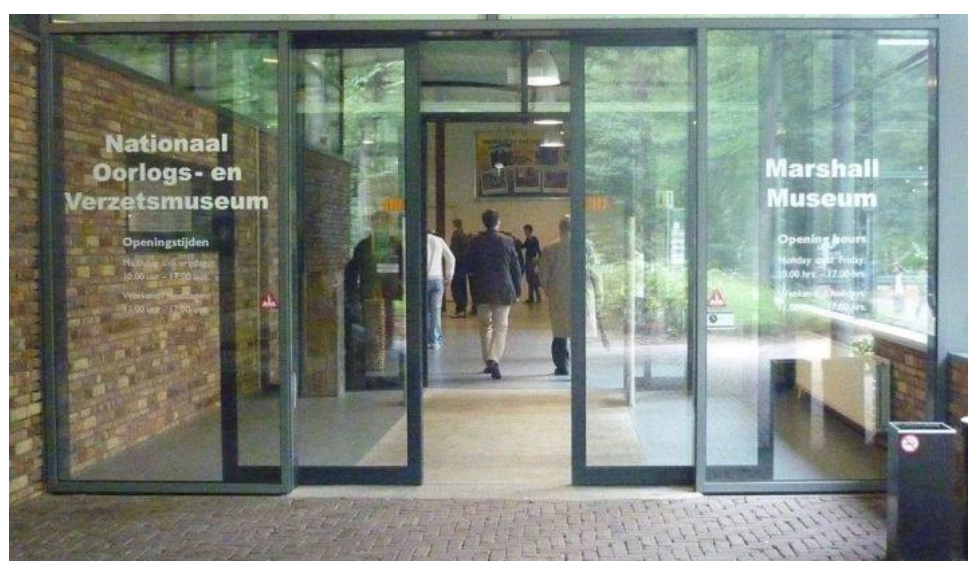

Abbildung 8: Oorlogsmuseum (2017).

Automatische Eingangstüren mit Sensoren sind für Menschen mit unterschiedlichen Fähigkeiten nutzbar. In öffentlichen Gebäuden, Museen oder anderen touristischen Gebäuden bieten sie eine gute und einfache Möglichkeit, einen barrierefreien Zugang zu schaffen und bieten damit wieder ein Design für alle.

Die Reiseplanung ist ein wichtiger Bestandteil barrierefreien Reisens, da im Vorfeld bereits etwaige Barrieren vermieden werden können. Das nächste Kapitel zeigt Online-Anwendungen, die bei der Reiseplanung hilfreich sein können. 


\subsubsection{Bereit für Barrierefreiheit}

Um eine barrierefreie Reise planen zu können, ist es notwendig, im Vorfeld zu klären, ob die Unterkunft barrierefrei ist und den individuellen Ansprüchen genügt. Der steirische Verein „atempo“ hat eine eigene Internetseite mit dem Namen „Bereit für Barrierefreiheit" erstellt. Es werden Experten und Expertinnen geschult, die vor Ort die jeweilige touristische Einrichtung vermessen. Es haben sich zahlreiche Gemeinden, Institutionen und touristische Einrichtungen mit dem Ziel zusammengeschlossen, größtmögliche Barrierefreiheit in ihren Organisationen, Betrieben, Einrichtungen und Räumen zu verwirklichen. Knotenpunkt dafür bietet das Social Franchise Unternehmen „capito“, das 2001 vom Grazer Verein „atempo“ gegründet wurde. Bei der Recherche für den nächsten Urlaub kann bei der Suche die jeweilige Zielgruppe im Vorfeld angegeben werden. Die Internetseite ist barrierefrei gestaltet, das bedeutet, dass Schriften und Kontraste einfach angepasst werden können (Bereit für Barrierefreiheit, 2017),

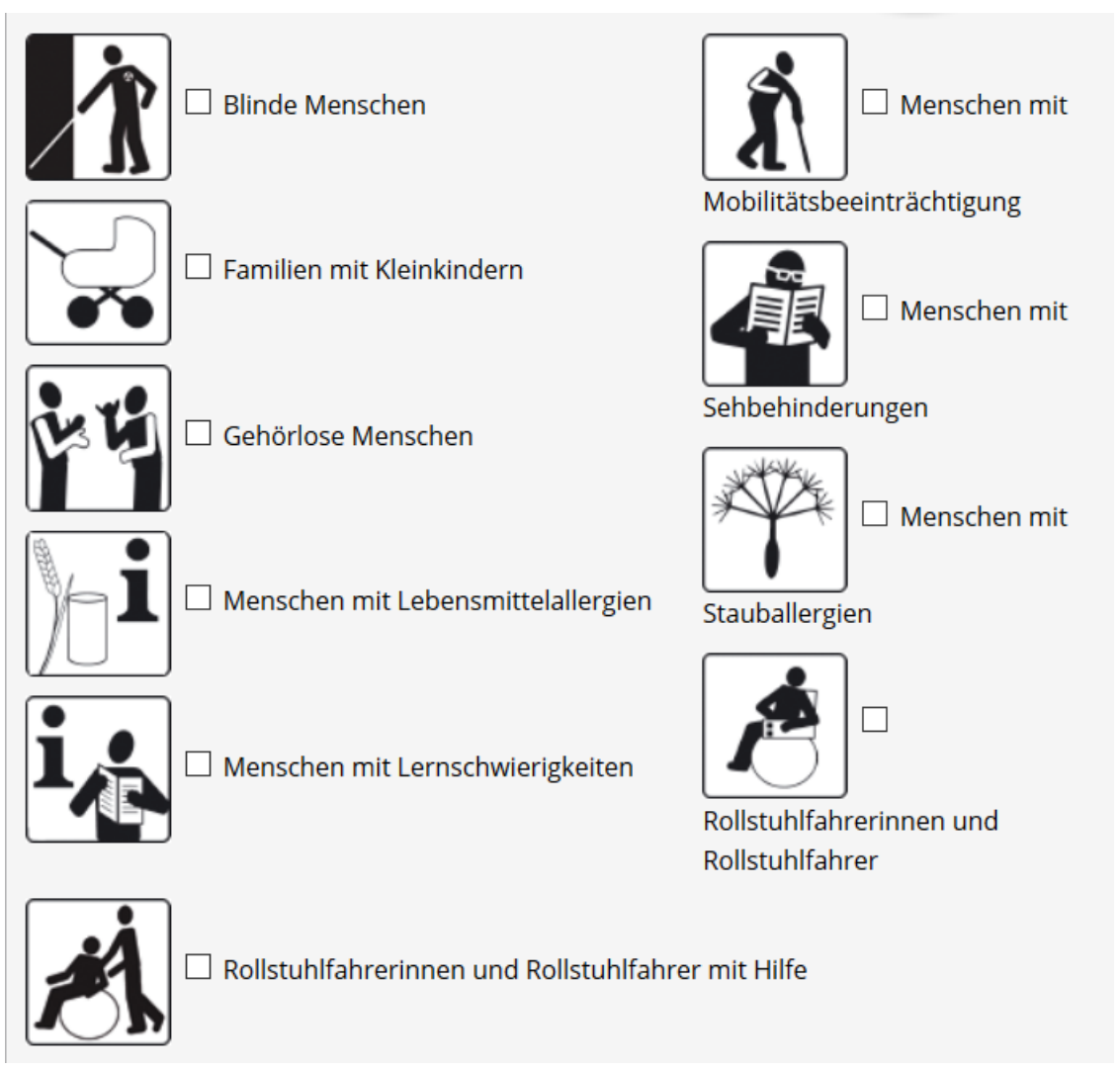

Abbildung 9: Bereit für Barrierefreiheit (2017). 
Bei der Abbildung wird gezeigt, für welche Zielgruppen die touristischen Angebote vermessen wurden. Die Unterkunft wird in Bezug auf ihre Barrierefreiheit und damit verbundene Informationen beschrieben. Zum Beispiel:

„Über eine Rampe kommt man vom Keller in den Lift und so erreicht man die Rezeption, das Restaurant sowie die barrierefreien Zimmer. Bei zwei Zimmern wurde besonders groBen Wert auf die Barrierefreiheit gelegt. Diese befinden sich im Stammhaus“ (Bereit für Barrierefreiheit, 2017).

Es besteht die Möglichkeit auf den gesamten Vermessungsbogen direkt auf der Webseite zuzugreifen. Hier werden die genauen Vermessungsangaben ersichtlich und es besteht die Möglichkeit ganz genau nachzusehen, ob die eigenen Anforderungen erfüllt werden können. Beispielsweise, ob das Waschbecken unterfahrbar ist oder ob Haltegriffe vorhanden und in welcher Höhe diese angebracht sind. Die folgende Grafik ist von „Bereit für Barrierefreiheit“" und beinhaltet einen Teil der Vermessungen eines Hotels (Bereit für Barrierefreiheit, 2017). 


\begin{tabular}{|c|c|}
\hline $\begin{array}{l}\text { Wenn es eine Notglocke gibt: ist diese vom WC aus sitzend (ca. in einer Höhe von } 70 \text { bis } 100 \mathrm{~cm} \text { ) } \\
\text { und vom Boden aus in einer Höhe von max. } 35 \mathrm{~cm} \text { über dem Boden erreichbar? [No] }\end{array}$ & JA \\
\hline $\begin{array}{l}\text { Gibt es im WC eine Notglocke? (Angaben zum Notruf: Wohin führt dieser? Wer kann Hilfestellung } \\
\text { leisten? Wie kann der Notruf quittiert werden?) [No] }\end{array}$ & JA \\
\hline $\begin{array}{l}\text { Befindet sich die Bedienebene von Ausstattungsgegenständen auf einer Höhe von } 80 \text { bis } 110 \mathrm{~cm} \text { ? } \\
\text { [No] }\end{array}$ & JA \\
\hline $\begin{array}{l}\text { Wenn es einen Spiegel gibt: ist dieser verstellbar oder reicht er bis zur Waschbeckenkante } \\
\text { (ÖNORM B 1600: Unterkante mind. } 95 \mathrm{~cm} \text { )? [Ex] } \\
\text { Anmerkung: verstellbar }\end{array}$ & JA \\
\hline $\begin{array}{l}\text { Ist der Siphon beim Waschbecken aus Kunststoff oder mit Porzellan geschützt? (ÖNORM B 1600: } \\
\text { Unterputz- oder Flachaufputz-Siphon) [Ex] } \\
\text { Anmerkung: Kunststoff }\end{array}$ & JA \\
\hline Beträgt die freie Höhe unter dem Waschbecken mind. $70 \mathrm{~cm}$ ? [No] & JA \\
\hline Beträgt die Mittelachse des Waschbeckens zur angrenzenden Wandfläche mind. $50 \mathrm{~cm}$ ? [No] & JA \\
\hline $\begin{array}{l}\text { Ist vor dem Waschbecken eine Bewegungsfläche von } 150 \mathrm{~cm} \times 150 \mathrm{~cm} \text { (Unterfahrbarkeit des } \\
\text { Waschbeckens bis max. } 20 \mathrm{~cm} \text { Tiefe kann miteinbezogen werden)? [No] }\end{array}$ & JA \\
\hline $\begin{array}{l}\text { Ist vor dem Waschbecken eine Bewegungsfläche von } 120 \mathrm{~cm} \times 120 \mathrm{~cm} \text { (Unterfahrbarkeit des } \\
\text { Waschbeckens bis max. } 20 \mathrm{~cm} \text { Tiefe kann miteinbezogen werden)? (ÖNorm B 1600: mind. } 150 \mathrm{x} \\
150 \mathrm{~cm} \text { ) [Ex] }\end{array}$ & JA \\
\hline $\begin{array}{l}\text { Ist das Waschbecken in einer Höhe von } 80 \text { bis } 85 \mathrm{~cm} \text { (Oberkante) montiert? [No] } \\
\text { Anmerkung: } 85 \mathrm{~cm}\end{array}$ & JA \\
\hline Gibt es im WC - Raum ein Waschbecken? [Ex] & JA \\
\hline Ist das WC-Papier vom WC aus sitzend erreichbar? [Ex] & JA \\
\hline $\begin{array}{l}\text { Wenn es Haltegriffe gibt: ist zumindest der Haltegriff auf der Anfahrseite aufklappbar? [No] } \\
\text { Anmerkung: beide aufklappbar }\end{array}$ & JA \\
\hline $\begin{array}{l}\text { Wenn es waagrechte Haltegriffe gibt: sind diese in einer Höhe von } 75-85 \mathrm{~cm} \text { montiert? (ÖNorm B } \\
\text { 1600: einreihig } 75 \mathrm{~cm} \text {, zweireihig max. } 85 \mathrm{~cm} \text { ) [Ex] } \\
\text { Anmerkung: } \mathrm{H}=83 \mathrm{~cm}\end{array}$ & JA \\
\hline
\end{tabular}

Abbildung 10: Bereit für Barrierefreiheit 2 (2017).

Wenn die genauen Maßangaben von Hoteleinrichtungen bekannt sind, kann jeder Mensch mit einer körperlichen Behinderung vor einer Reise bereits sagen, ob das Hotel oder die Freizeitaktivität für ihn geeignet sind. 


\subsubsection{Holidays on Wheels}

"Holidays on Wheels - Accessible Holidays" ist europaweit die einzige Anwendungssoftware (App), die barrierefreie Urlaubs- und Freizeitangebote unter Berücksichtigung von Qualitätskriterien darstellt. Die App soll Menschen mit Behinderung den Zugang zu barrierefreien Erlebnissen, spontanen Aktivitäten, kulinarischen Eindrücken oder adrenalinreichen Abenteuern erleichtern. Es handelt sich dabei um eine Sammlung barrierefreier Urlaubs- \& Freizeitmöglichkeiten. Es werden viele Bereiche der touristischen Servicekette von „Holidays on Wheels“ abgedeckt. Die Bereiche Informieren und Vorbereiten, Wohnen und Schlafen, Essen und Trinken, Freizeit und Sport werden hinsichtlich ihrer Barrierefreiheit geprüft und in ihrer Eignung für Rollstuhl, Rollator oder für Menschen mit Seh- oder Hörbeeinträchtigung kategorisiert. Es werden auf der Onlinekarte der App auch alle öffentlichen Eurokey-Toiletten angezeigt, damit es die Möglichkeit gibt, zu sehen wo in der Stadt die nächste barrierefreie Toilette aufzufinden ist (Fojan, 2018).

„Bereit für Barrierefreiheit“ und „Holidays on Wheels“ bieten ein breites Spektrum an barrierefreien Angeboten. „Holidays on Wheels“ ist einfach am Smartphone anzuwenden und „Bereit für Barrierefreiheit“ bietet genaue Vermessungsdaten für barrierefreies Reisen.

Praktische Realisierungsmöglichkeiten zeigen die Bemühungen, die es bereits im barrierefreien Tourismus gibt. Trotz der verschiedenen Möglichkeiten, die für Menschen mit einer körperlichen Behinderung bestehen, kommt es zu Problemen auf Reisen. Diese werden im nächsten Kapitel statistisch erfasst. Des Weiteren werden die Hypothesen in Bezug auf barrierefreien Tourismus erläutert, ausgewertet und interpretiert. 


\section{Empirischer Teil}

Der empirische Teil dieser Arbeit befasst sich mit der Untersuchung zu barrierefreiem Tourismus in Österreich. Ziel dieser Studie ist es, einen Überblick über Probleme und Möglichkeiten barrierefreien Reisens zu erarbeiten, um zukünftige Planungen zu erleichtern. Im ersten Abschnitt werden die Forschungsfragen präzisiert und davon ausgehend die zugehörigen Hypothesen beschrieben. Anschließend werden die verwendeten Erhebungsinstrumente vorgestellt. Das Untersuchungsdesign wird mit den Methoden der Fragebogengestaltung und den Methoden der Datenerhebung vorgestellt. Die Stichprobe wird beschrieben. Daraufhin werden die methodenbezogenen und die weiteren relevanten Ergebnisse der Arbeit angeführt. Zuerst werden die Hypothesen und zentrale Fragestellungen der Studie angeführt.

\section{Hypothesen und zentrale Fragestellungen}

Welche Barrieren und Grenzen treten am häufigsten im Zuge der touristischen Servicekette auf?

Hypothese 1 und 2 beziehen sich auf diese Fragestellung. Die dafür verwendeten Fragen befinden sich im Fragebogen, der im Anhang angeführt wird.

\section{Hypothese 1}

H1: Es bestehen Unterschiede bezüglich des Auftretens von Problemen in den Bereichen Unterkunft, An- und Abreise, Freizeitangebot und Planung.

H0: Es bestehen keine Unterschiede bezüglich des Auftretens von Problemen in den Bereichen Unterkunft, An- und Abreise, Freizeitangebot und Planung.

Die erste Hypothese bezieht sich auf das Auftreten von Problemen entlang der touristischen Servicekette. In der Studie von Flucher, Parigger \& Zirngast (2013) kam es zu Unterschieden in der Problemanzahl bei den Bereichen der touristischen Servicekette. In der Hypothese 1 wird die Vermutung angestellt, dass sich die unterschiedlichen Bereiche hinsichtlich des Problemaufkommens unterscheiden. Diese Hypothese dient dazu einen Überblick zu verschaffen, wo die meisten Probleme auftreten. 
In der zweiten Hypothese wird danach aufgezeigt, wie störend die Probleme in den unterschiedlichen Bereichen empfunden werden.

\section{Hypothese 2}

H1: Es bestehen unterschiedliche Bewertungen bezüglich des Problemempfindens, der Bereiche Unterkunft, An- und Abreise, Freizeitangebot und Planung.

H0: Es bestehen keine unterschiedlichen Bewertungen bezüglich des Problemempfindens der Bereiche Unterkunft, An- und Abreise, Freizeitangebot und Planung.

Bei den Studien von Flucher, Parigger \& Zirngast (2013) und von Ranneger (2006) wurden identische Skalen verwendet, die das Problemauftreten und das Problemempfinden auf Reisen erfragen. Passende Fragen dieser Skalen wurden auch für diese Studie herangezogen. Um die Berechnung von Mittelwertsvergleichen zu erleichtern, wurde jede Skala auf die fünf häufigsten Probleme gekürzt. Ein weiterer Vorteil dieses Vorgehens ist, dass die Ergebnisse aus vorherigen Studien genützt werden und die häufigsten Probleme nun miteinander verglichen werden können. Dadurch wird sichtbar, welcher der verschiedenen Bereiche der ist, der das höchste Problemempfinden aufweist. Es soll mittels Varianzanalyse berechnet werden, ob signifikante Unterschiede zwischen den unterschiedlichen Bereichen der touristischen Servicekette bestehen.

\section{Fragestellung 2}

Welche Informationen sind für Reisende mit körperlicher Beeinträchtigung besonders wichtig?

\section{Hypothese 3}

H1: Die Wichtigkeit der Informationen bezüglich Barrierefreiheit von Unterkunft, An- und Abreise, Freizeitangebot und Planung wird unterschiedlich bewertet.

H0: Die Wichtigkeit der Informationen bezüglich Barrierefreiheit von Unterkunft, An- und Abreise, Freizeitangebot und Planung wird nicht unterschiedlich bewertet. In dieser Hypothese soll aufgezeigt werden, ob unterschiedliche Bewertungen der Wichtigkeit der Informationen bestehen. Informationen zu den unterschiedlichen Bereichen der touristischen Servicekette ermöglichen häufig erst eine barrierefreie Reise. 
Das Aufzeigen der Unterschiedlichkeit der Relevanz von Informationen kann Reiseanbietern dabei behilflich sein, wichtige Informationen zu unterschiedlichen Bereichen besonders zu betrachten, da nach Wilken (2016) Reisebüros mit der Verwirklichung von barrierefreien Angeboten oft überfordert sind. Es soll eine Hilfe für zukünftige Planungen darstellen, da die wichtigsten Informationen zuerst genannt werden können.

Welche Möglichkeiten zur Realisierung einer Reise werden von Menschen mit Behinderung genutzt?

\section{Hypothese 4}

H1: Menschen mit Körperbehinderung buchen ihren Urlaub häufiger privat als über sonstige Wege.

H0: Menschen mit Körperbehinderung buchen ihren Urlaub nicht häufiger privat als über sonstige Wege.

Bereits bei Flucher, Parigger \& Zirngast (2013), die eine Befragung zu barrierefreiem Tourismus in Österreich durchgeführt haben, werden Urlaube häufiger privat organisiert als über Reisebüros, Spezialreisebüros oder Vereine.

Wilken (2016) beschreibt ein Fehlen von Angeboten und stark begrenzten Zugang im barrierefreien Tourismus. Es gilt noch immer als ein randständiges Marktsegment. Spezialreisekataloge für behinderte Urlauber sind in Reisebüros nur selten vorrätig. Wilken sieht die Zurückhaltung im Tourismus auch aufgrund der Heterogenität der Zielgruppe, da Behinderungen vielfältig auftreten können und absolute Ansprüche, wie „barrierefreier Tourismus für alle“ die Anbieter überfordern. Des Weiteren können auch Kosten für barrierefreie Testungen und den Erhalt von kennzeichnenden Piktogrammen entstehen. Das sind nach Wilken die Hauptgründe, warum das große Marktsegment des barrierefreien Tourismus von Reisebüros nicht ausgeschöpft wird, denn 48 Prozent der Menschen mit Beeinträchtigung würden häufiger verreisen, wenn es zusätzliche barrierefreie Angebote gäbe (Wilken, 2016).

Aus den fehlenden Angeboten für Menschen mit Behinderung und den Ergebnissen der Studie von Flucher, Parigger \& Zirngast (2013) kann geschlossen werden, dass Menschen mit Körperbehinderung ihren Urlaub häufiger privat buchen als über sonstige Wege. 


\section{Hypothese 5}

H1: Das Internet wird am häufigsten als wichtige Informationsquelle, für die Planung des letzten barrierefreien Urlaubs genannt.

H0: Das Internet wird nicht am häufigsten als wichtige Informationsquelle, für die Planung des letzten barrierefreien Urlaubs genannt.

In der Studie von Laburda \& Smikac (2009) über grundlegende Aspekte der Urlaubsplanung wurden Reisepläne und Aktivitäten für einen Urlaub ohne Barrieren evaluiert. Es wurden 126 Personen mit körperlicher Behinderung befragt, welche Quellen sie für ihre Reisevorbereitung nutzen. 9 Prozent nannten Bekannte, 7 Prozent nannten Broschüren von Reiseveranstaltern und 69 Prozent der Befragten gaben das Internet als wichtigste Quelle für die Reisevorbereitung an. Daher wird davon ausgegangen, dass das Internet die wichtigste Informationsquelle bei der Planung eines barrierefreien Urlaubs darstellt.

\section{Hypothese 6}

\section{H1: Es nehmen mehr Frauen Reiseassistenz in Anspruch als Männer.}

H0: Es nehmen nicht mehr Frauen Reiseassistenz in Anspruch als Männer.

Hierbei handelt es sich um eine explorative Hypothese, da sich in der behandelten Fachliteratur keine Belege dafür finden lassen.

Der Verein ISI (Initiative Soziale Integration) aus Graz bietet Assistenz für das Studium, für Arbeit und Dienstreisen an. Der hohe Frauenanteil der AssistenznehmerInnen wurde im Team schon öfter beobachtet. Der momentane Stand (02.05.2018) liegt bei 43 Frauen und 30 Männern. Daraus entstand das Interesse zu testen, ob auch mehr Frauen als Männer Assistenz bei Urlaubsreisen in Anspruch nehmen. Interessant ist hier zu sehen, ob dieser Unterschied nur bei ISI oder auch bei der vorliegenden Befragung auftritt. 


\section{Untersuchungsdesign}

Die Befragung wurde mit einem Online-Fragebogen durchgeführt (siehe Anhang). Die Fragebogenerstellung erfolgte in Anlehnung an den Fragebogen von Rannegger (2006) und Flucher et.al. (2013). Um die Verständlichkeit für die Zielgruppe des Fragebogens zu gewährleisten, haben ihn fünf Personen mit körperlicher Behinderung überprüft. Es wurden mit allen Personen Gespräche über die einzelnen Fragen geführt, um festzustellen, ob die Fragen eindeutig, klar und verständlich formuliert sind. Des Weiteren wurde geklärt, ob alle Begriffe und Bezeichnungen auch so von Menschen mit körperlicher Behinderung selbst verwendet und akzeptiert werden.

Die Übertragung des Fragebogens zu einer Online-Variante erfolgte über das Programm „Lime-Survey“. Der generierte Link, über den der Fragebogen geöffnet werden kann, wurde in Folge an verschiedene Vereine und Universitäten versendet, die ihn wiederum auf ihren Seiten und über ihre Mailverteiler verbreitet haben. Diese Institutionen waren:

- Zentrum integriert Studieren der Universität Graz

- Universität Wien

- Liste von Rollstuhltanz-Sportvereinen

- Multiple Sklerose Gesellschaft

- Lebenshilfe (österreichweit)

- Mosaik

- Diakonie

- ISI (Initiative Soziale Integration)

- „Holidays on Wheels“

- ,atempo“

- Selbstvertretungsvereine (Selbstbestimmt Leben)

- Universitäten (Universitäten Österreichweit, mit jeweiligen Inklusionsbüros: In Graz beispielsweise Selbstbestimmt Leben)

- Behindertensportvereine (alle Sportvereine außer Blindensportverein und Gehörlosenverband) 
Die Methode der Online-Befragung ist kritisch zu betrachten. Auch wenn die Zielgruppe in der Einleitung der Befragung beschrieben wird, kann nicht klar verfolgt werden, wer teilnimmt und wie oft eine einzelne Person die Befragung ausfüllt. Der Rücklauf war nach der Kontaktherstellung mit den Rollstuhlsportvereinen mit Abstand am höchsten. Daraus kann geschlossen werden, dass die TeilnehmerInnen der Umfrage eher sportlich und aktiv sind, was einen Einfluss auf die Ergebnisse haben kann.

\subsection{Fragebogengestaltung}

In diesem Kapitel werden die verschiedenen Bereiche des Fragebogens genauer erörtert. Der Fragebogen ist angelehnt an die Einteilung der touristischen Servicekette. Er ist teilweise von Rannegger (2006) und Flucher et al. (2013) übernommen. Diese Teile werden genau beschrieben. Der gesamte Fragebogen wird im Anhang angeführt.

\section{Reiseassistenz}

Die ersten drei Fragen beinhalten das Thema Reiseassistenz. Es wird beispielsweise nach der Inanspruchnahme von Reiseassistenz gefragt und wie diese organisiert wird. Die Fragen zur Reiseassistenz dienen zur Beantwortung der Hypothese 6: „Es nehmen mehr Frauen Reiseassistenz in Anspruch als Männer.“

\section{Buchung und Reiseplanung}

Die darauffolgenden Fragen 4-7 betreffen die Art der Buchung und Reiseplanung. Sie zielen auf die Beantwortung der 4. Hypothese ab:

„Menschen mit Körperbehinderung buchen ihren Urlaub häufiger privat als über sonstige Wege.“ Die dazugehörige Frage lautet: Wie haben sie ihren letzten Urlaub gebucht? Die Antwortmöglichkeiten hierfür sind: Privat, Privat und Reisebüro, Reisebüro, Freunde und Familie, Spezialreisebüros/-Anbieter oder Rollstuhl/-Behindertenverein.

Die nächsten Fragen zielen darauf ab, herauszufinden, welche Relevanz das Internet als Informationsquelle für Reisen hat und sollen zur Beantwortung der Hypothese 5 dienen: „Das Internet wird am häufigsten als wichtigste Informationsquelle genannt, für die Planung des letzten barrierefreien Urlaubs.“ Es ist unter 10 verschiedenen Antwortmöglichkeiten zu wählen und es ist als Single Choice zu beantworten, da es hierbei nur um die wichtigste Informationsquelle geht. 


\section{Planung}

Die Bereiche Planung, An- und Abreise, Unterkunft und Freizeitangebot wurden vom Fragebogen von Flucher at al. (2013) übernommen. Es wird mit diesen Fragen erhoben, welche Probleme am häufigsten in der touristischen Servicekette auftreten und wie sehr diese stören. Es wurden hierbei die Ergebnisse der Studie genutzt und es wurden jeweils die 5 Probleme vorgegeben, die am häufigsten genannt worden waren, um weniger relevante Probleme bereits vor der Befragung auszuschließen und zusätzlich die Berechnung eines Mittelwertvergleichs zu erleichtern.

Beispiel: Fehlende, zu ungenaue oder falsche Informationen vom Reisebüro über die $\mathrm{Zu}-$ gänglichkeit/Barrierefreiheit am Urlaubsort. Die Fragen sind jeweils mit ja und nein und mit der jeweiligen Gewichtung des Störfaktors zu beantworten.

\section{An- und Abreise}

Hierbei soll beantwortet werden, mit welchen Problemen die ProbandInnen konfrontiert waren und als wie störend sie diese hinsichtlich ihrer An- und Abreise empfunden haben. Beispielsweise wurde hier gefragt, ob sanitäre Einrichtungen im Zug/Bus während des letzten Urlaubs benutzbar waren.

\section{Unterkunft}

Dieser Bereich untersucht Probleme bezüglich der Unterkunft. Beispielsweise wird hier nach baulichen Barrieren oder eingeschränkten Bewegungsflächen in den Zimmern gefragt.

\section{Freizeitangebot}

Dieser Themenschwerpunkt zielt darauf ab, das Problemauftreten und -empfinden bezüglich des Freizeitangebots zu erfragen. Zum Beispiel wird nach der Nutzbarkeit von sanitären Einrichtungen am Ausflugsziel gefragt oder ob eine Möglichkeit bestand, überhaupt an Freizeitangeboten teilnehmen zu können. 


\section{Information}

Die Fragen 28-36 betreffen die Informationsbeschaffung und gehen wiederum auf die Bereiche Planung, An- und Abreise, Unterkunft und Freizeitangebot ein. Es wird nach Informationsportalen gefragt, die zur barrierefreien Urlaubsverwirklichung angewendet werden. Diese Fragen dienen zur Beantwortung der Hypothese 3: „Die Wichtigkeit der Informationen bezüglich Barrierefreiheit von Unterkunft, An- und Abreise, Freizeitangebot und Planung wird unterschiedlich bewertet.“

\section{Allgemeine Informationen}

Die letzten vier Fragen beinhalten allgemeine Informationen zu den ProbandInnen. Hier wird nach Geschlecht, Alter, Bundesland und Unterstützungsmittel gefragt. Diese Fragen dienen teilweise auch zur Beantwortung der 6. Hypothese: „Es nehmen mehr Frauen Reiseassistenz in Anspruch als Männer.“

\subsection{Stichprobenbeschreibung}

Dieses Kapitel widmet sich der Beschreibung der Stichprobe. Es werden deskriptivstatistische Ergebnisse angegeben und grafisch dargestellt.

Was für einen Menschen mit einer Gehbehinderung eine Barriere ist, kann für einen blinden Menschen zur Orientierung dienen (Kastl, 2017). Aufgrund dieser unterschiedlichen Anforderungen wurden in dieser Studie ausschließlich Menschen mit einer, den Bewegungsapparat betreffenden, körperlichen Behinderung befragt. Um dies zu gewährleisten, wird im Fragebogen nach Hilfsmitteln gefragt und es werden speziell Vereine angeschrieben, die größtenteils auf Menschen mit einer Behinderung des Bewegungsapparates ausgerichtet sind. Des Weiteren wird eine Zielgruppenbeschreibung vor der Befragung angeführt, um den ProbandInnen zu zeigen, ob sie für die Studie geeignet sind.

Weitere Personen, die in ihrer Mobilität eingeschränkt sind, können in dieser Arbeit nicht berücksichtigt werden, da die Ansprüche von Barrieren im Tourismus zu sehr variieren. $\mathrm{Zu}$ Menschen mit Mobilitätseinschränkung zählen beispielsweise noch Eltern mit Kinderwagen, stark übergewichtige Personen oder Menschen mit einer Sehbehinderung (Leyendecker, 2005). 
Es werden nur erwachsene Personen ab 18 Jahren befragt, da eine höhere Wahrscheinlichkeit besteht, dass im Gegensatz zu Kindern und Jugendlichen die Reiseplanung selbstständig durchgeführt wird.

Die Grundgesamtheit bilden Menschen mit körperlicher Behinderung ab 18 Jahren aus Österreich. Was unter körperlicher Behinderung verstanden wird, ist im Kapitel 2 beschrieben. An der Umfrage haben insgesamt 111 Menschen teilgenommen. Die Teilnahme der ProbandInnen beruhte auf Freiwilligkeit und Anonymität. Die Erhebung fand im Zeitraum von Juli bis Dezember 2017 statt. Weitere Angaben über die ProbandInnen werden im nächsten Kapitel beschrieben.

\subsection{Soziodemographische Analyse}

Die soziodemografische Analyse umfasst Geschlecht, Alter und Herkunft. Diese Daten werden beschrieben, grafisch aufgezeigt und die Verteilung der Mittelwerte wird angeführt.

\section{Geschlecht der Befragten}

Von den 111 TeilnehmerInnen sind 55 Prozent (61Peronen) männlich und 41,4 Prozent (46 Personen) weiblich. Weitere 4 Personen haben keine Antwort auf die Frage gegeben.

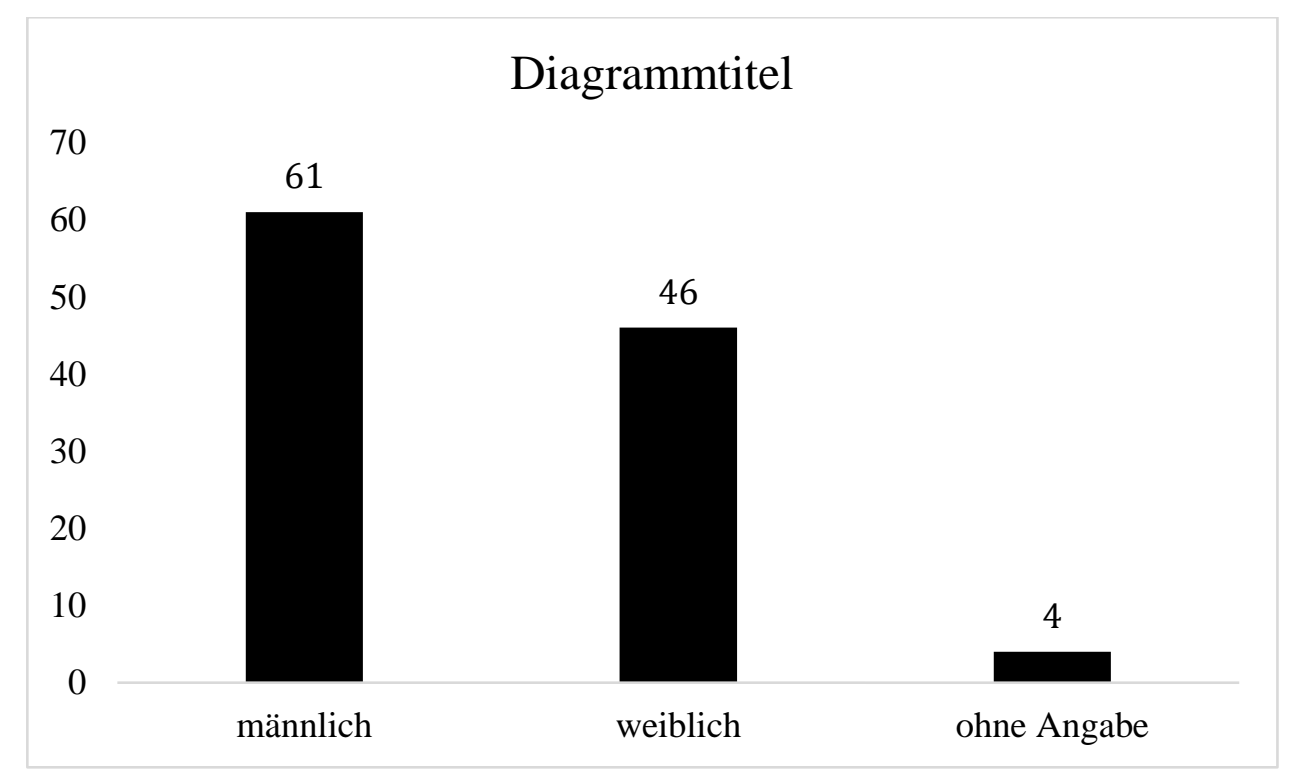

Abbildung 11: Geschlechterverteilung der Stichprobe in Prozentangabe 


\section{Altersstruktur der ProbandInnen}

In Bezug auf das Alter bilden die größten Gruppen, die der 31 bis 40-Jährigen und die 51 bis 60-Jährigen. Die kleinsten Gruppen sind die 61 bis 70-Jährigen (11 Personen) und die

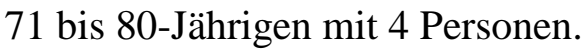

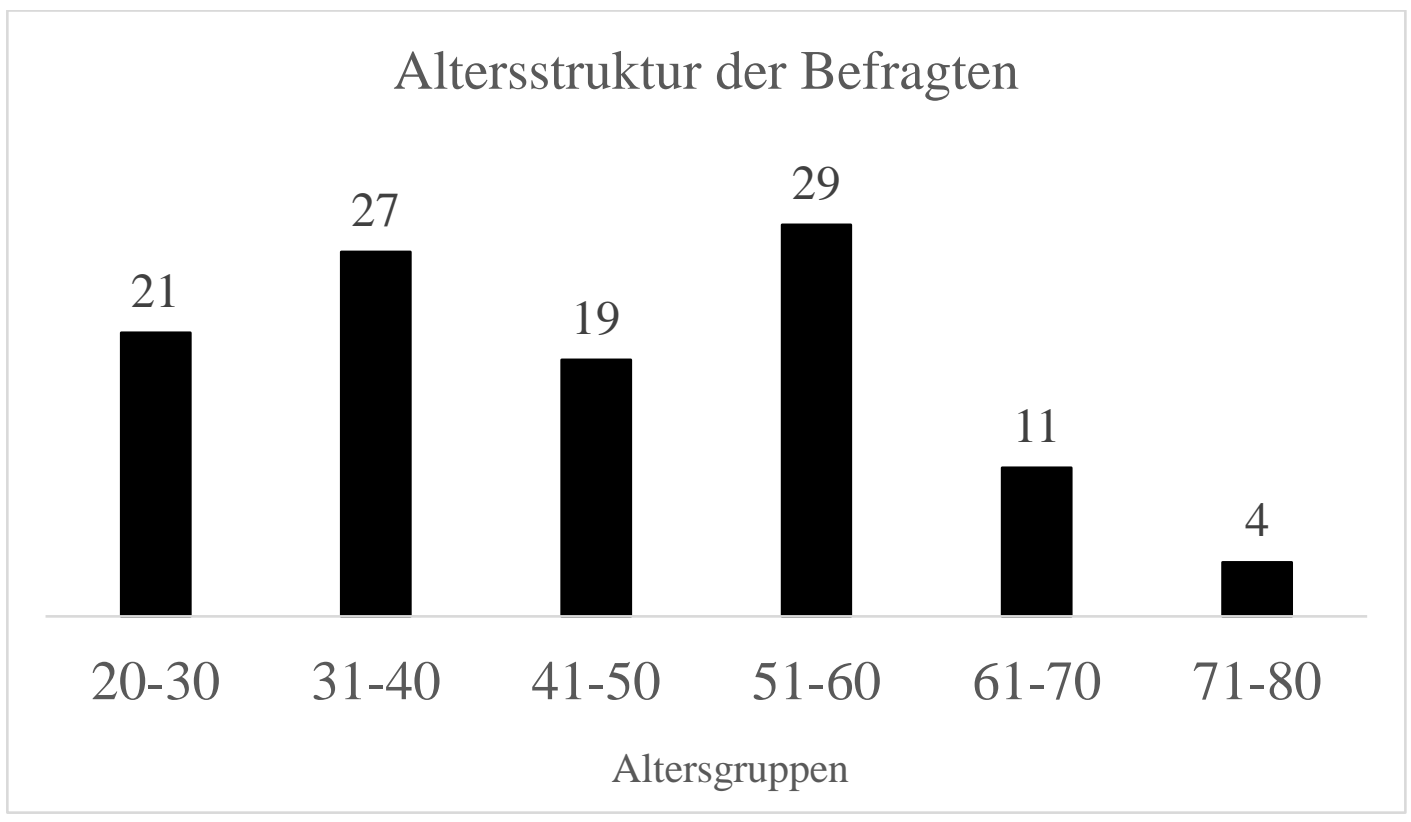

Abbildung 12: Altersstruktur der Befragten 


\section{Herkunft der TeilnehmerInnen}

Es haben Menschen mit einer körperlichen Behinderung aus allen 9 Bundesländern an der Umfrage teilgenommen. Die meisten ProbandInnen kommen aus der Steiermark (36). Es hat jedoch nur eine Person aus Kärnten teilgenommen.

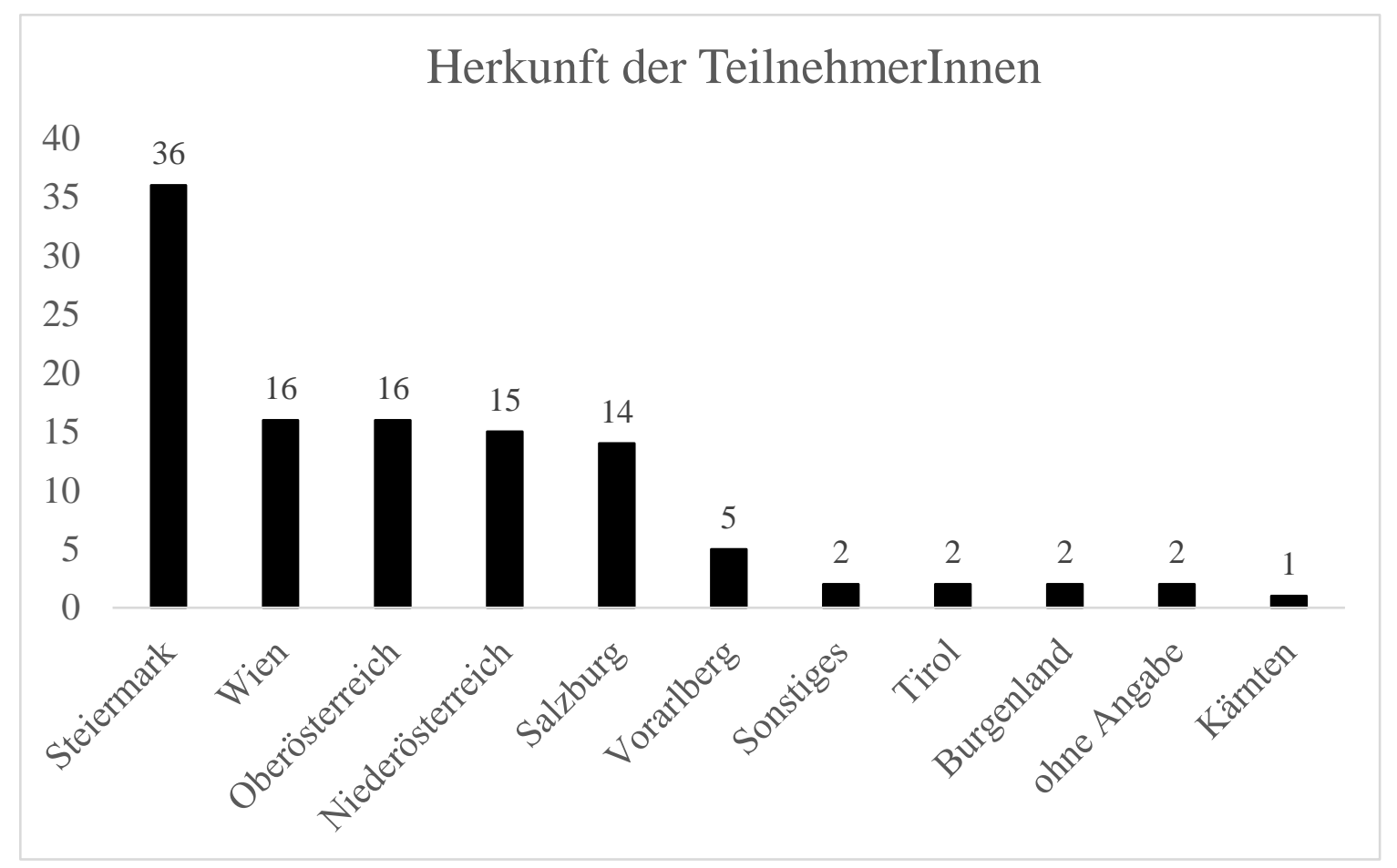

Abbildung 13: Herkunft der TeilnehmerInnen 


\section{Unterstützungsmittel}

Die nächste Grafik soll veranschaulichen auf welche Unterstützungsmittel von den TeilnehmerInnen verwendet werden. Es waren Mehrfachnennungen möglich.

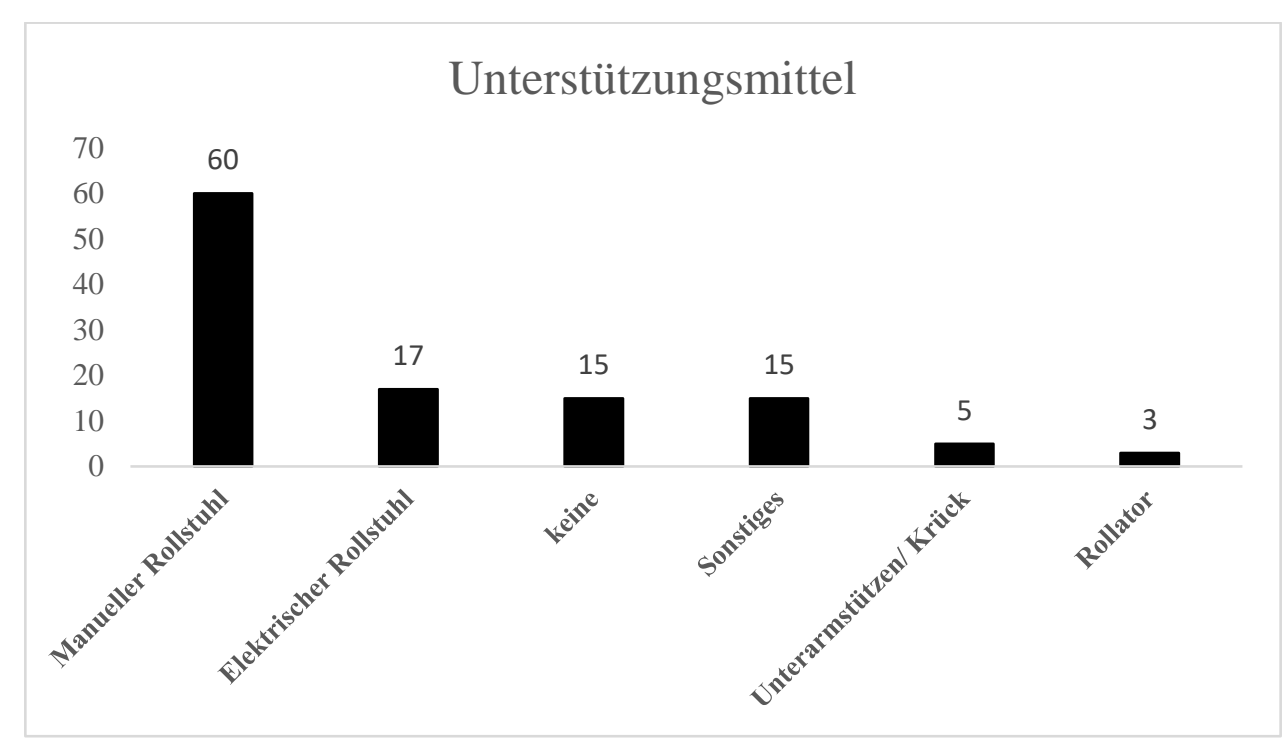

Abbildung 14: Unterstützungsmittel

\section{Methoden der statistischen Analyse}

Die Methoden der statistischen Analyse werden hier in der Reihenfolge vorgestellt, wie sie auch in Folge im nächsten Kapitel vorkommen. Die Methoden dienen zur Überprüfung der Hypothesen, die im 6. Kapitel vorgestellt werden.

\section{Einfaktorielle Varianzanalyse mit Messwiederholung}

Die einfaktorielle Varianzanalyse mit Messwiederholung untersucht die Frage, ob sich die Ausprägung eines Merkmals von abhängigen Stichproben zu verschiedenen Messzeitpunkten unterscheidet. Es wird daher getestet, ob sich die Mittelwerte mehrerer abhängiger Stichproben unterscheiden (Rasch, Friese, Hofmann \& Naumann, 2010).

Die Stichprobe ist in dieser Arbeit abhängig, da eine Messwiederholung durchgeführt wird. Die Messwerte stammen von den gleichen Personen zu unterschiedlichen Zeitpunkten (Themenbereichen). 
Die Varianzanalyse prüft, ob sich die Mittelwerte zu den Messzeitpunkten signifikant unterscheiden. Die Messzeitpunkte werden als Faktorenstufen bezeichnet, da es im Fall dieser Arbeit nicht um die Entwicklung über die Zeit geht, sondern das Ziel ist der Vergleich der Messungen zu verschiedenen Themenbereichen.

Voraussetzung ist, dass die abhängige Variable intervallskaliert und normalverteilt ist. Eine weitere Voraussetzung ist eine gegebene Sphärizität. Diese wird über den MauchlyTest geprüft. Ist dieser nicht signifikant, kann von Sphärizität ausgegangen werden. Bei einem nicht signifikanten Testergebnis des Mauchly-Test, wird der Wert der angenommenen Sphärizität verwendet. Ist der Mauchly-Test auf Sphärizität signifikant, wird das Greenhouse-Geisser-Korrekturverfahren angewendet. Dieser Test ist ab drei Messzeitpunkten relevant, da es sonst nur ein Paar zu vergleichen gibt. Es wird bei allen Varianzanalysen dieser Arbeit eine Bonferroni Korrektur durchgeführt. Dabei wird das gewünschte Gesamt-Signifikanzniveau durch die Anzahl benötigter Einzeltests dividiert. Das Ergebnis ist das Signifikanzniveau für jeden Einzelvergleich (Rasch, Friese, Hofmann \& Naumann, 2010).

In dieser Arbeit werden die Vergleiche der Mittelwerte der Problembereiche (An- und Abfahrt, Reiseplanung, Unterkunft und Freizeitangebot) paarweise durchgeführt.

\section{Eindimensionaler Chi-Quadrat-Test}

Dieser Test wird dann herangezogen, wenn die Versuchspersonen einer Population anhand eines Merkmals mit zwei oder mehr Stufen klassifiziert werden. Unter der Gleichverteilungsannahme sollten die Häufigkeiten über alle Stufen des Merkmals hinweg gleich sein. Es werden die beobachteten Häufigkeiten den erwarteten Häufigkeiten gegenübergestellt (Rasch, Friese, Hofmann \& Naumann, 2010). 


\section{Ergebnisse der Hypothesentests}

In diesem Kapitel werden die angeführten Hypothesen mittels statistischer Verfahren überprüft. Um die Nachvollziehbarkeit der Ergebnisse zu gewährleisten, werden die Verfahren beschrieben und im Anhang werden relevante Tabellen der Tests angeführt.

\subsection{Welche Barrieren und Grenzen treten am häufigsten im Zuge der touristischen Servicekette auf?}

Die ersten beiden Varianzanalysen zielen auf die Beantwortung der ersten Fragestellung ab. Zuerst wird abgeklärt, welche Probleme auf der touristischen Servicekette vorkommen. Die zweite Hypothese bestimmt, wie störend die Probleme in den unterschiedlichen Bereichen wahrgenommen werden.

\subsubsection{Auftreten von Problemen auf der touristischen Servicekette}

In diesem Kapitel werden die ersten beiden Hypothesen überprüft und es werden Varianzanalysen mit Messwiederholung durchgeführt. Hypothese eins testet das Aufkommen von Problemen auf der touristischen Servicekette. Die Faktorstufen sind gereiht nach dem Fragebogen und nach der organisatorischen Reihung eines Reiseverlaufs. Diese sind Planung, An- und Abreise, Unterkunft und Freizeitangebot. Dieselbe Reihung wird auch bei den folgenden Varianzanalysen vorgenommen und wird auch bei den Tabellen im Anhang beibehalten. In der ersten Hypothese wird davon ausgegangen, dass es Unterschiede bezüglich des Auftretens von Problemen der Bereiche Planung, An- und Abreise, Unterkunft und Freizeitangebot gibt.

UV: Problemfelder

AV: Problemaufkommen 
Zur Berechnung werden hier die Fragestellungen zum Problemaufkommen herangezogen. Es gibt die Möglichkeit zwischen Ja und Nein zu wählen und daraus entsteht der Summenscore, der zwischen den Grenzen von 0-5 Punkten variieren kann. Es werden paarweise Vergleiche der Faktorenstufen (Planung, An- und Abreise, Unterkunft und Freizeitangebot) durchgeführt. Das Ergebnis des Mauchly-Tests auf Sphärizität befindet sich innerhalb des Signifikanzniveaus $(p=.01)$, daher wird das Greenhouse-Geisser Korrekturverfahren angewendet.

Die Analyse ergab, dass zwischen den Problemfeldern Unterschiede hinsichtlich des Problemaufkommens bestehen $(p<.001)$. Damit wurde Hypothese 1 bestätigt: Es bestehen Unterschiede bezüglich des Auftretens von Problemen in den Bereichen Unterkunft, An- und Abreise, Freizeitangebot und Planung. Paarweise Vergleiche haben ergeben, dass es zwischen Planung und Freizeitangebot sowie bei An- und Abreise und Freizeitangebote signifikante Unterschiede hinsichtlich des Problemempfindens gibt Die deskriptive Statistik und die paarweisen Vergleiche deuten darauf hin, dass Probleme hinsichtlich des Freizeitangebots am häufigsten wahrgenommen werden.

Abbildung 15 veranschaulicht die unterschiedlichen Problembewertungen und zeigt die Mittelwerte samt Standardfehler an. Die Reihung der Problemaufkommen wird nach einem Reiseverlauf angezeigt. Die deskriptive Statistik und die paarweisen Vergleiche zeigen, dass die meisten Probleme in Verbindung mit dem Freizeitangeboten auftreten. Die paarweisen Vergleiche zeigen signifikante Unterschiede zwischen den Bereichen Planung und Freizeitangebote, des Weiteren werden auch signifikante Unterschiede zwischen An- und Abreise und Freizeitangebote festgestellt. 


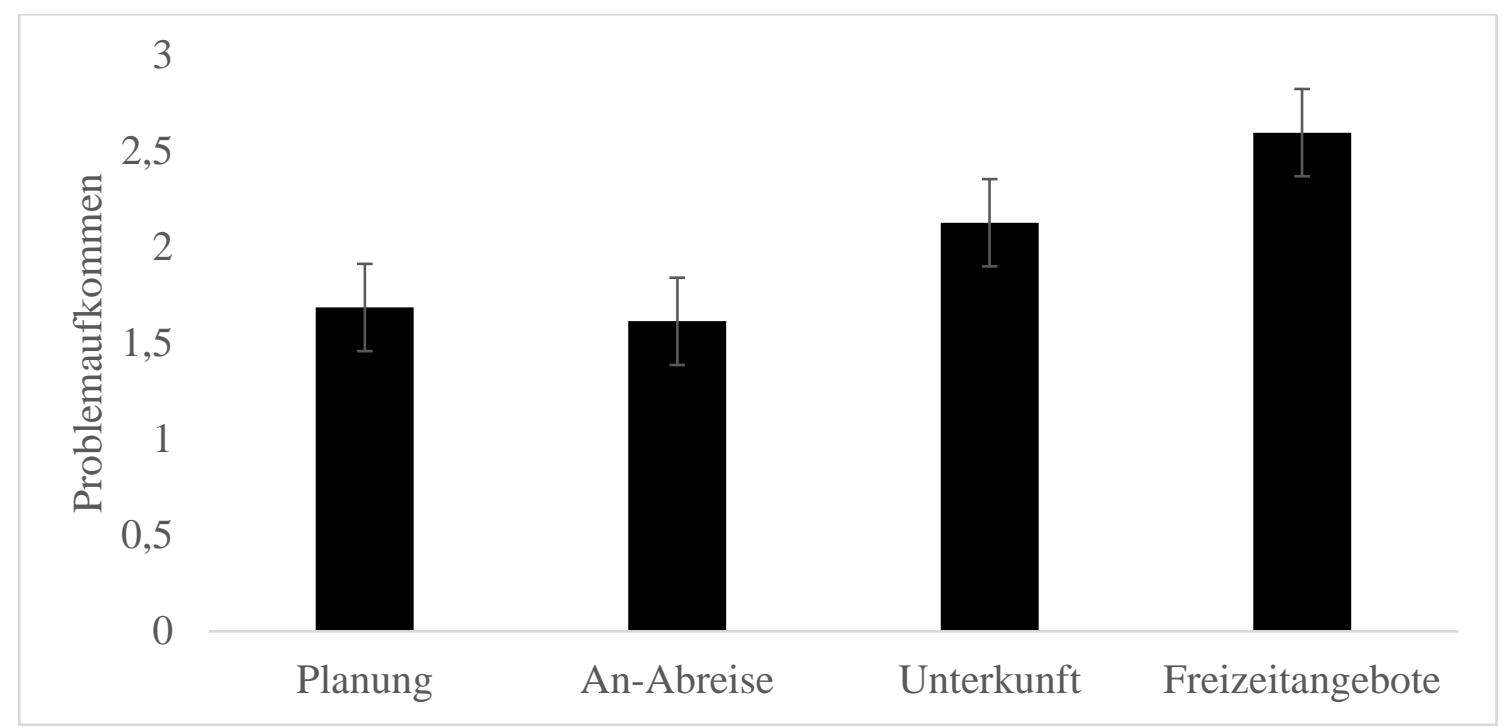

Abbildung 15: Problemaufkommen in den Bereichen: Planung, An- und Abreise, Unterkunft und Freizeitangebote.

\subsubsection{Problemempfinden auf der touristischen Servicekette}

Zur Berechnung werden hier dieselben Fragestellungen wie im Kapitel zuvor aufgegriffen, nur kommt es hier nicht auf das Auftreten des Problems, sondern auf das Problemempfinden an. Es geht hier um die Frage, wie störend ein Problem ist und der Summenscore wurde folgendermaßen gebildet: es gab kein Problem (0), es gab ein Problem, aber es hat mich nicht gestört (1), es hat mich wenig gestört (2), es hat mich eher gestört (3) und es hat mich sehr gestört (4). Diese Varianzanalyse bestimmt, wie störend Probleme in den unterschiedlichen Bereichen von den ProbandInnen empfunden wurden und beantwortet damit die 2. Hypothese.

UV: Problemfelder

AV: Problemempfinden

Es wird eine einfaktorielle Varianzanalyse mit Messwiederholung durchgeführt, da dieselben ProbandInnen jeweils die unterschiedlichen Problemfelder bewerten. Da der $p$ Wert des Mauchly-Tests $=.01$ ist, wird das Greenhouse-Geisser Korrekturverfahren angewendet. Als Post Hoc Test werden paarweise Vergleiche der Faktorstufen (Planung, An- und Abreise, Unterkunft und Freizeitangebot) durchgeführt. 
Die Analyse ergab, dass es zwischen den verschiedenen Problemfeldern Unterschiede in ihrer wahrgenommenen Intensität gibt $(p<.001)$. Damit wird die Hypothese 2 bestätigt: Es bestehen unterschiedliche Bewertungen bezüglich des Problemempfindens der Bereiche Unterkunft, An- und Abreise, Freizeitangebot und Planung.

Abbildung 16 veranschaulicht die unterschiedlichen Problembewertungen. Paarweise Vergleiche haben ergeben, dass es zwischen An- und Abreise und Freizeitangebote einen signifikanten Unterschied hinsichtlich des Problemempfindens gibt $(p<.001)$ auch zwischen Freizeitangebote und Planung besteht ein signifikanter Unterschied $(p<.001)$. Die deskriptive Statistik und die paarweisen Vergleiche deuten darauf hin, dass Probleme hinsichtlich des Freizeitangebots als störender wahrgenommen werden als Probleme in den anderen Themenbereichen.

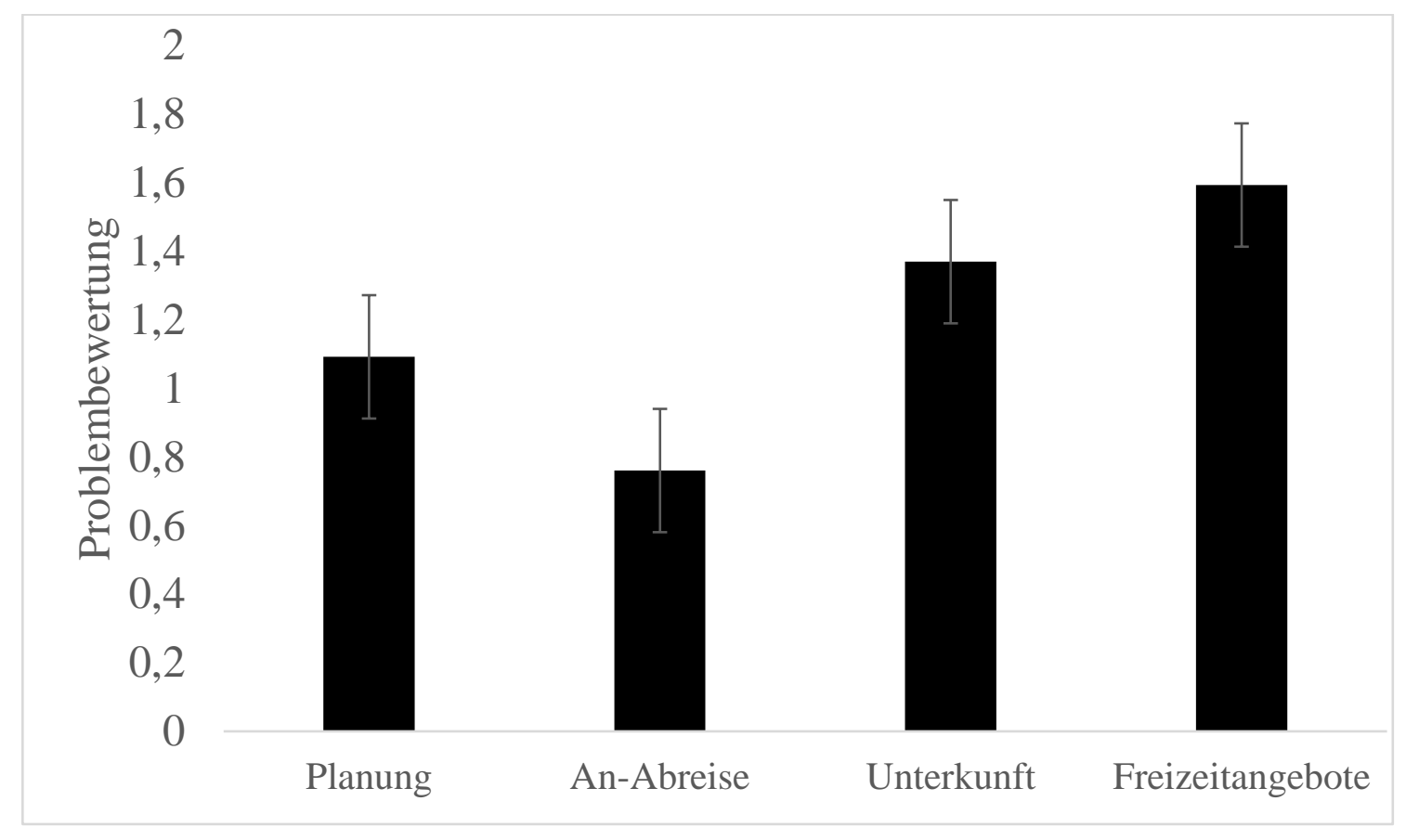

Abbildung 16: Problembewertung der Felder Planung, An- und Abreise, Unterkunft und Freizeitangebote.

Die häufigsten Barrieren auf der touristischen Servicekette treten im Zusammenhang mit dem Freizeitangebot auf. Reiseprobleme hinsichtlich des Freizeitangebots werden von den ProbandInnen auch am störendsten wahrgenommen. Die nächste Fragestellung beschäftigt sich mit der Relevanz von Reiseinformationen für Menschen mit einer Behinderung. 


\subsection{Welche Informationen sind für Reisende mit körperlicher Beeinträchtigung besonders wichtig?}

\subsubsection{Relevanz von Informationen auf der touristischen Servicekette}

In diesem Kapitel wird die Relevanz der Informationen hinsichtlich der unterschiedlichen Bereiche Planung, An- und Abreise, Unterkunft und Freizeitangebot, angeführt. Es wird eine Varianzanalyse mit Messwiederholung durchgeführt und der Summenscore wurde folgendermaßen gebildet: sehr wichtig (4), eher wichtig (3), wenig wichtig (2) und nicht wichtig (1).

UV: Reisebereiche

AV: Informationsrelevanz

Der Mauchly Test weist keine Signifikanz auf $(p=.107)$ Es kann davon ausgegangen werden, dass die Sphärizität gegeben ist. Die Analyse ergab, dass Unterschiede bezüglich der Relevanz von Informationen auf der touristischen Servicekette bestehen ( $p=.005)$. Damit wurde die 3. Hypothese bestätigt: Die Wichtigkeit der Informationen bezüglich Barrierefreiheit von Unterkunft, An- und Abreise, Freizeitangebot und Planung wird unterschiedlich bewertet. Als Post Hoc Test werden paarweise Vergleiche der Faktorstufen durchgeführt. Paarweise vergleiche haben ergeben, dass es zwischen An- und Abreise und Unterkunft signifikante Unterschiede hinsichtlich der Relevanz von Informationen gibt $(p=.012)$. Die deskriptive Statistik und die paarweisen Vergleiche deuten darauf hin, dass Informationen zur An- und Abreise am wichtigsten sind. 


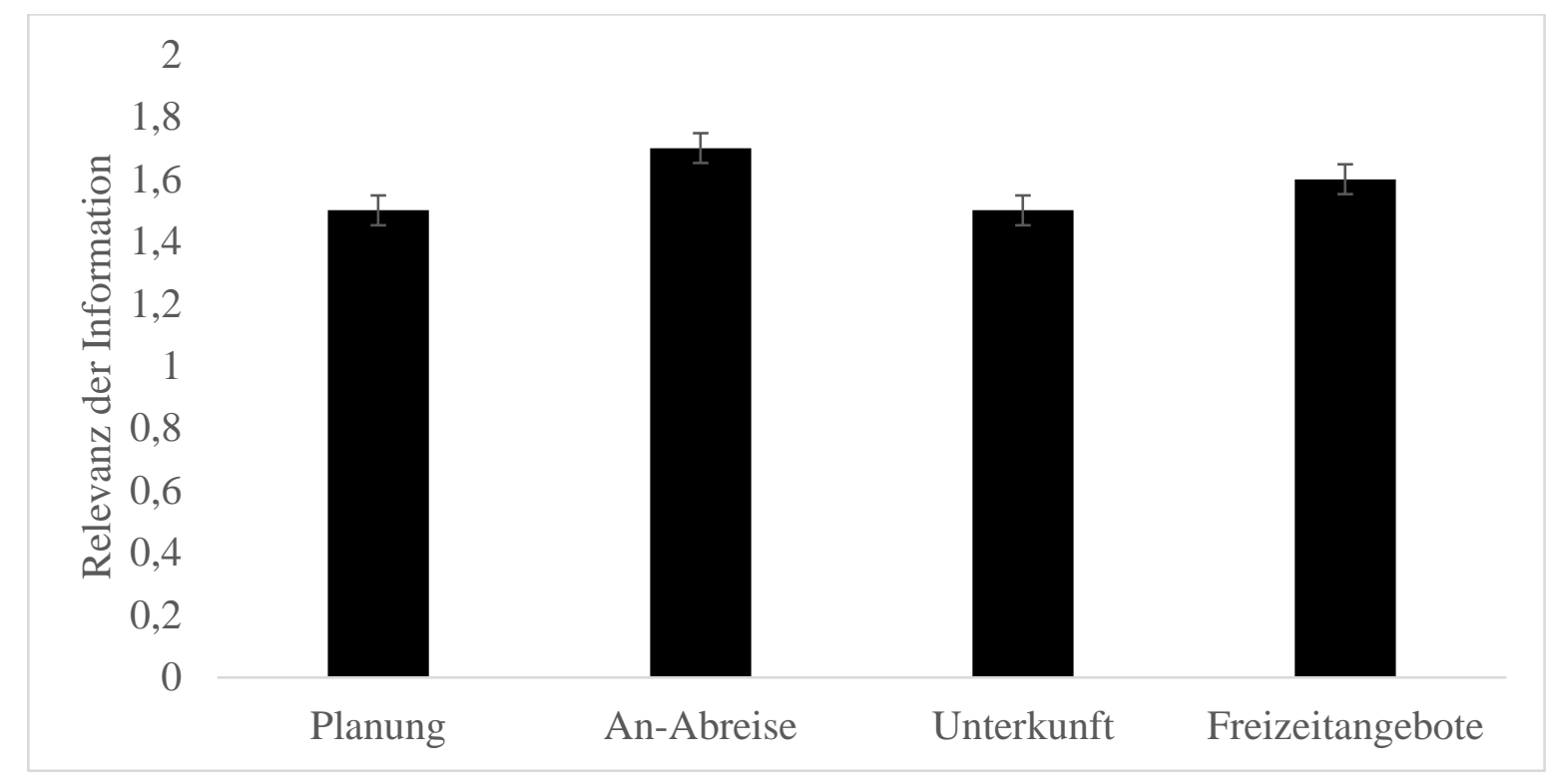

Abbildung 17: Relevanz der Informationen bezüglich Planung, An- und Abreise, Unterkunft und Freizeitangebote

Abbildung 17 veranschaulicht die unterschiedliche Relevanz von Informationen der Problembereiche. Die paarweisen Vergleiche zeigen einen signifikanten Unterschied zwischen den Bereichen An- und Abreise und Unterkunft $(p=.033)$. Die deskriptive Statistik und die paarweisen Vergleiche zeigen, dass der Bereich An- und Abreise am relevantesten hinsichtlich der Informationsbeschaffung ist. Im nächsten Kapitel werden Möglichkeiten zur Realisierung einer barrierefreien Reise angeführt.

\subsection{Welche Möglichkeiten zur Realisierung einer Reise werden von Menschen mit Behinderung genutzt?}

In diesem Kapitel geht es darum aufzuzeigen wie Menschen mit einer Körperbehinderung ihren Urlaub Buchen und Planen. Mit einem eindimensionalen Chi-Quadrat Test wird analysiert, ob die ProbandInnen unterschiedliche Buchungsarten nutzten. Die vorgegebenen Möglichkeiten waren Privat, Privat und Reisebüro, Reisebüro, Freunde und Familie, Spezialreisebüros/- Anbieter, und Rollstuhl/Behindertenverein. Häufigkeiten sowie erwartete Häufigkeiten sind in Abbildung 18 dargestellt. Die schwarzen Balken zeigen die beobachteten Häufigkeiten und die grauen Balken zeigen die erwarteten Häufigkeiten. 


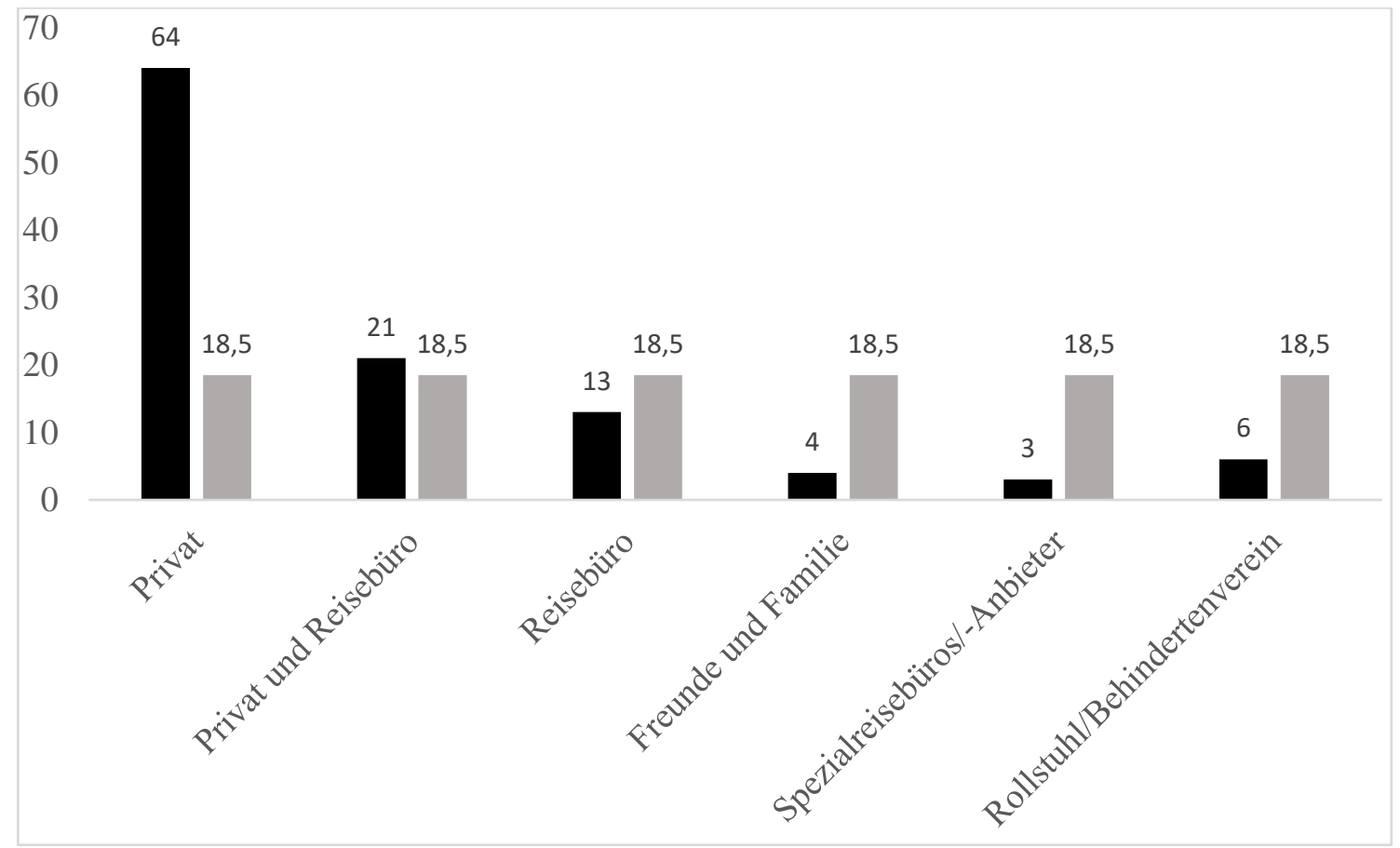

Abbildung 18: Arten, wie der vergangene Urlaub gebucht wurde.

Der Chi-Quadrat Test ergab, dass die unterschiedlichen Buchungsarten unterschiedlich häufig verwendet wurden $(p<.001)$. Abbildung 18 zeigt, dass die Unterschiede zwischen privaten Buchungen und allen anderen Buchungsmethoden größer sind als alle anderen Vergleiche zwischen den Buchungsarten. Die beobachteten Häufigkeiten unterscheiden sich signifikant voneinander und sie zeigen eindeutig höhere Zahlen bei privater Buchung und der mischvariante von Privat und Reisebüro. Daraus kann geschlossen werden, dass die Private Buchung häufiger genutzt wird als sonstige Wege und die Hypothese 4 kann somit bestätigt werden: Menschen mit Körperbehinderung buchen ihren Urlaub häufiger privat als über sonstige Wege.

Chi-Quadrat Test zur Bestimmung von Informationsquellen hinsichtlich der Urlaubsplanung

Mit einem eindimensionalen Chi-Quadrat Test wird analysiert, ob die ProbandInnen unterschiedliche Informationsquellen für ihren letzten Urlaub nutzten. Die vorgegebenen Möglichkeiten waren: 
1) Empfehlungen von Bekannten/Freunden oder anderen Personen mit Mobilitätseinschränkung

2) Spezialreiseveranstalter für Menschen mit Behinderung

3) Homepage/Internet

4) Reiseführer speziell für Menschen mit Behinderung

5) Selbsthilfegruppe/Vereine

6) Kataloge

7) Reisebüro

8) Beherbergungsbetrieb

9) Keine

Fremdenverkehrsbüros waren auch eine Option, die von niemanden genutzt wurde. Häufigkeiten und erwartete Häufigkeiten sind in Abbildung 18 dargestellt. Die Nummerierungen auf der Grafik sind von der Aufzählung übernommen. Die schwarzen Balken zeigen die beobachteten Häufigkeiten und die grauen Balken zeigen die erwarteten Häufigkeiten.

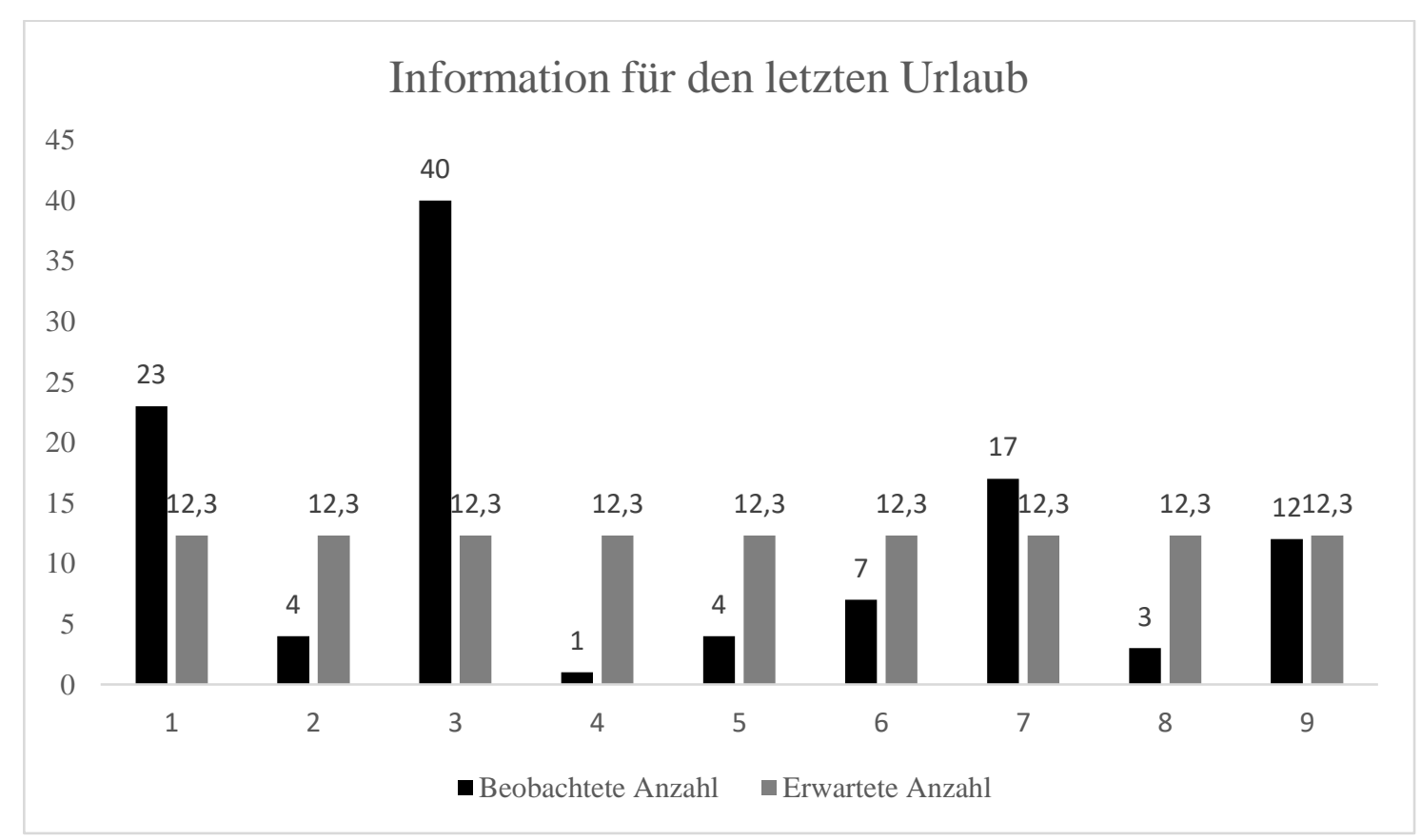

Abbildung 19: Arten der Informationseinholung für eine barrierefreie Reise 
Der Chi-Quadrat Test ergab, dass die Informationsquellen für eine Urlaubsplanung unterschiedlich häufig verwendet wurden $(p<.001)$. Abbildung 19 zeigt, dass die Option Homepage/Internet am häufigsten verwendet wurde. Die beobachteten Häufigkeiten unterscheiden sich signifikant voneinander und sie zeigt eindeutig höhere Zahlen bei der Option Homepage/Internet. Daraus kann geschlossen werden, dass das Internet am häufigsten als wichtige Informationsquelle für die Urlaubsplanung genannt wird und die Hypothese 5 kann somit bestätigt werden: Das Internet wird am häufigsten als wichtige Informationsquelle, für die Planung des letzten barrierefreien Urlaubs genannt.

\section{Chi-Quadrat Test zur Bestimmung der Geschlechterunterschiede bezüglich der Reiseassistenz}

Mit dem Chi-Quadrat-Test wird analysiert, ob mehr Frauen als Männer Reiseassistenz in Anspruch nehmen. Es gab die Möglichkeit weiblich, männlich und keine Angabe zu wählen, da die Möglichkeit besteht, dass Personen an der Studie teilnehmen, die sich nicht den beiden Geschlechterkategorien zugehörig fühlen. Daraus resultiert ein $N$ von 107. In Abbildung 20 wird aufgezeigt, wie viele Männer und Frauen Reiseassistenz in Anspruch nehmen. Das wird jeweils im schwarzen Balken abgebildet. 13 Männer und 13 Frauen haben Reiseassistenz in Anspruch genommen. Die grauen Balken zeigen die Anzahl der Personen an, die keine Assistenz in Anspruch genommen haben. 


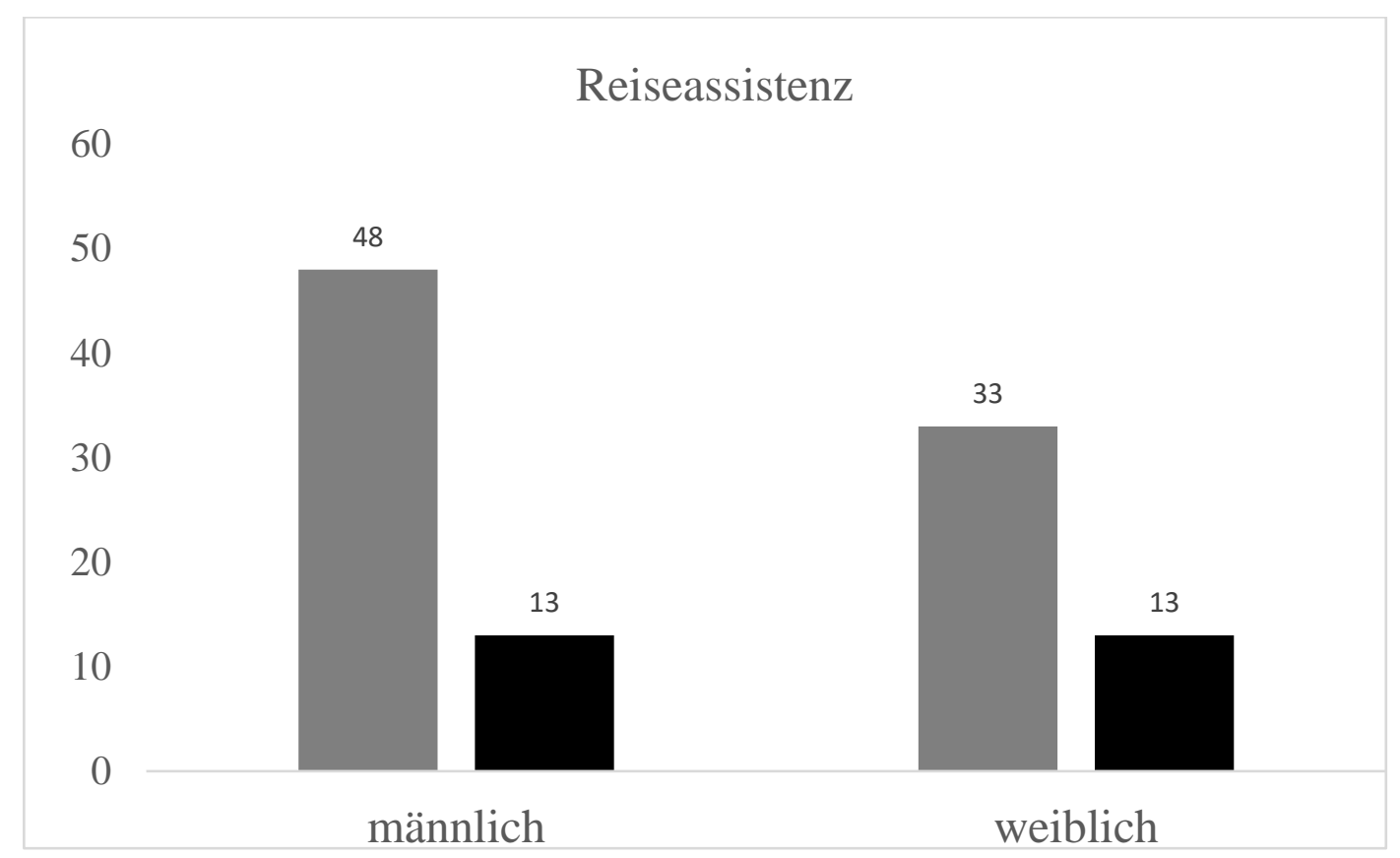

Abbildung 20: Inanspruchnahme von Reiseassistenz.

Der Chi-Quadrat Test ergab, dass keine signifikanten Unterschiede hinsichtlich der Inanspruchnahme von Reiseassistenz zwischen Männer und Frauen bestehen ( $p=.407)$. Daher kann die $\mathrm{H} 1$ nicht bestätigt werden und die H0 trifft zu: Es nehmen nicht mehr Frauen Reiseassistenz in Anspruch als Männer.

\section{Weitere Ergebnisse}

Die weiteren Ergebnisse der Arbeit bieten einen Überblick von konkreten Realisierungsmöglichkeiten einer barrierefreien Reise. Bei der Frage nach der Nutzung von Reiseassistenz, gaben jeweils 9 Personen an, ihre Reise über persönliches Budget, Privat oder einen Verein zu verwirklichen. Die dritte Fragestellung, welche Möglichkeiten zur Realisierung einer Reise werden von Menschen mit Behinderung genutzt, wird mit den nächsten Ergebnissen konkretisiert. Es werden die unterschiedlichen Informationsmaterialien und Buchungsoptionen aus dem Fragebogen angegeben. Die Angaben sind unverändert aus den beantworteten Datensätzen übernommen.

- Runa-Reisen: Reisebüro für barrierefreies Reisen aus Deutschland

- Handicapped-Reisen: Rollstuhlgeeignete Hotels, Pensionen, Ferienbauernhöfe, Ferienwohnungen/-häuser aus Deutschland, Europa allgemein und Übersee. 
- Steiermark für alle: barrierefreie Unterkünfte und Freizeittipps

- ÖBB Homepage

- barrierefrei-reisen: Reiseorganisation von barrierefreien Reisen für SeniorInnen oder Menschen mit körperlicher oder geistiger Beeinträchtigung

- Mare Nostrum: Agentur Für Behindertenreisen Berlin

- wheelmap.org: Online Karte, bei der rollstuhlgerechte Orte weltweit von allen NutzerInnen eingetragen werden können

- roomchooser: Buchungsmöglichkeit von barrierefreien Hotelzimmern

- http://www.rollstuhl-urlaub.de: Auflistung von rollstuhlgerechten und behindertenfreundlichen Urlaubsquartieren in Europa

- grabowski reisen (grabo-reisen): weltweite Reisen, Kreuzfahrten und Safaris für Behinderte Gäste

- Holidays on Wheels: App für barrierefreies Reisen

- http://www.germany.travel/de/barrierefreies-reisen/barrierefreies-reisen/barrierefreies-reisen.html: Urlaub für alle in Deutschland

- reisemeer: Reisebüro für Behindertenreisen in Linz

Die berichteten Ergebnisse bieten einen Überblick über konkrete Anbieter, die für barrierefreie Reisen genutzt werden. Auffällig ist hier, dass viele Links in den Fragebogen eingefügt wurden, also online-Quellen verwendet wurden. Es werden nur wenige Reisebüros angeführt und viele der Anbieter kommen aus Deutschland. 


\section{Interpretation und Diskussion der Ergebnisse mit Implika- tionen für touristische Anbieter und Ausblick für weitere Forschung}

Abschließend werden die Ergebnisse der Hypothesentests und Fragestellungen aufgezeigt, sowie Möglichkeiten für touristische Anbieter geboten, um barrierefreies Reisen hinsichtlich vorkommender Probleme zu verbessern. Auch Beschränkungen der Ergebnisse aufgrund der Repräsentativität der Stichprobe und der gewählten Items sollen dargelegt werden.

Die Methode der Online-Befragung ist kritisch zu betrachten. Auch wenn die Zielgruppe in der Einleitung der Befragung beschrieben wird und nach Unterstützungsmitteln gefragt wird, kann nicht klar verfolgt werden, wer an der Studie teilnimmt und ob einzelne Personen die Befragung mehrmals ausfüllen.

Es wurden zur Beantwortung der ersten beiden Hypothesen die Bereiche Planung, Anund Abreise, Unterkunft und Freizeitangebot aus dem Fragebogen von Flucher at al. (2013) übernommen. Mit diesen Fragen wurde erhoben, welche Probleme am häufigsten in der touristischen Servicekette auftreten und wie sehr diese stören. Es wurden in jedem der Bereiche, die fünf bei Flucher at al. (2013) am häufigsten genannten Probleme herausgenommen, um weniger relevante Probleme bereits vor der Befragung auszuschließen. Da diese fünf Bereiche pro Themenbereich variieren, wurden unterschiedliche Items miteinander verglichen. Hier wäre für zukünftige Forschungen interessant, gleiche Probleme auf den Stationen der touristischen Servicekette zu vergleichen, etwa die Häufigkeit baulicher Barrieren in den Themenbereichen Planung, An- und Abreise sowie Unterkunft und Freizeitangebot.

Hypothese 1: Für Touristische Anbieter ist nicht allein eine barrierefreie Unterkunft relevant. Es wird aufgezeigt, dass die häufigsten Probleme in Verbindung mit dem Freizeitangebot stehen. Informationen über barrierefreie Angebote in der Umgebung können für die Zielgruppe von Vorteil sein. 
Hypothese 2: Es werden Probleme, die mit dem Freizeitangebot im Zusammenhang stehen als am störendsten wahrgenommen. Hier besteht die Möglichkeit barrierefreie Apps wie „Wheelmap“ oder „Holidays on Wheels“ anzuwenden, wenn ein Urlaub geplant wird. Touristische Anbieter haben die Möglichkeit Informationen zur Barrierefreiheit online zur Verfügung zu stellen, wie barrierefreie Toiletten und Maße der Durchgänge und Aufzüge. Damit könnten mehr BesucherInnen das Freizeitangebot nützen, was positive Auswirkungen für die BesucherInnen als auch für den Betrieb haben kann.

Hypothese 3: Die Relevanz der Informationen zeigt hier nicht das Freizeitangebot auf, was anzunehmen wäre, da dort die häufigsten Probleme auftreten, sondern dass Informationen zu An- und Abreise als am wichtigsten einzustufen sind. Hier treten anscheinend weniger Probleme auf, aber im Bereich Informationen sind sie am relevantesten. Das könnte daran liegen, dass dieser Punkt im Rahmen der Planung wichtig ist, denn wenn die An- und Abreise nicht funktioniert, können die ProbandInnen den Urlaubsort gar nicht erreichen. Touristische Anbieter können hier barrierefreie Anfahrtspläne erstellen, die universell designt sind.

Hypothese 4: Es zeigt sich, dass mehr Menschen mit Behinderung ihren Urlaub vorzugsweise privat als über sonstige Wege buchen. Ein Grund dafür könnte sein, dass spezielle Angebote für Menschen mit Behinderung fehlen und die Marktlücke, wie sie von Wilken (2016) beschrieben wird, noch zu schließen ist. Eine weiterführende Forschungsmöglichkeit könnte hier ein Vergleich zwischen Menschen mit und ohne Behinderung sein, da durch die Technisierung viele private Buchungsmöglichkeiten via Internet einfach von zu Hause zu tätigen sind.

Hypothese 5: Das Internet wird am häufigsten als wichtigste Informationsquelle für die Planung des letzten barrierefreien Urlaubs genannt, wie bereits bei der Studie von Laburda \& Smikac (2009). Gründe hierfür können wieder das fehlende Angebot an Reisebüros sein, wie es Wilken (2016) beschreibt, oder dass durch die einfache Informationsbeschaffung auf das Internet zurückgegriffen wird. Es gibt hierfür bereits ein breites Angebot, wie in den Best-Practice-Beispielen beschrieben wird. 
Wenn ein barrierefreier Zugang ohne genauere Angaben auf der Internetseite versprochen wird, kann es trotzdem auf der Reise zu Problemen kommen, da Ansprüche auf Barrierefreiheit variieren, wie es Kastl (2017) beschreibt. Hier können touristische Anbieter mit genauen Maßangaben der Unterkunft behilflich sein.

Hypothese 6: Es nehmen laut Ergebnissen der Studie nicht mehr Frauen Reiseassistenz in Anspruch als Männer. Auffällig ist, dass generell von wenigen TeilnehmerInnen der Studie (26 Personen) Reiseassistenz in Anspruch genommen wird. Hier wäre interessant, wie sehr Familienangehörige die Pflege übernehmen, oder ob viele Menschen mit einer Behinderung des Bewegungsapparates nicht auf Assistenz angewiesen sind. Touristische Anbieter können hier informieren, ob am Urlaubsort direkt Assistenz in Anspruch genommen werden kann.

Bei den weiteren Ergebnissen wird der Planungsbereich näher betrachtet. Es wird nach konkreten Informationsseiten gefragt. Jeweils 9 Personen verwirklichen sich ihre Reise über persönliches Budget, privat oder einen Verein. Bei der Fragestellung, welches Informationsmaterial oder welche Homepages über barrierefreien Tourismus gekannt und genutzt werden, war zu sehen, dass kaum ein touristischer Anbieter doppelt genannt wurde, es sei denn es handelt sich um Betriebe mit einer Monopolstellung, wie die ÖBB. Barrierefreie Reisebüros werden eher aus Deutschland genutzt, wie beispielsweise Runareisen. Grund dafür könnte sein, dass in Österreich zu wenige Anbieter vorhanden sind oder deren Internetpräsenz nicht gut genug ausgebaut ist.

Während der Arbeit wurden Kontakte zwischen "Holidays on Wheels“ und ,atempo“ hergestellt und es wurden deren Daten zu einer großen Plattform zusammengelegt, welche nun über die App von „Holidays on Wheels“ aufgerufen werden kann.

Die Ergebnisse der Arbeit können auch allgemein für Menschen mit Mobilitätseinschränkung interessant sein, da auch andere Personengruppen, wie beispielsweise SeniorInnen, stark übergewichtige Personen oder auch Eltern mit Kinderwagen von einer barrierefreien Umgebung profitieren. 


\section{Literatur}

ADAC (2003). Barrierefreier Tourismus für Alle. Eine Planungshilfe für Tourismus-Praktiker zur erfolgreichen Entwicklung barrierefreier Angebote. München: ADAC.

Barnes, C. (2011). Understanding Disability and The Importance Of Design For All. Journal of Accessibility and Design for all. 1(1) 55-80.

Bayrisches Staatsministerium für Familie, Arbeit und Soziales (2017). Woran erkennt man „Produkte für alle“?. Verfügbar unter https://www.barrierefrei.bayern.de/beispiele/universelles-design/index.php [20.08.2018].

Bereit für Barrierefreiheit (2017). Bereit für Barrierefreiheit. Verfügbar unter http://www.bereit-fuer-barrierefreiheit.eu/de/Betriebe/ [28.09.2018].

Bereit für Barrierefreiheit 2 (2017). Bereit für Barrierefreiheit Betriebe. Verfügbar unter http://www.bereit-fuer-barrierefreiheit.eu/de/Betriebe/?xt=CEDOSCheckResults\&xtMethod=showDataset\&cat_id=959\&tobj_id=2848\&show_details=1\&show_catalogue=1\#catalogue [27.09.2018].

BMASK (Bundesministerium für Arbeit, Soziales, Gesundheit und Konsumentenschutz) (2013). Statistiken. Verfügbar unter https://www.sozialministerium.at/cms/site/attachments/5/1/5/CH3434/CMS1450699435356/statistik_-_menschen_mit_behinderung_20131.pdf [29.7.2018]. 
DIMDI (Deutsches Institut für Medizinische Dokumentation und Information) (2005). ICF - Internationale Klassifikation der Funktionsfähigkeit, Behinderung und Gesundheit. Verfügbar unter https://www.dimdi.de/dynamic/de/klassifikationen/icf/ [23.7.2018].

Deutsche Bahn (2018). Bahnbilder. Verfügbar unter http://www.bahnbilder.de/bild/deutschland personenwagen ic-doppelstock-zwischenwagenic-2/915701/an-einem-bahnsteig-mit-einer-hoehe.html. [15.09.2018].

Dederich, M. (2016). Selbstbestimmung. In G. Antor, I. Beck, U. Bleidick \& M. Dederich (Hrsg.), Handlexikon der Behindertenpädagogik. Schlüsselbegriffe und Theorie und Praxis (S. 169-171). Stuttgart: Kohlhammer Verlag.

ENAT (European Network for Accessible Tourism) (2007). Rights of Tourists with Disabilities in the European Union Framework. Verfügbar unter http://www.accessibletourism.org/resources/enat_study_1_rights_final_en.pdf [20.9.2016].

EUCAN (European Concept for Accessibility Network) (2003). European Concept für Accessibility. Verfügbar unter www.eca.lu [11.11.2016].

Eurostat (2016). Pressemitteilung. Verfügbar unter http://ec.europa.eu/eurostat/documents/2995521/7755884/3-02122016-BP-DE.pdf/d17a2b1a-cf4047a1-97cb-5b9bd5806e96 [29.07.2018].

Fissler, M. (2015). Universal Design im Kontext von Inklusion und Teilhabe. Internationale Eindrücke und Perspektiven. Recht und Praxis. (2) 45-52. 
Flucher, B., Parigger, L. \& Zirngast, A. (2013). Barrierefreier Urlaub in Österreich. Eine quantitative Analyse der auftretenden Probleme von Rollstuhlfahrerinnen entlang der touristischen Servicekette. (Unveröffentl. Seminararbeit). Karl-Franzens-Universität, Graz.

Fojan, A. (2018). Holidays on Wheels. Verfügbar unter https://www.startnext.com/holidaysonwheels. [12.6.2018].

Franz, A. (2002). Selbstbestimmt Leben mit Persönlicher Assistenz. Eine alternative Lebensform behinderter Frauen. Dortmund: Mobile.

Gruber, H., \& Ledl V. (2004). Allgemeine Sonderpädagogik. Ein Studien- und Abeitsbuch. St. Stefan: Jugend und Volk Verlag.

Hackl, J. (2014). Persönliche Assistenz und Lebensqualität bei behinderten Menschen. Hamburg: Diplomica Verlag.

Höglinger, S. (2010). Barrierefreier Tourismus und die Rolle des Reisemittlers. Wien: Peter Lang Verlag.

Hülshoff, T. (2011). Basiswissen Medizin für die Soziale Arbeit. München: Ernst Reinhardt Verlag.

Israeli, A. (2002). A Preliminary Investigation of the Importance of Site Accessibility Factors for Disabled Tourist. Journal of Travel Research (41) 101-104.

Kastl, J. M. (2017). Einführung in die Soziologie der Behinderung. Springer: Wiesbaden. 
Land Steiermark (2018). Persönliches Budget. Verfügbar unter http://www.soziales.steiermark.at/cms/beitrag/11843358/439/ [12.10.2017].

Laburda, A., \& Smikac H. (2009). Reisepläne und Aktivitäten für einen Urlaub ohne Barrieren. Verfügbar unter http://ibft.at/upload/Ergebnisbericht_Umfrage-2008-09.html [11.11.2016].

Leidner, M. (2015). Verschiedenheit, besondere Bedürfnisse und Inklusion. Grundlagen der Heilpädagogik. Hohengehren: Schneider Verlag.

Leitner, B. (2008). Menschen mit Beeinträchtigung. Ergebnisse der MikrozensusZusatzfragen im 4. Quartal 2007. Statistische Nachrichten (12) 1132-1141.

Link, C. (2016). Behindert ist man nicht, Behindert wird man. Verfügbar unter http://www.zeit.de/video/2016-09/5111872335001/z2x-behindert-ist-mannicht-behindert-wird-man [11.10.2017].

Leyendecker, C. (2005). Motorische Behinderungen. Grundlagen, Zusammenhänge und Förderungsmöglichkeiten. Stuttgart: Kohlhammer Verlag.

$\begin{array}{llll}\text { Oorlogsmuseum } & \text { (2017). } & \text { Verfügbar }\end{array}$ https://venlo.nieuws.n1/nieuws/34812/oorlogsmuseum-overloon-gratis-bezoeken/ [13.06.2018].

Österreichisches Normungsinstitut (2005). Barrierefreies Bauen - Planungsgrundlagen. Verfügbar unter https://www.bauberufe.eu/images/doks/_Oenormb1600.pdf [10.05.2018]. 
Pfeiffer E. (2010). Vortrag im Rahmen der Fachtagung „,Tourismus für Alle-Design for All. Herausforderung für Technologie- und Tourismusentwicklung “ an der TU München. Verfügbar unter https://www.behindertenbeauftragteoal.de/fileadmin/PDF/Barrierefreier_Tourismus/Tourismusfachtagung_2010/Tourismusfachtagung_Muenchen_Herr_Pfeiffer.pdf [19.4.2019].

Puschke, M. (2005). Die internationale Klassifikation von Behinderung der Weltgesundheitsorganisation. Weiber ZEIT, Zeitung des Projektes „Politische Interessensvertretung behinderter Frauen“ des Weibernetzes, April 2005 (7). 45.

Rannegger, R. (2006). Barrierefreier Tourismus in Österreich. Analyse des Reiseverhaltens und den dabei auftretenden Problemen entlang der touristischen Servicekettim Rollstuhl aus Österreich. (Unveröffentlichte Diplomarbeit). IMC Fachhochschule Krems.

Rasch, B., Friese, M., Hofmann, W. \& Naumann E. (2010). Quantitative Methoden. Band 2 (3. Auflage). Heidelberg: Springer.

Rock, K. (2001). Sonderpädagogische Professionalität unter der Leitidee der Selbstbestimmung. Bad Heilbronn: Julius Klinkhardt Verlag.

Sozialministerium (2016). Broschürenservice. https://broschuerenservice.sozialministerium.at/Home/Download?publicationId=19_[15.12.2017]. 
Theunissen, G. (2013). Empowerment und Inklusion behinderter Menschen. Eine Einführung in Heilpädagogik und Soziale Arbeit. 3. Auflage, Lambertus Verlag: Freiburg.

Theunissen, G. (2016). Empowerment. In: M. Dederich, I. Beck, G. Antor \& U. Bleidick (Hrsg.), Handlexikon der Behindertenpädagogik (S. 113-117). Schlüsselbegriffe aus Theorie und Praxis. 3. Auflage. Stuttgart: Kohlhammer.

Weber, E. (2016). Assistenz. In: G. Biewer, I. Hedderich, J. Hollenweger, \& R. Markowetz (Hrsg.), Handbuch Inklusion und Sonderpädagogik (S. 158). Regensburg: Verlag Friedrich Pustet.

Wilken U. (2015) Freizeit für alle - barrierefrei. In: R. Freericks, D. Brinkmann (Hrsg.), Handbuch Freizeitsoziologie (S. 467-488). Wiesbaden: Springer Verlag.

Wilken, U. (2016). Herausforderungen bei der Gestaltung und Vermarktung eines barrierefreien Tourismus. Ein zukunftsoffenes Resümee nach 40 Jahren. Zeitschrift für Tourismuswirtschaft. 8(1), 145-156.

Wrussnig, K. (2010). BARRIERE(FREIHEIT) IM TOURISMUS?. (Masterarbeit). Karl-Franzens-Universität, Graz. 


\section{Abbildungsverzeichnis}

Abbildung 1: Wechselwirkungen zwischen den Komponenten der ICF (DIMDI 2005, 23)....... 7

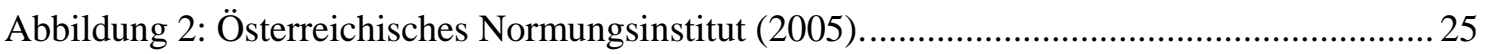

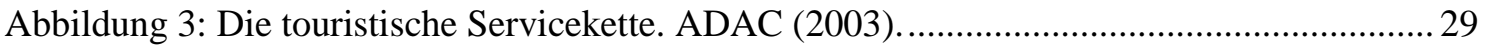

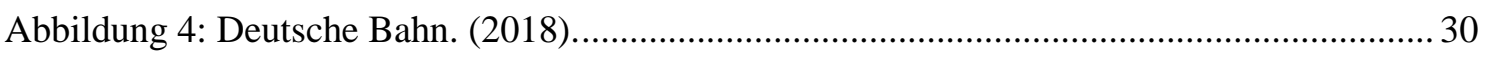

Abbildung 5: Bayrisches Staatsministerium für Familie, Arbeit und Soziales (2017). ............... 31

Abbildung 6: Bayrisches Staatsministerium für Familie, Arbeit und Soziales (2017). .............. 31

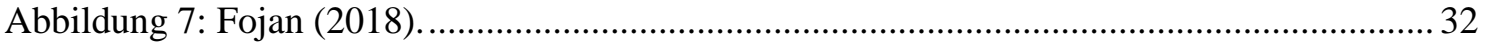

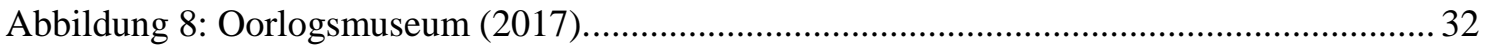

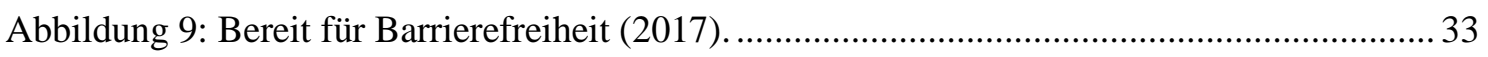

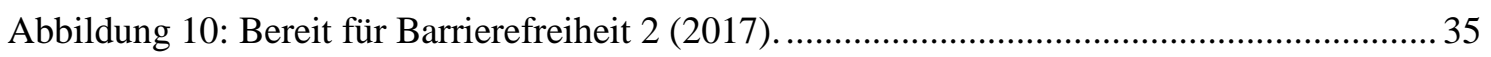

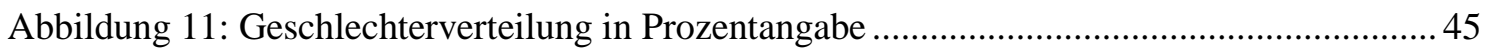

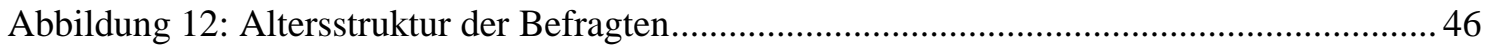

Abbildung 13: Herkunft der TeilnehmerInnen......................................................................... 47

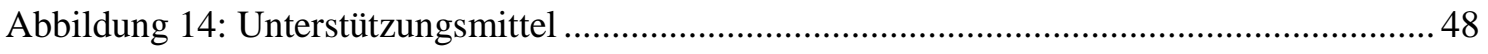

Abbildung 15: Problemaufkommen in den Bereichen: Planung, An- und Abreise, Unterkunft und

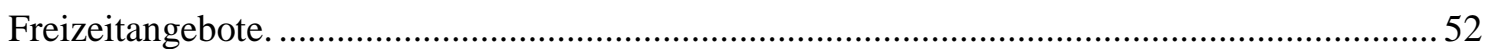

Abbildung 16: Problembewertung der Felder Planung, An- und Abreise, Unterkunft und

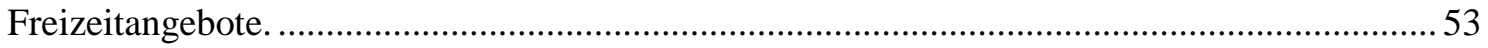

Abbildung 17: Relevanz der Informationen bezüglich Planung, An- und Abreise, Unterkunft und

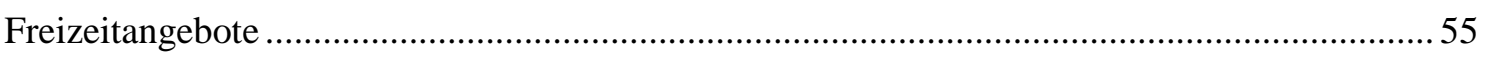

Abbildung 18: Arten, wie der vergangene Urlaub gebucht wurde.............................................56

Abbildung 19: Arten der Informationseinholung für eine barrierefreie Reise ........................... 57

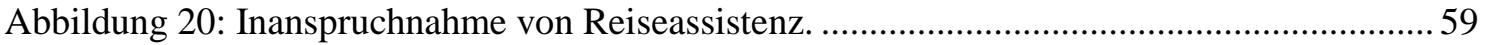




\section{Anhang}

\section{Fragebogen}

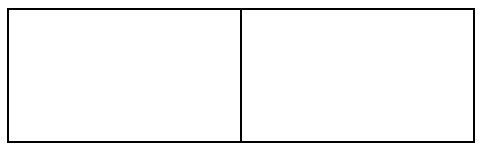

Karl-Franzens-Universität Graz

Institut für Erziehungs- und Bildungswissenschaft

Arbeitsbereich Integrationspädagogik und Heilpädagogische Psychologie

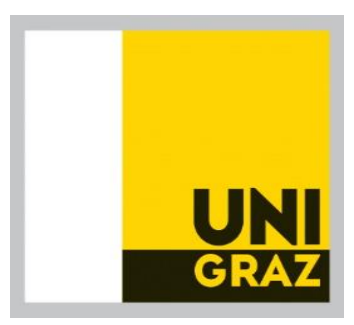

\section{Fragebogen}

\section{Barrierefreier Urlaub für Menschen mit Mobilitätseinschränkung}

\section{ab 18 Jahre}

Liebe Teilnehmerin/Lieber Teilnehmer

Im Rahmen meiner Masterarbeit zum Thema Barrierefreien Tourismus am Institut für Erziehungs- und Bildungswissenschaft der Karl-Franzens-Universität Graz untersuche ich mögliche Barrieren und Realisierungsmöglichkeiten für Menschen mit einer Mobilitätseinschränkung, die eine Reise Planen. Die Masterarbeit wird unter der Betreuung von Prof. Dr. Peter Rossmann verfasst.

Alle ihre angegebenen Daten und Antworten werden vertraulich und anonym behandelt und werden niemanden mitgeteilt. Bitte beantworten Sie alle Fragen vollständig, gewissenhaft und wahrheitsgemäß. Das Ausfüllen des gesamten Fragebogens wird etwa 15 Minuten ihrer Zeit in Anspruch nehmen.

Ich möchte mich herzlich bei Ihnen dafür bedanken, dass Sie sich für den Fragebogen Zeit nehmen und Auskunft über Ihr Reiseverhalten geben. Erst durch Ihre Angaben wird die Untersuchung des Themas möglich.

Bei Fragen oder Anmerkungen zum Fragebogen bzw. bei Interesse über die Ergebnisse der Studie, kontaktieren Sie mich bitte unter der E-Mail-Adresse: ka.platzer@edu.uni-graz.at

Mit freundlichen Grüßen,

Karin Platzer, BA 
Im folgenden Fragebogen finden Sie eine Reihe von Fragen zum Thema Barrierefreien Tourismus, beginnend mit dem Thema Reiseassistenz.

1. Haben Sie für ihren letzten Urlaub Reiseassistenz in Anspruch genommen?

Nein

2. Wenn ja, über

Verein

Privat

Persönliches Budget

Sonstiges

3. Wie sind Sie zu den nötigen Informationen gekommen, um Assistenzleistungen zu organisieren?

Die folgenden Fragen beinhalten die Themen Buchung und Reiseplanung.

4. Wie haben Sie Ihren letzten Urlaub gebucht? Bitte nennen Sie nur die wichtigste Option.

Privat

Privat und Reisebüro

Reisebüro

Freunde und Familie

Spezialreisebüros/-Anbieter

Rollstuhl/-Behindertenverein

5. Woher hatten Sie die Informationen für die Planung Ihres letzten Urlaubes? Bitte nennen Sie nur die wichtigste Option.

Empfehlungen von Bekannten/Freunden Spezialreiseveranstalter für Menschen mit Behinderung

oder anderen Personen mit

Mobilitätseinschränkung

Homepage/Internet

Reiseführer speziell für Menschen mit Behinderung

Selbsthilfegruppe/Vereine

Fremdenverkehrsbüro

Kataloge Reisebüro

Beherbergungsbetrieb Keine

6. Nennen Sie nun weitere wichtige Planungsoptionen für die Urlaubsplanung Ihres letzten Urlaubs.

Kreuzen Sie nun bitte nur weitere Planungsarten an, die für Sie wichtig sind. 
○ Empfehlungen von

Bekannten/Freunden oder anderen

Personen

mit
○ Spezialreiseveranstalter für Menschen mit Behinderung

Mobilitätseinschränkung

$\begin{array}{ll}\text { Homepage/Internet } & \text { Reiseführer speziell für Menschen mit } \\ \text { Behinderung } & \\ \text { Selbsthilfegruppe/Vereine } & \text { Fremdenverkehrsbüro } \\ \text { Kataloge } & \text { Reisebüro } \\ \text { Beherbergungsbetrieb } & \text { Keine }\end{array}$

7. Welche Onlineseiten verwenden Sie für Ihre Urlaubsplanung?

$$
\text { ○ }
$$

○ keine 


\section{8 bis 12: Themenbereich Planung}

Mit welchen Problemen waren Sie vor Ihrem letzten Urlaub konfrontiert?

Bitte geben Sie an, mit welchen Problemen Sie konfrontiert waren und wie störend Sie diese empfunden haben. Nur im Fall einer Ja-Antwort geben Sie bitte an wie sehr es Sie gestört hat.

\begin{tabular}{|c|c|c|c|c|}
\hline & & Das & Problem & $\begin{array}{l}\text { Gewichtung } \\
\text { Es hat mich... }\end{array}$ \\
\hline 8. & $\begin{array}{l}\text { Das Reisebüro, in dem ich gebucht habe, } \\
\text { war für Menschen mit } \\
\text { Mobilitätseinschränkung nicht bzw. nur } \\
\text { schwer zugänglich? }\end{array}$ & O nein & $\mathrm{O} \mathrm{ja}=>$ & $\begin{array}{l}\text { O nicht gestört } \mathrm{O} \text { wenig gestört } \\
\text { O eher gestört O sehr gestört }\end{array}$ \\
\hline 9. & $\begin{array}{l}\text { Fehlende Kompetenz und Sensibilität } \\
\text { der MitarbeiterInnen im Reisebüro. }\end{array}$ & O nein & $\mathrm{O} j \mathrm{ja}=>$ & $\begin{array}{l}\text { O nicht gestört } \mathrm{O} \text { wenig gestört } \\
\text { O eher gestört } \mathrm{O} \text { sehr gestört }\end{array}$ \\
\hline 10. & $\begin{array}{l}\text { Fehlende, zu ungenaue oder falsche } \\
\text { Informationen vom Reisebüro über die } \\
\text { Zugänglichkeit/Barrierefreiheit am } \\
\text { Urlaubsort. }\end{array}$ & O nein & $\mathrm{O} \mathrm{ja} \Rightarrow$ & $\begin{array}{l}\text { O nicht gestört } \mathrm{O} \text { wenig gestört } \\
\text { O eher gestört O sehr gestört }\end{array}$ \\
\hline 11. & $\begin{array}{l}\text { Homepages/Broschüren der } \\
\text { Urlaubsanbieter und } \\
\text { Transportunternehmen gehen nicht auf } \\
\text { die Bedürfnisse von Menschen mit } \\
\text { Mobilitätseinschränkung ein. }\end{array}$ & O nein & $\mathrm{O} j \mathrm{ja} \Rightarrow$ & $\begin{array}{l}\text { O nicht gestört } \mathrm{O} \text { wenig gestört } \\
\text { O eher gestört } \mathrm{O} \text { sehr gestört }\end{array}$ \\
\hline 12. & $\begin{array}{l}\text { Zu genaue oder falsche Informationen } \\
\text { (z.B.: via Telefon, E-Mail, Internet) vom } \\
\text { Urlaubsanbieter über die } \\
\text { Zugänglichkeit/Barrierefreiheit am } \\
\text { Urlaubsort. }\end{array}$ & O nein & $\mathrm{O} \mathrm{ja} \Rightarrow>$ & $\begin{array}{l}\text { O nicht gestört } \mathrm{O} \text { wenig gestört } \\
\text { O eher gestört O sehr gestört }\end{array}$ \\
\hline
\end{tabular}


13 bis 17: Themenbereich An-Abreise

Mit welchen Problemen bezüglich der An- und Abreise waren Sie während Ihres letzten Urlaubs konfrontiert?

Bitte geben Sie an, mit welchen Problemen Sie konfrontiert waren und wie störend Sie diese empfunden haben. Nur im Fall einer Ja-Antwort geben Sie bitte an wie sehr es Sie gestört hat.

\begin{tabular}{|c|c|c|c|c|}
\hline & & Das & Problem & $\begin{array}{l}\text { newichtung } \\
\text { Es hat mich... }\end{array}$ \\
\hline 13. & $\begin{array}{l}\text { Ein- und Ausstieg beim (Reise-) } \\
\text { Bus/Zug für Menschen mit } \\
\text { Mobilitätseinschränkung nicht bzw. } \\
\text { nur schwer möglich. }\end{array}$ & O nein & & $\begin{array}{l}\text { O nicht gestört } \mathrm{O} \text { wenig gestört } \\
\text { O eher gestört } \mathrm{O} \text { sehr gestört }\end{array}$ \\
\hline 14. & $\begin{array}{l}\text { Kein Platz/keine Stellmöglichkeit } \\
\text { im Zug/Bus für Menschenmit } \\
\text { Mobilitätseinschränkung, } \\
\text { während der Fahrt im Rollstuhl } \\
\text { sitzen bleiben wollen. }\end{array}$ & O nein & $\mathrm{O}$ ja $\Rightarrow$ & $\begin{array}{l}\text { O nicht gestört } \mathrm{O} \text { wenig gestört } \\
\text { O eher gestört O sehr gestört }\end{array}$ \\
\hline 15. & $\begin{array}{l}\text { Sanitäre Einrichtungen im Zug/Bus } \\
\text { nicht bzw. nur schwer benutzbar. }\end{array}$ & O nein & $\mathrm{O} \mathrm{ja}=>$ & $\begin{array}{l}\text { O nicht gestört } \mathrm{O} \text { wenig gestört } \\
\text { O eher gestört } \mathrm{O} \text { sehr gestört }\end{array}$ \\
\hline 16. & $\begin{array}{lll}\text { Fahrkartenautomat } & \text { oder } \\
\text { Fahrkartenschalter nicht } & \text { oder } \\
\text { schwer erreichbar. } & & \end{array}$ & O nein & $\mathrm{O} \mathrm{ja}=>$ & $\begin{array}{l}\text { O nicht gestört } \mathrm{O} \text { wenig gestört } \\
\text { O eher gestört O sehr gestört }\end{array}$ \\
\hline 17. & $\begin{array}{l}\text { Bauliche Barrieren innerhalb des } \\
\text { Bahnhofbereiches. }\end{array}$ & O nein & $\mathrm{O} \mathrm{ja}=>$ & $\begin{array}{l}\text { O nicht gestört } \mathrm{O} \text { wenig gestört } \\
\text { O eher gestört O sehr gestört }\end{array}$ \\
\hline
\end{tabular}




\section{8 bis 22: Themenbereich Unterkunft}

Mit welchen Problemen bezüglich der Unterkunft, waren Sie während Ihres letzten Urlaubs konfrontiert?

Bitte geben Sie an, mit welchen Problemen Sie konfrontiert waren und wie störend Sie diese empfunden haben. Nur im Fall einer Ja-Antwort geben Sie bitte an wie sehr es Sie gestört hat.

\begin{tabular}{|c|c|c|c|}
\hline & & $\begin{array}{l}\text { Das Problem hatt } \\
\text { ich }\end{array}$ & $\begin{array}{l}\text { Gewichtung } \\
\text { Es hat mich... }\end{array}$ \\
\hline 18. & $\begin{array}{l}\text { Bauliche Barrieren zur } \\
\text { bzw. innerhalb der } \\
\text { Unterkunft. }\end{array}$ & $\mathrm{O}$ nein $\mathrm{O} \mathrm{ja} \Rightarrow$ & $\begin{array}{l}\mathrm{O} \text { nicht gestört } \mathrm{O} \text { wenig gestört } \\
\mathrm{O} \text { eher gestört } \mathrm{O} \text { sehr gestört }\end{array}$ \\
\hline 19. & $\begin{array}{l}\text { Schwergängige Türen } \\
\text { oder Drehtüren. }\end{array}$ & $\mathrm{O}$ nein $\mathrm{O} \mathrm{ja}=>$ & $\begin{array}{l}\mathrm{O} \text { nicht gestört } \mathrm{O} \text { wenig gestört } \\
\text { O eher gestört } \mathrm{O} \text { sehr gestört }\end{array}$ \\
\hline 20. & $\begin{array}{l}\text { Eingeschränkte } \\
\text { Bewegungsfläche in den } \\
\text { Zimmern. }\end{array}$ & O nein $\mathrm{O} j \mathrm{ja} \Rightarrow$ & $\begin{array}{l}\mathrm{O} \text { nicht gestört } \mathrm{O} \text { wenig gestört } \\
\mathrm{O} \text { eher gestört } \mathrm{O} \text { sehr gestört }\end{array}$ \\
\hline 21. & $\begin{array}{l}\text { Möblierung im Zimmer } \\
\text { nicht rollstuhlgerecht } \\
\text { positioniert bzw. nicht } \\
\text { unterfahrbar. }\end{array}$ & $O$ nein $O \mathrm{ja} \Rightarrow$ & $\begin{array}{l}\mathrm{O} \text { nicht gestört } \mathrm{O} \text { wenig gestört } \\
\mathrm{O} \text { eher gestört } \mathrm{O} \text { sehr gestört }\end{array}$ \\
\hline 22. & $\begin{array}{l}\text { Badezimmer nicht } \\
\text { rollstuhlgerecht } \\
\text { eingerichtet. }\end{array}$ & $\mathrm{O}$ nein $\mathrm{O} \mathrm{ja}=>$ & $\begin{array}{l}\mathrm{O} \text { nicht gestört } \mathrm{O} \text { wenig gestört } \\
\mathrm{O} \text { eher gestört } \mathrm{O} \text { sehr gestört }\end{array}$ \\
\hline
\end{tabular}




\section{3-27: Themenbereich Freizeitangebot}

Mit welchen Problemen bezüglich des Freizeitangebotes waren Sie während Ihres letzten Urlaubs konfrontiert?

Bitte geben Sie an, mit welchen Problemen Sie konfrontiert waren und wie störend Sie diese empfunden haben. Nur im Fall einer Ja-Antwort geben Sie bitte an wie sehr es Sie gestört hat.

\begin{tabular}{|c|c|c|c|}
\hline & & $\begin{array}{l}\text { Das Problem hatt } \\
\text { ich }\end{array}$ & $\begin{array}{l}\text { Gewichtung } \\
\text { Es hat mich... }\end{array}$ \\
\hline 23. & $\begin{array}{l}\text { Sanitäre Einrichtungen am Ausflugsziel } \\
\text { nicht bzw. nur erschwert benutzbar. }\end{array}$ & $\mathrm{O}$ nein $\mathrm{O} \mathrm{ja}=>$ & $\begin{array}{l}\text { O nicht gestört } \mathrm{O} \text { wenig gestört } \\
\mathrm{O} \text { eher gestört } \mathrm{O} \text { sehr gestört }\end{array}$ \\
\hline 24. & $\begin{array}{l}\text { Teilnahmemöglichkeit } \\
\text { Freizeitangeboten nicht bzw. nur } \\
\text { erschwert möglich. }\end{array}$ & $O$ nein $O \mathrm{ja}=$ & $\begin{array}{l}\text { O nicht gestört } \mathrm{O} \text { wenig gestört } \\
\text { O eher gestört } \mathrm{O} \text { sehr gestört }\end{array}$ \\
\hline 25 . & $\begin{array}{l}\text { Kaum Serviceangebote für Menschen } \\
\text { mit Mobilitätseinschränkung. }\end{array}$ & $O$ nein $O \mathrm{ja}=>$ & $\begin{array}{l}\mathrm{O} \text { nicht gestört } \mathrm{O} \text { wenig gestört } \\
\mathrm{O} \text { eher gestört } \mathrm{O} \text { sehr gestört }\end{array}$ \\
\hline 26. & $\begin{array}{l}\text { Fehlende Informationen über } \\
\text { barrierefreie Angebote vor Ort. }\end{array}$ & $\mathrm{O}$ nein $\mathrm{O} \mathrm{ja} \Rightarrow$ & $\begin{array}{l}\text { O nicht gestört } \mathrm{O} \text { wenig gestört } \\
\mathrm{O} \text { eher gestört } \mathrm{O} \text { sehr gestört }\end{array}$ \\
\hline 27. & $\begin{array}{l}\text { MitarbeiterInnen der touristischen } \\
\text { Betriebe kennen Bedürfnisse von } \\
\text { Menschen mit Mobilitätseinschränkung } \\
\text { nicht. }\end{array}$ & $\mathrm{O}$ nein $\mathrm{O} \mathrm{ja}=>$ & $\begin{array}{l}\text { O nicht gestört } \mathrm{O} \text { wenig gestört } \\
\text { O eher gestört } \mathrm{O} \text { sehr gestört }\end{array}$ \\
\hline
\end{tabular}


28-36: Fragen zur Information über Planung/Unterkunft/An- Abreise und Freizeitangebot

28. Wie wichtig sind Ihnen Informationen im Bereich Planung von barrierefreien Reisen?
- Sehr wichtig
- Eher wichtig
$\circ \quad$ Wenig wichtig
○ Nicht wichtig

29. Kennen Sie Informationsmaterial/Homepages über barrierefreie Urlaubsplanung?

30. Wenn Sie über Reisebüros Ihren Urlaub Planen, über welche?

31. Wie wichtig sind Ihnen Informationen über Barrierefreiheit der Unterkunft?
- Sehr wichtig
- Eher wichtig
$\circ \quad$ Wenig wichtig
○ Nicht wichtig

32 Kennen Sie Informationsmaterial/Homepages über Barrierefreiheit bei Unterkünften?

33. Wie wichtig sind Ihnen Informationen über barrierefreie Anreise?
○ Sehr wichtig
○ Eher wichtig
$\circ \quad$ Wenig wichtig
○ Nicht wichtig

34. Kennen Sie Informationsmaterial/Homepages über barrierefreie Transportmittel (Bahn, Bus...)?

35. Wie wichtig sind Ihnen Informationen über barrierefreie Ausflugziele?
○ Sehr wichtig
- Eher wichtig
$\circ \quad$ Wenig wichtig
$\circ \quad$ Nicht wichtig

36. Kennen Sie Informationsmaterial/Homepages über barrierefreie Ausflugziele? 


\section{Allgemeine Informationen}

37. Geschlecht:

○ weiblich

38. Alter:

39. In welchem Bundesland wohnen Sie:

40. Welche Unterstützungsmittel benötigen Sie:
○ Manueller Rollstuhl
○ Elektrischer Rollstuhl
○ Rollator
○ Unterarmstützen/ Krücken
$\circ$ sonstige
○ keine

○ männlich

- ohne Angabe

\section{Vielen Dank!}




\section{Tabellen der statistischen Datenanalyse}

\section{Tabellen zur statistischen Analyse von Hypothese 1: Problemaufkommen}

\section{Mauchly-Test auf Sphärizität ${ }^{\mathrm{a}}$}

Maß: MEASURE_1

\begin{tabular}{|c|c|c|c|c|c|c|c|}
\hline \multirow[b]{2}{*}{$\begin{array}{l}\text { Innersubjektef- } \\
\text { fekt }\end{array}$} & \multirow[b]{2}{*}{ Mauchly-W } & \multirow{2}{*}{$\begin{array}{l}\text { Näherungs- } \\
\text { weise Chi- } \\
\text { Quadrat }\end{array}$} & \multirow[b]{2}{*}{$\mathrm{df}$} & \multirow[b]{2}{*}{ Sig. } & \multicolumn{3}{|l|}{ Epsilon $^{b}$} \\
\hline & & & & & $\begin{array}{l}\text { Greenhouse- } \\
\text { Geisser }\end{array}$ & $\begin{array}{l}\text { Huynh- } \\
\text { Feldt (HF) }\end{array}$ & $\begin{array}{l}\text { Unter- } \\
\text { grenze }\end{array}$ \\
\hline $\begin{array}{l}\text { Problemaufkom- } \\
\text { men }\end{array}$ & ,860 & 16,413 & 5 & ,006 & ,922 & ,948 & ,333 \\
\hline
\end{tabular}

Testet die Nullhypothese, dass die Fehlerkovarianzmatrix der orthonormalisierten transformierten abhängigen Variablen proportional zu einer Identitätsmatrix ist.

a. Design: Konstanter Term Innersubjektdesign: Problemaufkommen

b. Kann für die Anpassung der Freiheitsgrade für die gemittelten Tests auf Signifikanz verwendet werden. Korrigierte Tests werden in der Tabelle 'Tests der Innersubjekteffekte' angezeigt.

\section{Paarweise Vergleiche}

Maß: MEASURE_1

\begin{tabular}{|c|c|c|c|c|c|c|}
\hline \multirow{2}{*}{$\begin{array}{l}\text { (I) Prob- } \\
\text { lemauf- } \\
\text { kommen }\end{array}$} & \multirow{2}{*}{$\begin{array}{l}\text { (J) Problemaufkom- } \\
\text { men }\end{array}$} & \multirow{2}{*}{$\begin{array}{l}\text { Mittelwert- } \\
\text { differenz (I- } \\
\text { J) }\end{array}$} & \multirow[b]{2}{*}{$\begin{array}{l}\text { Standardfeh- } \\
\text { ler }\end{array}$} & \multirow[b]{2}{*}{ Sig. ${ }^{\text {b }}$} & \multicolumn{2}{|c|}{$\begin{array}{l}95 \% \text { Konfidenzintervall für } \\
\text { Differenz }\end{array}$} \\
\hline & & & & & Untergrenze & Obergrenze \\
\hline \multirow[t]{3}{*}{1} & 2 & ,072 & ,142 & 1,000 &,- 310 & ,455 \\
\hline & 3 &,- 441 & , 165 & ,052 &,- 885 & ,003 \\
\hline & 4 &,$- 910^{*}$ & , 137 & ,000 & $-1,278$ &,- 542 \\
\hline \multirow[t]{3}{*}{2} & 1 &,- 072 & , 142 & 1,000 &,- 455 & ,310 \\
\hline & 3 &,$- 514^{*}$ & , 181 & ,033 & $-1,001$ &,- 026 \\
\hline & 4 &,$- 982^{*}$ & ,173 & ,000 & $-1,446$ &,- 518 \\
\hline \multirow[t]{3}{*}{3} & 1 & ,441 & , 165 & 052 &,- 003 & ,885 \\
\hline & 2 &, $514^{*}$ & , 181 & ,033 & ,026 & 1,001 \\
\hline & 4 &,- 468 &, 175 &, 052 &,- 940 & ,003 \\
\hline \multirow[t]{3}{*}{4} & 1 & ,910* & , 137 & ,000 & ,542 & 1,278 \\
\hline & 2 & ,982* &, 173 & ,000 & ,518 & 1,446 \\
\hline & 3 & ,468 &, 175 & ,052 &,- 003 & ,940 \\
\hline
\end{tabular}

Basierend auf geschätzten Randmitteln

*. die Mittelwertdifferenz ist auf der Stufe ,05 signifikant.

b. Anpassung für Mehrfachvergleiche: Bonferroni. 
Tests der Innersubjekteffekte

Maß: MEASURE_1

\begin{tabular}{|c|c|c|c|c|c|c|c|c|}
\hline Quelle & $\begin{array}{l}\text { Typ III } \\
\text { Quadrat- } \\
\text { summe }\end{array}$ & df & $\begin{array}{l}\text { Quadrati- } \\
\text { scher Mit- } \\
\text { telwert }\end{array}$ & $\mathrm{F}$ & Sig. & $\begin{array}{l}\text { Partielles } \\
\text { Eta hoch } \\
\text { zwei }\end{array}$ & $\begin{array}{l}\text { De- } \\
\text { zentr. } \\
\text { Para- } \\
\text { meter }\end{array}$ & $\begin{array}{l}\text { Beobach- } \\
\text { tete } \\
\text { Trenn- } \\
\text { schärfe }^{a}\end{array}$ \\
\hline $\begin{array}{l}\text { Angenommene } \\
\text { Sphärizität }\end{array}$ & 68,694 & 3 & 22,898 & 15,490 &, 000 &, 123 & 46,471 & 1,000 \\
\hline Greenhouse-Geisser & 68,694 & 2,765 & 24,841 & 15,490 &, 000 &, 123 & 42,836 & 1,000 \\
\hline Huynh-Feldt (HF) & 68,694 & 2,844 & 24,156 & 15,490 &, 000 &, 123 & 44,052 & 1,000 \\
\hline Untergrenze & 68,694 & 1,000 & 68,694 & 15,490 &, 000 &, 123 & 15,490 & ,974 \\
\hline
\end{tabular}

a. Berechnet mit alpha $=, 05$

Deskriptive Statistiken

\begin{tabular}{|l|l|l|l|}
\hline & Mittelwert & $\begin{array}{l}\text { Standardabwei- } \\
\text { chung }\end{array}$ & H \\
\hline Planung_Problem_Summe & 1,6847 & 1,45840 & 111 \\
AnAbreise_Problem_Summe & 1,6126 & 1,66881 & 111 \\
Unterkunft_Problem_Summe & 2,1261 & 1,71685 & 111 \\
Freizeitangebot_Prob- & 2,5946 & 1,81598 & 111 \\
lem_Summe & & & \\
\hline
\end{tabular}


Tabellen zur statistischen Analyse von Hypothese 2: Problemempfinden

Mauchly -Test aus Sphärizität

\begin{tabular}{|l|l|l|l|l|l|l|l|}
\hline & & & & & \multicolumn{3}{|c|}{ Epsilon } \\
\hline $\begin{array}{l}\text { Innersubjekt- } \\
\text { effekt }\end{array}$ & $\begin{array}{l}\text { Mauchly } \\
\text {-W }\end{array}$ & $\begin{array}{l}\text { Approx. } \\
\text { Chi- } \\
\text { Quadrat }\end{array}$ & df & Sig. & $\begin{array}{l}\text { Green- } \\
\text { house- } \\
\text { Geisser }\end{array}$ & $\begin{array}{l}\text { Huynh } \\
\text {-Feldt }\end{array}$ & $\begin{array}{l}\text { Unter- } \\
\text { grenz } \\
\text { e }\end{array}$ \\
\hline $\begin{array}{l}\text { Problememp- } \\
\text { finden }\end{array}$ & 0,864 & 15,848 & 5 & 0,007 & 0,916 & 0,942 & 0,333 \\
\hline
\end{tabular}

Paarweise Vergleiche

\begin{tabular}{|c|c|c|c|c|c|c|}
\hline \multirow[t]{2}{*}{$\begin{array}{l}\text { (I)Prob- } \\
\text { lememp- } \\
\text { finden }\end{array}$} & \multirow[t]{2}{*}{$\begin{array}{l}\text { (J)Prob- } \\
\text { lememp- } \\
\text { finden }\end{array}$} & \multirow[t]{2}{*}{$\begin{array}{l}\text { Mittlere Dif- } \\
\text { ferenz (I-J) }\end{array}$} & \multirow[t]{2}{*}{$\begin{array}{l}\text { Stan- } \\
\text { dard } \\
\text { Fehler }\end{array}$} & \multirow[t]{2}{*}{ Sig.b } & \multicolumn{2}{|c|}{$\begin{array}{l}\text { 95\% Konfidenzintervall für die } \\
\text { Differenzb }\end{array}$} \\
\hline & & & & & Untergrenze & Obergrenze \\
\hline 1 & 2 &, $332 *$ & 0,107 & 0,015 & 0,044 & 0,619 \\
\hline & 3 & $-0,277$ & 0,121 & 0,143 & $-0,603$ & 0,048 \\
\hline & 4 &,$- 501 *$ & 0,094 & 0,00 & $-0,752$ & $-0,249$ \\
\hline 2 & 1 &,$- 332 *$ & 0,107 & 0,015 & $-0,619$ & $-0,044$ \\
\hline & 3 &,$- 609 *$ & 0,132 & 0,00 & $-0,962$ & $-0,256$ \\
\hline & 4 &,$- 832 *$ & 0,116 & 0,00 & $-1,145$ & $-0,52$ \\
\hline 3 & 1 & 0,277 & 0,121 & 0,143 & $-0,048$ & 0,603 \\
\hline & 2 &, $609 *$ & 0,132 & 0,00 & 0,256 & 0,962 \\
\hline & 4 & $-0,223$ & 0,112 & 0,292 & $-0,525$ & 0,078 \\
\hline 4 & 1 &, $501 *$ & 0,094 & 0,00 & 0,249 & 0,752 \\
\hline & 2 &, $832 *$ & 0,116 & 0,00 & 0,52 & 1,145 \\
\hline & 3 & 0,223 & 0,112 & 0,292 & $-0,078$ & 0,525 \\
\hline
\end{tabular}

Basiert auf den geschätzten Rand-

mitteln

* Die mittlere Differenz ist auf dem ,05-Niveau signifi-

kant.

b Anpassung für Mehrfachvergleiche: Bonfer-

roni. 
Test der Innersubjektiveffekte

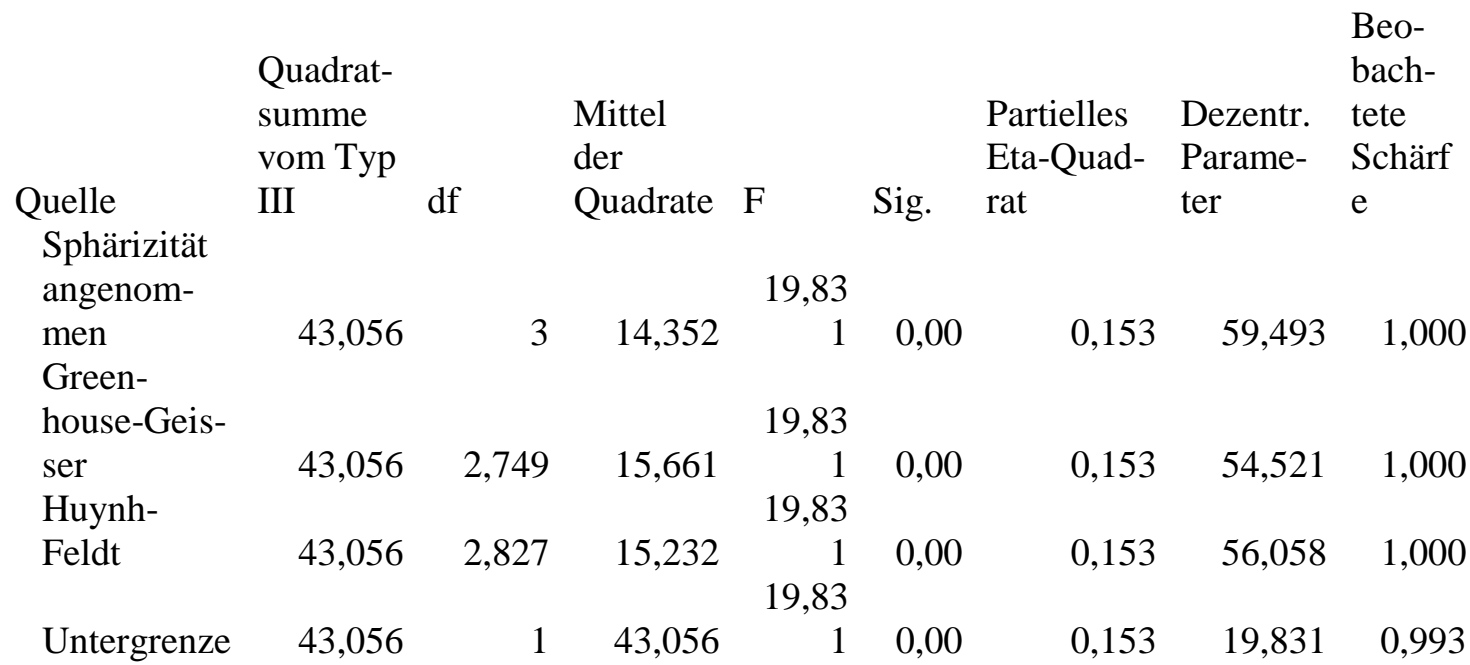

Deskriptive Statistiken

\begin{tabular}{|c|c|c|c|}
\hline & Mittelwert & $\begin{array}{l}\text { Std.-Abwei- } \\
\text { chung }\end{array}$ & $\mathrm{N}$ \\
\hline Planung_Mittelwert_Störfaktor & 1,0919 & 1,09205 & \\
\hline $\begin{array}{l}\text { AbAbreise_Mittelwert_Störfak- } \\
\text { tor }\end{array}$ & 0,7604 & 1,22543 & \\
\hline $\begin{array}{l}\text { Unterkunft_Mittelwert_Störfak- } \\
\text { tor }\end{array}$ & 1,3694 & 1,30096 & \\
\hline Freizeit_Mittelwert_Störfaktor & 1,5928 & 1,30563 & \\
\hline
\end{tabular}




\section{Tabellen zur statistischen Analyse von Hypothese 3}

\section{Mauchly-Test auf Sphärizität ${ }^{\mathrm{a}}$}

Maß: MEASURE_1

\begin{tabular}{|c|c|c|c|c|c|c|c|}
\hline \multirow[b]{2}{*}{$\begin{array}{l}\text { Innersubjektef- } \\
\text { fekt }\end{array}$} & \multirow[b]{2}{*}{$\begin{array}{l}\text { Mauchly- } \\
\text { W }\end{array}$} & \multirow[b]{2}{*}{$\begin{array}{l}\text { Näherungs- } \\
\text { weise Chi- } \\
\text { Quadrat }\end{array}$} & \multirow[b]{2}{*}{$\mathrm{df}$} & \multirow[b]{2}{*}{ Sig. } & \multicolumn{3}{|l|}{ Epsilon $^{\mathrm{b}}$} \\
\hline & & & & & $\begin{array}{l}\text { Greenhouse- } \\
\text { Geisser }\end{array}$ & $\begin{array}{l}\text { Huynh-Feldt } \\
\text { (HF) }\end{array}$ & $\begin{array}{l}\text { Unter- } \\
\text { grenze }\end{array}$ \\
\hline Faktor1 & ,920 & 9,048 & 5 & , 107 & ,950 & ,977 &, 333 \\
\hline
\end{tabular}

Testet die Nullhypothese, dass die Fehlerkovarianzmatrix der orthonormalisierten transformierten abhängigen Variablen proportional zu einer Identitätsmatrix ist.

\section{a. Design: Konstanter Term}

Innersubjektdesign: Faktor1

b. Kann für die Anpassung der Freiheitsgrade für die gemittelten Tests auf Signifikanz verwendet werden.

Korrigierte Tests werden in der Tabelle 'Tests der Innersubjekteffekte' angezeigt.

\section{Paarweise Vergleiche}

Maß: MEASURE_1

\begin{tabular}{|c|c|c|c|c|c|c|}
\hline \multirow[b]{2}{*}{ (I) Faktor1 } & \multirow[b]{2}{*}{ (J) Faktor1 } & \multirow{2}{*}{$\begin{array}{l}\text { Mittelwertdiffe- } \\
\text { renz (I-J) }\end{array}$} & \multirow[b]{2}{*}{ Standardfehler } & \multirow[b]{2}{*}{ Sig. ${ }^{b}$} & \multicolumn{2}{|c|}{$\begin{array}{l}95 \% \text { Konfidenzintervall für Dif- } \\
\text { ferenz }{ }^{\mathrm{b}}\end{array}$} \\
\hline & & & & & Untergrenze & Obergrenze \\
\hline \multirow[t]{3}{*}{1} & 2 &,- 171 & ,077 &, 165 &,- 377 & ,035 \\
\hline & 3 &, 045 & ,064 & 1,000 &,- 128 & ,218 \\
\hline & 4 &,- 153 & ,081 & ,370 &,- 371 & ,065 \\
\hline \multirow[t]{3}{*}{2} & 1 &, 171 & ,077 &, 165 &,- 035 & 377 \\
\hline & 3 &, $216^{*}$ & ,068 & ,012 & ,033 & ,399 \\
\hline & 4 &, 018 & ,075 & 1,000 &,- 182 & ,218 \\
\hline \multirow[t]{3}{*}{3} & 1 &,- 045 &, 064 & 1,000 &,- 218 & ,128 \\
\hline & 2 &,$- 216^{*}$ & ,068 &, 012 &,- 399 &,- 033 \\
\hline & 4 &,- 198 &, 074 &, 053 &,- 398 & ,002 \\
\hline \multirow[t]{3}{*}{4} & 1 &, 153 &, 081 & ,370 &,- 065 & ,371 \\
\hline & 2 &,- 018 &, 075 & 1,000 &,- 218 &, 182 \\
\hline & 3 & ,198 &, 074 & ,053 &,- 002 & ,398 \\
\hline
\end{tabular}

Basierend auf geschätzten Randmitteln

*. die Mittelwertdifferenz ist auf der Stufe ,05 signifikant.

b. Anpassung für Mehrfachvergleiche: Bonferroni. 
Tests der Innersubjekteffekte

Maß: MEASURE_1

\begin{tabular}{|ll|l|l|l|l|l|l|}
\hline Quelle & $\begin{array}{l}\text { Typ III } \\
\text { Quadrat- } \\
\text { summe }\end{array}$ & df & $\begin{array}{l}\text { Quadrati- } \\
\text { scher Mit- } \\
\text { telwert }\end{array}$ & F & Sig. & $\begin{array}{l}\text { Partielles } \\
\text { Eta hoch } \\
\text { zwei }\end{array}$ \\
\hline Faktor1 & $\begin{array}{l}\text { Angenommene } \\
\text { Sphärizität }\end{array}$ & 3,917 & 3 & 1,306 & 4,359 &, 005 &, 038 \\
& Greenhouse-Geisser \\
& 3,917 & 2,849 & 1,375 & 4,359 &, 006 &, 038 \\
& Huynh-Feldt (HF) & 3,917 & 2,932 & 1,336 & 4,359 &, 005 &, 038 \\
& Untergrenze & 3,917 & 1,000 & 3,917 & 4,359 &, 039 &, 038 \\
\hline
\end{tabular}

\section{Deskriptive Statistiken}

\begin{tabular}{|l|l|l|l|}
\hline & Mittelwert & $\begin{array}{l}\text { Standardabwei- } \\
\text { chung }\end{array}$ & $\mathrm{H}$ \\
\hline $\begin{array}{l}\text { Information28Planung_Skala } \\
\text { Information33Anreise_Skala } \\
\text { Information31Unter- }\end{array}$ & 1,49 &, 796 & 111 \\
kunft_Skala & 1,44 &, 889 & 111 \\
$\begin{array}{l}\text { Information35Ausflugs- } \\
\text { ziele_Skala }\end{array}$ & 1,64 &, 818 & 111 \\
\hline
\end{tabular}

\section{Tabellen zur statistischen Analyse von Hypothese 4}

\begin{tabular}{|l|r|r|r|r|r|}
\hline & & & Deskriptive Statistiken & \\
& $\mathrm{H}$ & Mittelwert & $\begin{array}{r}\text { Standardab- } \\
\text { weichung }\end{array}$ & Minimum & Maximum \\
\hline Buchung_Code & 111 & 1,9099 & 1,39837 & 1,00 & 6,00 \\
\hline
\end{tabular}

\section{Buchung:}

1 Privat

2 Privat und Reisebüro

3 Reisebüro

4 Freunde und Familie

5 Spezialreisebüros/- Anbieter

6 Rollstuhl/Behindertenverein 


\begin{tabular}{|l|r|r|r|}
\hline & $\begin{array}{c}\text { Buchung_Code } \\
\text { Anzahl }\end{array}$ & $\begin{array}{c}\text { Erwartete } \\
\text { Anzahl }\end{array}$ & Residuum \\
\hline 1,00 & 64 & 18,5 & 45,5 \\
2,00 & 21 & 18,5 & 2,5 \\
3,00 & 13 & 18,5 & $-5,5$ \\
4,00 & 4 & 18,5 & $-14,5$ \\
5,00 & 3 & 18,5 & $-15,5$ \\
6,00 & 6 & 18,5 & $-12,5$ \\
Gesamtsumme & 111 & & \\
\hline
\end{tabular}

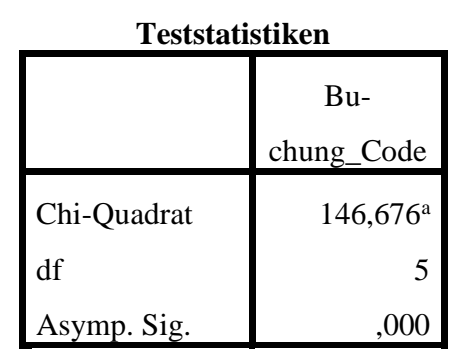

a. 0 Zellen $(, 0 \%)$ haben erwartete Häufigkeiten, die kleiner als 5 sind. Die kleinste erwartete Zellenhäufigkeit ist 18,5 .

Tabellen zur statistischen Analyse von Hypothese 5

\section{Chi Quadrat Test: Informationen zur Urlaubsplanung}

\section{Buchung_Info_Code}

\begin{tabular}{ll|l|l} 
& Beobachtetes N & Erwartete Anzahl & Residuum \\
\hline 1,00 & 23 & 12,3 & 10,7 \\
\hline 2,00 & 4 & 12,3 & $-8,3$ \\
\hline 3,00 & 40 & 12,3 & 27,7 \\
\hline 4,00 & 1 & 12,3 & $-11,3$ \\
\hline 5,00 & 4 & 12,3 & $-8,3$ \\
\hline 7,00 & 7 & 12,3 & $-5,3$ \\
\hline 8,00 & 17 & 12,3 & 4,7 \\
\hline 9,00 & 3 & 12,3 & $-9,3$ \\
\hline 10,00 & 12 & 12,3 &,- 3 \\
\hline Gesamt & 111 & & \\
\hline
\end{tabular}




\section{Statistik für Test}

\begin{tabular}{ll} 
& $\begin{array}{l}\text { Bu- } \\
\text { chung_Info_Code }\end{array}$ \\
\hline Chi-Quadrat & $104,108^{\text {a }}$ \\
\hline df & 8 \\
\hline Asymptotische Signifikanz &, 000 \\
\hline $\begin{array}{l}\text { a. Bei 0 Zellen (0,0\%) werden weniger als 5 Häufig- } \\
\text { keiten erwartet. Die kleinste erwartete Zellenhäufig- } \\
\text { keit ist 12,3. }\end{array}$
\end{tabular}

\section{Chi Quadrat Test: Reiseassisten und Geschlecht, Hypothese 6}

\section{Verarbeitete Fälle}

\begin{tabular}{|c|c|c|c|c|c|c|}
\hline & \multicolumn{6}{|c|}{ Fälle } \\
\hline & \multicolumn{2}{|c|}{ Gültig } & \multicolumn{2}{|c|}{ Fehlend } & \multicolumn{2}{|c|}{ Gesamt } \\
\hline & $\mathrm{N}$ & Prozent & $\mathrm{N}$ & Prozent & $\mathrm{N}$ & Prozent \\
\hline $\begin{array}{l}\text { Geschlechtergruppe * Reiseas- } \\
\text { sistenz }\end{array}$ & 107 & $96,4 \%$ & 4 & $3,6 \%$ & 111 & $100,0 \%$ \\
\hline
\end{tabular}

\section{Geschlechtergruppe * Reiseassistenz Kreuztabelle}

\begin{tabular}{|c|c|c|c|c|c|}
\hline & & & Reise: & & \\
\hline & & & Nein & $\mathrm{Ja}$ & Gesamt \\
\hline Geschlechtergruppe &, 00 & Anzahl & 48 & 13 & 61 \\
\hline & & Erwartete Anzahl & 46,2 & 14,8 & 61,0 \\
\hline & 1,00 & Anzahl & 33 & 13 & 46 \\
\hline & & Erwartete Anzahl & 34,8 & 11,2 & 46,0 \\
\hline Gesamt & & Anzahl & 81 & 26 & 107 \\
\hline & & Erwartete Anzahl & 81,0 & 26,0 & 107,0 \\
\hline
\end{tabular}

\section{Chi-Quadrat-Tests}

\begin{tabular}{ll|l|l|l|l} 
& & & $\begin{array}{l}\text { Asymptotische } \\
\text { Signifikanz } \\
\text { (zweiseitig) }\end{array}$ & $\begin{array}{l}\text { Exakte Signifi- } \\
\text { kanz (2-seitig) }\end{array}$ & $\begin{array}{l}\text { Exakte Sig- } \\
\text { nifikanz (1- } \\
\text { seitig) }\end{array}$ \\
\hline Chi-Quadrat nach Pearson &, $689^{\mathrm{a}}$ & 1 &, 407 & & \\
\hline
\end{tabular}




\begin{tabular}{ll|l|l|l|l|l}
\hline Kontinuitätskorrektur & , 363 & 1 &, 547 & & \\
\hline Likelihood-Quotient &, 684 & 1 &, 408 & & \\
\hline Exakter Test nach Fisher & & & &, 496 &, 273 \\
\hline Anzahl der gültigen Fälle & 107 & & & & \\
\hline
\end{tabular}

a. 0 Zellen $(0,0 \%)$ haben eine erwartete Häufigkeit kleiner 5. Die minimale erwartete Häufigkeit ist 11,18.

b. Wird nur für eine $2 \times 2$-Tabelle berechnet

\section{Weitere Ergebnisse}

Wie kann Reiseassistenz realisiert werden.

\section{Reiseassistenz_Traeger}

\begin{tabular}{|c|c|c|c|c|c|}
\hline & & Häufigkeit & Prozent & Gültige Prozente & $\begin{array}{l}\text { Kumulierte Pro- } \\
\text { zente }\end{array}$ \\
\hline \multirow[t]{6}{*}{ Gültig } & & 83 & 74,8 & 74,8 & 74,8 \\
\hline & Persönliches Budge & 9 & 8,1 & 8,1 & 82,9 \\
\hline & Privat & 9 & 8,1 & 8,1 & 91,0 \\
\hline & Sonstiges & 1 & 9 & 9 & 91,9 \\
\hline & Verein & 9 & 8,1 & 8,1 & 100,0 \\
\hline & Gesamt & 111 & 100,0 & 100,0 & \\
\hline
\end{tabular}

